九州大学学術情報リポジトリ

Kyushu University Institutional Repository

\title{
An Enumeration Of Micronesian Plants
}

Kanehira, Ryozo

Department of Agriculture, Kyusyu Imperial University

https://doi.org/10.5109/22573

出版情報 : 九州大学大学院農学研究院紀要. 4 (6)，pp.237-435，1935-11. Kyushu Imperial University バージョン：

権利関係 : 
Journal of the Department of Agriculture, Kyushu Imperial University, Vol. 4, No. 6. November 30, 1935.

\title{
AN ENUMERATION OF MICRONESIAN PLANTS
}

\author{
Ryôzô KANEHIRA
}

Micronesia, Japanese Mandate Territory, extending from Longitude $130^{\circ} \mathrm{E}$ to $175^{\circ}$ and from the Equator to $22^{\circ} \mathrm{N}$. Latitude, includes more than 1400 islands, islets and reefs comprising the Marianne, Caroline and Marshall groups. The total land area is but 2149 sq. $\mathrm{km}$. Except for the Marshall Group of atolls most of the islands are of volcanic origin with a covering of coral rock. They lie far to the west of Hawaii, east of the Philippines and Celebes, north of the New Guinea and Bismarck Islands and south of the Bonins and the Iwô Islands. The name of the principal islands, their areas and highest elevations are as follows:

\begin{tabular}{|c|c|c|c|c|c|}
\hline \multicolumn{4}{|c|}{ Marianne group } & \multicolumn{2}{|c|}{ West Caroline group } \\
\hline $\begin{array}{l}\text { Name of } \\
\text { island }\end{array}$ & $\begin{array}{c}\text { Area } \\
\text { (sq. km.) }\end{array}$ & $\begin{array}{c}\text { Highest } \\
\text { elevation }(\mathrm{m})\end{array}$ & $\begin{array}{l}\text { Name of } \\
\text { island }\end{array}$ & $\begin{array}{c}\text { Area } \\
(\mathrm{sq} . \mathrm{km} \text {.) }\end{array}$ & $\begin{array}{l}\text { Highest } \\
\text { elevation (m) }\end{array}$ \\
\hline Saipan & 185 & 474 & Yap & 216 & 179 \\
\hline Tinian & 98 & 172 & Palau & 378 & 206 \\
\hline Rota & 125 & 492 & Truk & 132 & 410 \\
\hline
\end{tabular}

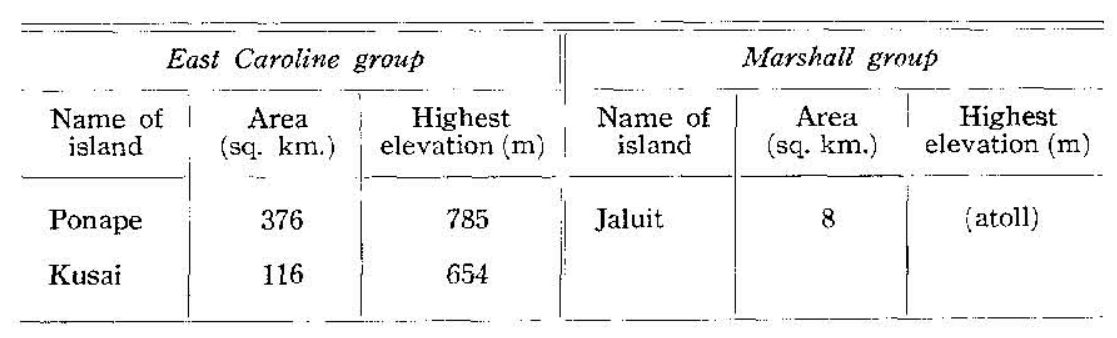

The first botanical collections made in this region were those by Thapdaeus HaEnke and LuIs NÉE, botanists of the Malaspina 
Expedition which reached Guam February 12th, 1792. HAENKE's material was studied by K. B. PRESL ${ }^{13}$ and some of NÉE's specimens were studied by CAVANILLES ${ }^{2}$. Following this expedition ADELBERT VON CHAMISSO and JOHAN FRIEDRICH ESCHSCHOLTZ reached Guam in November 24th, 1817 on the Russian brig Rurik (the Romanzoff Expedition). They spent some days in Radak, Marshall Islands, in January, the same year. The most successiful early collector was CHARLES GAUdiChaud-BEAup'sF, a French botanist, who reached Guam March 17th, 1819 on the "Uranie" commanded by Luis DE FReYcinet. He botanized Guam, Tinian and Rota. Besides the above mentioned botanists, several others visited the islands during the Spanish, régime, their work being described in SAFFORD's " Useful Plants of Guam."

Previous the time when the Marshall Islands were under the control of Germany, they were visited in 1880 by BeTCHF $^{6)}$ who published a short report and later when Micronesia was under German control, G. VoLKENS explored Yap in July 1899, staying some days also in the Marshall and Palau islands. He remained in Yap about six months, and later published several papers ${ }^{i}$, among

1) PRlsl, K. B., Reliquiae Hankeanae, seu descriptiones et icones plantarum, quas in America meridionali et boreali, in insulis, Philippinis et Marianis collegit Thaddeus Haenke Vol. 1, 2 (1825-36).

2) CaVANuldis A. J., Icones descriptiones plantarum, quae aut sponte in hispania crescunt aut in hortis hospitantur 1 (1791) $1-67$, t. $1-100 ; 4$ (1799) 1-82, t. $301-400 ; 5(1799) 1-74$, t. $401-500$.

3) Citamisso, A. von, et Schlichtrandal, D. von: De plantes expeditione speculatoria Romanzoffana observatis rationem discunt: Linnaea 1-10 (1826-1836).

4) Gindchaur-penurki, C., Botanique du voyage autour du monde fait par ordre du Roi sur les corvettes l' Uranie et la Physicienne pendant les années 1817-20 par M. Luis de Freycinet (1826-30).

5) Contribution from the U.S. National Herbarium Vol. 10 (1905).

6) Beтеrf, E., Vegetationsskizzeder Marshalls-Inseln - Wittmack's Gartenzcitung 3 (1884) 133-134.

7) Vorkl:Ns, G., Ueber die Karoninen-Insel Yap: Verhand. Gesell. Erdk. Berlin (1901) 62-76, Pl. 1.

-_ Die Vegetation der Karolinen, mit besonderer Berücksichtung der von Yap: Eng:. Bot. Jahrh. 31 (1901) 412-447.

ENGL. 1. c. $459-477$.

- L Einige Ergebnisse einer Reise nach den Karolinen u. Mariannen : Verhand. des XIII Deutsch. Geographentages Breslau (1901) 167-179.

—, Die Flora der Marshall-Inseln : Notizb. Bot. Gart. Berlin. 4 (1903) 83-91. 
which his "Aufzählung der auf Yap beobachteten und gesammelten Pflanzen" is the first enumeration of plants from that island. This comprises 328 species, distributed into 252 genera and 50 families; about 20 new species were described.

During the German régime, several collections were made by Jesuit Missionaries and by various Government officials stationed in the islands; the material was sent to the Botanical Garden at Berlin-Dahlem. H. HALLIER made some extensive collections in the Marianne Islands in 1902, his collections being preserved in Institut für allgemeine Botanik der Universität, Hamburg, but no list has been published. The most extensive collections were those by L. LEDERMANx who was a member of the Sepik Expedition to New Guinea. Separating from this group, he proceeded to Micronesia and botanized in Ponape and Palau from November 1913 to April 1914, securing about 1500 specimens. His collections were studied by various specialists at the Berlin Botanical Garden and the result appeared in several papers".

In the early part of the present century the island of Guam, an American possesion was explored and SAFFORD published his rather exhaustive work above mentioned in 1905. The first scientific enumeration was that by E. D. MERRILL", "An enumeration of the plants of Guam " and "Additions to the flora of Guam." The enumeration comprises 555 species of pterophytes and sper. matophytes.

In 1914-1915, while Micronesia was under the control of the Japanese Navy, the Department of Education sent several staff members of various Colleges and Universities to the region and some botanical material was secured by them. Among them was G. KoIDzumi ${ }^{3)}$ who published a number of new species based on material collected by him.

After 1915 no collections of botanical material were made in

1) Vurzens, G., Beiträge zur Flora von Micronesien (1) in FNGI.. Bot. Jahrb. 52 (1915) 1-18. DifL.s, L., Beiträge zur Flora von Micronesien und Polynesiens (2) ibicl. 56 (1921) 429-577, (3) ibid. 59 (1925) 1-29; (4) ibicl. 63 (1930) 292-324.

2) M:kkill, E. D., An enumeration of the plants of Guam i: Philip. Journ. Sci. 9 (1914) Bot. 14-155.

- - Addition to the flora of Guam. ibid. I5 (1919) 53344. $242 \cdot 257$

3) K rnzesi, G., The vegetation of Jaluit Island: Bot. Mag. Tokyo 29 (1915)

-_- Plantae novae micronesiae: ibicl. 30 (1916) 400-403, 31 1917) 232-233. 
the islands until 1929. The author botanized in Palau and Ponape in 1929, Saipan and Yap in 1930, Kusai and Ponape in 1931, Rota, Tinian and Palau in 1932 and in the northern islands of the Marianne group and Palau in 1933. The results were published in The Batanical Magazine, Tokyo and Transactions of the Natural History Society of Formosa $a^{1}$. He also prepared and published his "Flora Micronesica" providing a general sketch of the botanical status of Micronesia, describing the principal trees and shrubs and listing all plants known from the region.

In 1933-1934, T. HosoKawA ${ }^{2)}$ of the Taihoku Imperial University botanized in the islands and published several papers based on his own collections.

The present work is an emumeration of all plants known from Micronesia, not only from Japanese Mandate Territory but including also those of Guam, for Guam is geographically a part of the region. It thus serves as a general compilation covering the entire Micronesian flora. It includes 1219 species of plants representing 142 families and 616 genera, of which 456 species and eight genera are endemic in the region, while the manifestly introduced

1) Knмнцка, R., An enumeration of the woody plants collected in Micronesia, Japanese Mandate (1) Bot. Mag. Tokyo 45 (1931) 271-296, (2) 327-352.

-- New or noteworthy trees from Micronesia, (1) ibid. 56 (1922) 449-457, (2) $485-495$, 3) 669-674, (4) 47 (1933) 669-680, (5) 48 (1934) 116-130, (6) 400-405, (7) 830$736,(8) 919-927,99) 49$ (1935) 60-68, (10) 103-114, (11) 185-195, (12) $271-279$, (13) 352. 358, (14) $425-431$.

$1-8$.

_-..., Plantae novae micronesicae: Trans. Nat. IIist. Soc. Formos. 24 (1935)

-..-, Flora Micronesica (Napanese), published by the South Seas Bureat1, Japanese Mandate Territory (1933).

2. IV sukawa T, Materials of the botanical research towards the flora Micronesia (1): Trans. Nat. Hist. Soc. Formos. 24 (1934) 197-205; (2) 414-415; (3) 17-39; (4) $117-128$.

--- Conspectus of the genus Lepinia: Bot. Mag. Tokyo $48(1934 ; 528-530$.

Balanophoraceae Micronesiae: Journ. Soc. Trop. Agr. 6 (1934) 572.

-..-, Phytogeographical relationship between the Bonin and the Marianne Islands laving stress upon the distributions of the families, genera and special species of their vernacular plants: ibid. 6 (1934) 201-209, 657-670.

-.., Preliminary account of the vegetation of the Marianne Islands group (Japanese text. with English résumé): Bul. Biog. Soc. Jap. 5 (1934) 124-172. 
species number $230^{1}$.

Duplicate sets of botanical specimens have been distributed to the following institutions both at home and abroad: Tokyo, Kyoto and Taihoku Imperial Universities, Tokyo Science Museum, South Seas Bureau, the New York, Berlin-Dahlem, and Kew Gardens, the Paris Museum, the Arnold Arboretum, the Bishop Museum and the Smithsonian Institution.

The botanical exploration of Micronesia has been accomplished through assistance granted by the South Seas Bureau. The author especiaily desires to express his most sincere thanks to Dr. E. D. MERRILL of the New York Botanical Garden, whose encouragement and unceasing assistance has enabled him to accomplish this work. Acknowledgment is also due to Prof. L. Diels, Dr. F. Markgraf, and Dr. K. Kralse of Berlin-Dahlem, Dr. A. Gulllaumin of Paris Museum, the late Dr. U. Martelli of Florence, Italy and Dr. E. B. CoPELAND of the Philippines who have assisted the author in the identification of certain critical species.

In the following table is given the inclusive numbers and the dates of collection for the botanical material on which this paper is essentially based. Unless the name of collectors mentioned, it is collected by the author.

\section{9}

1-47 Saipan, June 27, 1929.

48-66 Tinian, June 29, 1929.

67-74 Yap. July 2, 1929.

75.142 Korror, Palau, July 4, 1929.

143-203 Korror, Palau July 6, 1929.

204-213 Korror, Palau July 7, 1929.

214.233 Uninhabited coral island, Korror,

Palau, July 8, 1929.
234-255 Uninhabited coral island, Korror, Palau, July 9, 1929.

256-331 Aimiriik, Palau, July 10, 1929.

332-348 Korror, Palau, July 12, 1929.

349-377 Korror, Palau, July 13, 1929.

378-427 Marikyoku, Palau, July 17, 1929.

428.455 Marikyoku, Palau, July 18, 1929.

456.467 Korror, Palau, July 20, 1929.

468-482 Armatin, Palau, July 21, 1929.

1) For phytogeographical data appartaining to the Micronesian flora, the following papers should be consulted:

Kavaitra, R., On the ligneous flora of Micronesia, Japanese Mandate (Japanese text) Journ. Japan Forest. Soc. 13 (1931) 755.787.

, The forest trees of Micronesia, Japanese Mandate: Trop. Woods, No. 29 (1932) $1-6$.

- Flora Micronesica (1933) 14-24.

-_, On the flora of Micronesia (Japanese text with English résumé) : Bull. Biogeogr. Soc. Jap. 5 (1935) 233-262.

- On the distribution of Pandanus and geographic position of Micronesian species: ibid. (ined.) 
483-520 Aimiyon, Palau, July 22, 1929. 521-541 Kamset, Palau, July 23, 1929. 542-565 Garamiscan, Palau, July 24, 1929. 566-601 Angaur, Palau, July 29, 1929. 602-611 Truk, Aug. 2, 1929.

612-625 Truk, Aug. 4, 1929.

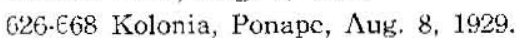
669-696 Kolonia, Ponape, Aug. 8, 1929. 697-743 Kolonia, Ponape, Aug. 10, 1929. 744-774 Sankakuyama, Ponape, Aug. 12, 1929.

775-791 Metaranium, Ponape, Aug. 13, 1929.

792-830 Niinoani-zan, Ponape, Aug. 14, 1929.

831-845 Niinoani-zan, Ponape, Agu. 15, 1929.

846-853 Metaranium, Ponape, Aug. 16, 1929.

864-882 Kolonia, Ponape, Aug. 19, 1929.

\section{0}

883-888 Saipan, July 22, 1930.

889-930 Saipan, July 23, 1929.

931.980 Saipan, July 25, 1930. 981-1001 Saipan, July 26, 1930. 1002-1927 Saipan, July 27, 1930. 1028-1039 Saipan, July 28, 1930 . 1040-1043 Saipan, July 30, 1930. 1044-1059 Tinian, July 30, 1930. 106()-1074 Tinian, July 31, 1930. 1075-1089 Saipan. Aug. 2, 1930. 1090-1136 Yap, Aug. 11, 1930. 1137-1172 Tomil, Yap, Aug. 12, 1930. 1173-1185 Tomil, Yap, Aug. 13, 1930. 1186-1211 Tomil. Yap, Aug. 14, 1930. 1212-1240 Maki, Yap, Aug. 15, 1930. 1241-125’2 Maki. Yap, Aug. 16, 1930. 1253-1263 Yap, Aug. 18, 1930. 1264 Yap, S. Iktira, Dec. 21, 1930. 1265 Ponape M. Hossis, Dec. 1930.

\section{1}

1266-1298 Tol, Truk, June 23, 24, 1931. 1299-1319 Lelo, Kusai June 30, 1931. 1320-1411 Hinkorn-zan, Kusai, July 2, 3, 1930 .

1412.1435 Moto, Kusai, July 6, 1931.
1436-1468 Matante, Kusai July, 7, 1931. 1469-1475 Kolonia, Ponape, July 11, 1931 1476-1491 Kolonia, Ponape, July 12, 1931 1492-1524 Parkier, Ponape, July 13, 1931. 1525-1549 Kity, Ponape July 16, 1931. 1550-1576 Tortom-zan, Ponape, July 18 1931.

1577-1600 Kolonia. Ponape, July 21, 1931. 1601-1622 Nânaraut-zan, Ponape July 23, 1931.

1623-1631 Nânaraut-zan, Ponape, July 24, 1931.

1632-1676 Nânaraut-zan, Ponape, July 25 1931.

1677-1685 Kolonia, Ponane, July 27. 1931 ' 1686-1691 Jokaji. Ponape, Aug. 1, 1931.

1692-1699 Metaranium, Ponape, Aug. 3: 1931.

1700-1717 Mocn, Truk, Aug. 14, 1931. 1718-1727 Fefan, Truk. Aug. 15. 1931. 1728 Palau Experiment St. Sept. 1931. 1729 Tinian Y. Irost, Sept. 1931.

1730 Tinian Y. Ikope, Sept. 1931.

\section{2}

1731-1773 Rota, July 8, 1932.

1774-1805 Rota. July 9, 1932.

1806-1822 Rota, July 10, 1932.

1823 Rota. July 11, 1932.

1846 Angaur, Palau, July 29, 1932.

1847-1853 Korror, Palau, Aug. 1, 1932.

1854-1888 Tôdai-yama, Palau, Aug. 2, 1932. 1889-1913 Uninhabited coral island, Korror,

Palau, Aug, 3, 1932.

1914-1953 Aimiriik, Palau, Aug. 5, 1932. 1954-1986 Aimiriik, Palau, Aug. 6, 1932. 1987-2019 Uninhabited coral island, Palau,

Aug. 8, 1932

2020-2029 Tôdai-yama, Palau, Aug. 9, 1932. 2030-2057 Marikyoku, Palau, Aug- 11, 1932. 2058-2066 Ogiwal, Palau, Aug. 13, 1932. 2067-2080 Korror, Palau, Aug. 15, 1932. 2081.2094 Arumonogui, Palau, Aug. 16 , 1932.

2095-2127 Garamiscan, Palau, Aug. 17, 1932.

2128-2131 Palau, S. Nisms Oct. 1932.

2132 Saipan, T. W $\triangle$ DA, Dec. 1932. 
2133 Palau, S. Nisirs, Oct. 1932.

\section{3}

2134 Ponape, M. Husno, Jan. 1. 1933. 2135 Palau Experiment Station, March, 1933.

2136 Saipan, T. Wada, March, 1933.

2137 Palau, S. Wnknmate; March, 1933.

2138 Ponape, M. Hosino, March 1933.

2139 Ponape, M. Histw, March 20, 1933. 2110 Ponape M. H:sino, Feb. 1933.

2141 Saipan, June 30, 1933.

2142-2154 Anatahan, July, 1933.

2155-2172 Saligan, July 5, 1933.

2173.2197 Aramagan, July, 1933.

2198-2202 Pagan, July, 1933.

2203-2209 Agrigan, July, 1933.

2210-2216 Pagan, July, 1933.

2217-2243 Saipan, July 11, 1933.

2241-2249 Saipan, July 12, 1933.

2250-2257 Saipan, K. Miti:DA, June, 1933.

2258-2278 Tinian, July 15, 1933.

2279-2304 Aimiriik, Palau, July 30, 1933. 2305-2336 Aimiriik, Palau, July 31, 1933. 2337-2359 Aimiriik, Palau, Aug. 1, 1933. 2360-2373 Aimiriik, Palau, Aug. 2, 1933. 2374-2381 Aimiriik to Airai, Palau, Aug. 4, 1933.

2382-2390 Airai, Palau, Aug. 5, 1933.

2391-2415, Uninhabited coral island, Arai,

Palat, Aug. 6, 1933.

2416-2425 Peliliu, Palau, Aug. 9, 1933.

2426-2441 Pelliu, Palau, Aug. 10, 1933.

2442-2478 Uninhabited coral island, Korror, Palau, Aug. 14, 1933.

2748 Yap, September 7, 1933.

2749-2752 Palau, S. NisiuA, March 8, 1933. 2753-2783 Palau, S. Nisiva, no date, 1933. 2784-2785 Saipan, K. Mituls, no date, 1933. 2786-2791 Jaluit, H. Utrman, Aug. 1933. 2792 Kusai, Yosida Aug. 1933.

2793-2795 Jaluii, Kavano, Aug. 1933.

2796 Kusti, H. Utrama, Aug. 1933. 2797-2803 Jaluit, H. UniYAma, Aug. 1938. 2804-2808 Kusai, H. Utryas, Aug., 1933. 2809-2812 Ponape, M. Hosrvo, no date, 1933.
2813-2815 Yap, Sept. 1933.

2816-2817 Truk, Simi\%, Aug. 1933.

2818 Palau, S. Nisida, Palau, Aug. 1933.

3340 Saipan, K. Mrtuds, Aug. 1933.

3341 Saipan, Aug. 1933.

3342 Palau, Aug. 1933.

3343-3345 Yap, Sept. 1933.

3346 Angaur, Palau, R. Ikrds, April, 1933. 3347-8 Korror, S. Nisids, April 12, 1933.

3352 Palau S. Nisida, Aug. 1933.

3353 Coral island, Korror, Palau, S. Nisuds, Aug. 1933.

\section{4}

3340 Korror, Palau, S. Nisida, Jan. 16, 1934. 3441 Aimiriik, Palau, S. Nisin, Feb. 27, 1934.

3442 Korror, Palau, S. Nisina, Feb. 28, 1934. 3443-4 Anathan, T. Wh1 , May, 1934. 3448-3456 Ponape, M. Hosin!, Aug. 1934. 3457-3466 Mokiel Oirs, Aug. 1933.

3467-3483 Enchabi OHBA, Aug. 1934.

3484 Wanietak, no name, 1934.

3485-3487 a nameless island, near Ponape, no name, 1933.

3488 Harusima, Truk K. KasuYa, Dec. 6. 1934.

3489 Tol, Truk K. Inf, Dec. 6, 1934.

3490 Natusima, Truk, Natu, Dec. 6, 1934,

3491 Tol, Truk, K. Isn, Dec. 6, 1934.

3492 Natusima, Truk, Naru⿰, Dec. 6, 1934.

3493 Huyusima, Truk, T. Yiпкимма, Dec. 6, 1934.

3494 Takesima, Truk, NA 3495 Akisima, Truk, T. Huкичмма, Dec. 6 , 1934.

\section{5}

3497-3502 Yap, Yap District Office, Jan. 17, 1935.

3503-3507 Kusai Received, Feb. 1935.

3508-3529 Jaluit Received, Feb. 13, 1935.

3530 Saipan, Aug. 1935.

3531 Truk, Dec. 1935.

3532 Rota, S. KLramoto, March 7, 1935. 


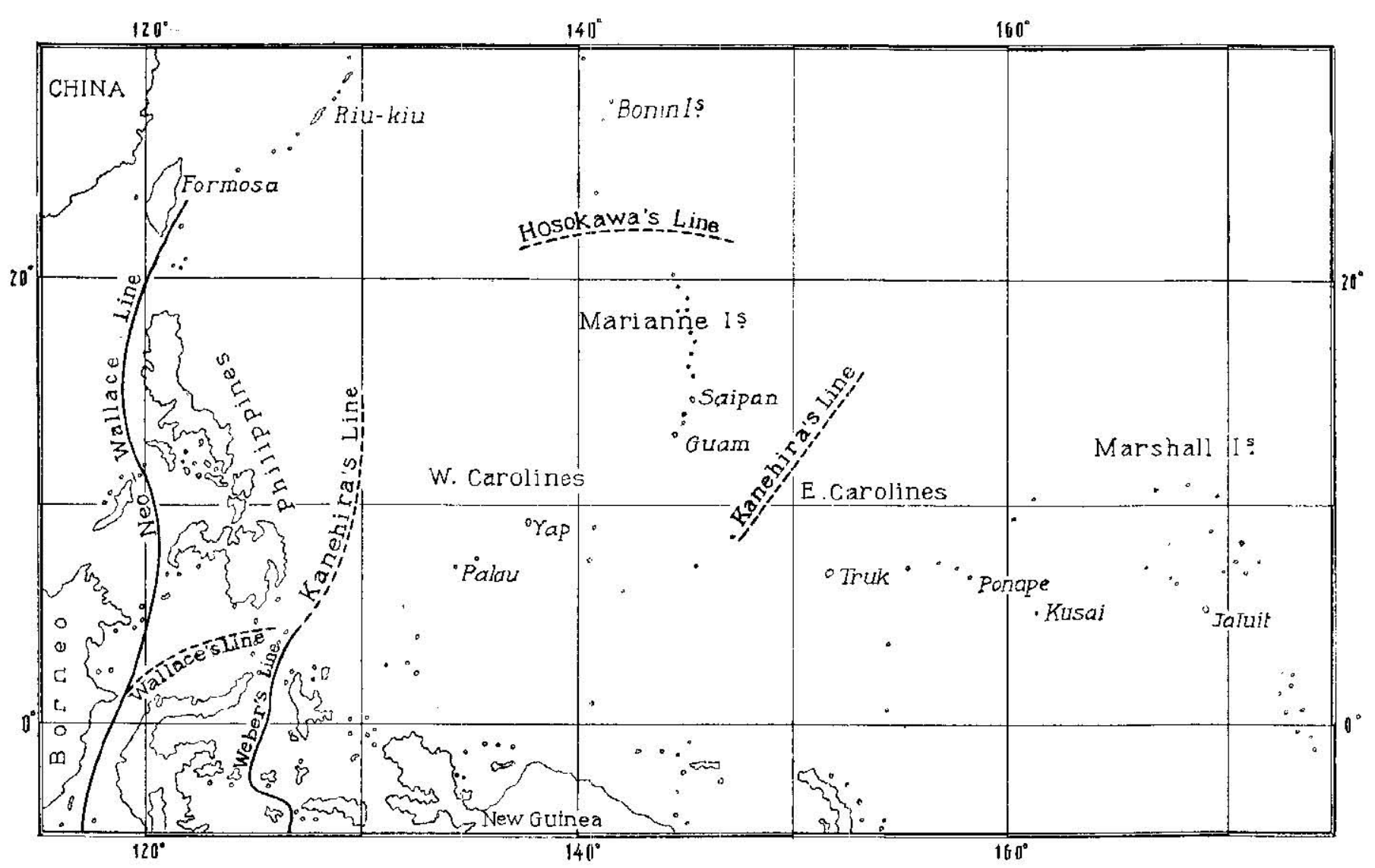

The Micronesian region, showing various lines of biogeographic demarcation 


\section{PTERIDOPHYTES}

\section{Marattiaceae}

\section{Angiopteris HoFFMANN}

Angiopteris evecta (Forst. f.) Hofrm. Comm. Soc. Reg. Gott. 12 (1796) 29, t. 5; VolKens in ENGL. Bot. Jahrb. 31 (1901) 454 ; Merr. in Philip. Journ. Sci. 9 (1914) Bot. 46.

Polypodium evectum Forst. f. Prodr. (1786) 81.

Palau, 550; Ponape 719, 725; Yap 1095; Kusai 1320, 1329; Anatahan 2142; Aramagan 2176. In damp primary forests at medium altitudes. Distrib. Polynesia.

\section{Marattia SwaRTz}

Marattia sambucina BL. Enum. (1828) 256.

Marattia fraxinea J. SM. Volkess 1. c. 454.

Ponape 759, 811 ; Kusai 1392 ; Palau 308. In damp primary forests.

Distrib. Malaysia.

\section{Ophioglossaceae}

\section{Helminthostachys KaUlfuss}

Helminthostachys zeylanica (LiNN.) Hook. Gen. Fil. P1. (1840) 47; VOLKENS 1. c. 454.

Yap (Volkens). Distrib. Tropical Asia to tropical Australia and New Caledonia.

\section{Ophioglossum LinaELS}

Ophioglossum pendulum LINN. Sp. P1. ed. 2 (1763) 1518; MERr. 1. c. 46 .

Palau 306 ; Kusai 1398, 1445. In forests at medium altitudes. Distrib. Tropical Asia to Polynesia.

Ophioglosum reticulatum LINN. Sp. Pl. ed. 2 (1763) 1063; VolKENS 1. c. 454 .

Yap (Volkens) Distrib. Tropical Africa, tropical America and Samoa.

Hymenophyllaceae

Hymenophyllum SмITH

Hymenophyllum Blumeanum Spr. Syst. 4 (1827) 131. 
Palau 495. Distrib. Tropical Asia. Jap. name: Taiwan-kokesinobu.

\section{Trichomanes LINNAEUS}

Trichomanes humile Forst. f. Prodr. (1786) 84; VolKens 1. c. 453; MERR. 1. c. 41.

Yap (Volkens), Guam (Merrill). Distrib. Hawaiian Islands to Polynesia, Formosa, New Zealand and Australia. Jap. name: Himehoragoke.

Trichomanes javanicum BL. Enum. (1828) 224 ; Vol.kens 1. c. 41. Ponape 757, 816; Yap 1124; Kusai 1408. In forests from low to medium altitudes. Distrib. Tropical Asia to the Riukiu Islands southward to Australia and Polynesia. Jap. name: Sotetu-horagoké.

Trichomanes maximum BL. Enum. (1828) 228.

Ponape 839, 1667. In forests at high altitudes. Distrib. Malaysia, Polynesia, Queensland. Jap, name: Seriba-horagoké.

Trichomanes pallidum BJ. Enum. (1828) 225.

Ponape 819. In forests at high altitudes. Distrib. Tropical Asia to Polynesia. Jap. name: Usuiro-kokesinobu.

Trichomanes rigidum Sw. Prodr. (1788) 137; VolKkNS 1. c. 435. Yap (Volkens). Distrit. Tropical Asia and Polynesia.

\section{Cyatheaceae}

\section{Alsophila R. BROWN}

Alsophila extensa (Forst. f.) R. Br. Prodr. (1810) 42 ; Merr. 1. c 41. Polypodium extensum Forst. f. Prodr. (1786) 83.

Guam (Merrils). Distrib. New Guinea, Celebes and Polynesia.

\section{Cyathea SMITH}

Cyathea affinis (FoRsT.) Sw. Schrad Journ. 1800-2 (1801) 94 ; Carr. in SeEm, Fl. Vit. (1873) 333; Kanehira in Bot. Mag. Tokyo 48 (1934) 730.

Polypodium affine Forst. Prodir. (1786) 83; BRACK Wilkes U.S. Expl. Exp. 16 (1854) 283.

Ponape 677, 810, 1493; Kusai 1345. In forests of Kusai and Ponape Islands. Distrib. Polynesia.

Cyathea Haenkei (Presl) Merr. in Philip. Journ. Sci. 15 (1919) 540. 
Alsophila Haenkei PResL Rel. Haenk. 1 (1825) 68.

Cyathea marianna GaUdicH. Bot. Freyc. Voy. (1826) 265.

Guam (MerriLl). "The type of PresL's species was from the Marianne Islands, in all probability from Guam, while that of GAUDICHALD was from Guam. The species is apparently very scarce and local in Guam and is probably approaching extinction." (MERRILL 1. c. 540).

Cyathea lunulata (Forster) CoPel,, Bishop Museum Bull. 59 (1929) 37 ; KANEHIRA 1. c. 730.

Polypodium lunulalum FonstER Prodr. (1786) 83.

Alsophila lumulata R. BR., HoOKER and BAKER Synopsis Fil. (1868) 41.

Palau: Korror 138, Aimiriik 263, 2291, Garamiscan 2100. Distrib. South-Western Polynesia and Melanesia.

Cyathea aramaganensis KANEHIRA in Bot. Mag. Tokyo 47 (1934) 731 .

Aramagan 2173 ! July 5, 1933. In primary forests at the top of the island. Distrib. Endemic.

Dicksonia L'HÉRITLER

Dicksonia cicutaris Sw. Schrad. Journ. Bot. (1801) 91; VolkENs 1. c. 453 .

Yap (Volkens). Distrib. Tropical America.

\section{Polypodiaceae}

\section{Acrosticum LINNAEUS}

Acrosticum aureum LINN. Sp. Pl. (1753) 106; VolkeNs 1. c. 454; MERR. 1. c. 42.

Palau 122; Yap 1190. Along the seashore. Distrib. Tropics of both hemispheres. Jap. name: Kogane-sida.

\section{Adianthum LINNAEUS}

Adiantum lunulatum Burm. Fl. Ind. (1768) 235; VolkEss 1. c. 453. Yap (Volkens). Distrib. Africa, Asia, Australia, Polynesia to C. America. 
Antrophyum plantagineum (CAV.) K^ULF. Enum. (1824) 197; VoLKENS 1. c. 453 ; MERR. 1. c. 42.

Hemionitis plantaginea CAv. Descr. (1802) 260.

Palau 251, 326, 383, 2332; Yap 1194. Distrib. Ceylon, Ilimalaya, Philippines to Polynesia. Jap. name: Ooba-kosida.

\section{Asplenium Linnaeus}

Asplenium adianthodides (LNN.) C. CHR. Index Fil. (1906) 99; MERR. 1. c. 42.

Trichomanes adianthoides LiNN. Sp. Pl. (1753) 99.

Kusai 1458, 1459; Palau 2112. In forests at high altitudes. Distrib. Troptcal Asia to Australia, New Zealand and Polynesia. Jap. name: Munin-sida.

Asplenium caudatum Forst. f. Prodr. (1876) 80; MerR. 1. c. 42. Saipan 25, 26 ; Ponape 676, 1613 ; Kusai 1455; Rota 1816. In forests at low altitudes. Distrib. Tropics of both hemispheres.

Asplenium laserpitiifolium LaM. Encycl. 2 (1786) 310; MERr. 1. c. 42.

Palau 498; Ponape 643, 806; Kusai 1397, 1440, 1448. In forests from low to high altitudes. Distrib. Malaya to Australia and Polynesia. Jap. name: Okinawa-sida.

Asplenium macrophyllum Sw. in Schrad. Journ. 1800-2 (1801) 52 ; MERR. 1. c. 42.

Saipan 25, 28, 29; Tinian 1067. In forests at low altitudes. Distrib. India to Mascarene and Polynesia. Jap. name: Ooba-muninsida.

Asplenium monanthes LINN. Mant. (1767) 130; MFrR, 1. c. 42. Guam (Merrill). Distrib. Tropical Africa and America to Hawaii.

Asplenium nidus Linn. Pl. (1753) 1079; Merr. 1. c. 42.

Palau 91; Ponape 713 ; Kusai 1395, 1457. In damp primary forests from low to high altitudes. Distrib. Himalaya, Japan, Formosa, eastwards to Society Islands, Jap.name: Ootani-watari.

Asplenium nitidum Sw. Syn. (1806) 84; MERr. 1. c. 42. Guam (MerRILL). Distrib. India to Malaya.

Asplenium pachyphyllum KTzE. Bot. Zeit. (1848) 146; HooK. Sp. Fill. 4 (1862) 56 ; VolkeNs 1. c. 453. 
Yap (Volkens). Distrib. Philippines, Malaya, Solomon, Fiji and Formosa. Jap. name:

Asplenium scandens J. Sm. in Ноок. Journ. Bot. 3 (1841) 408.

Ponape 627, 1622. In forests at high altitudes. Distrib. Philippines, New Guinea to Fiji.

Asplenium unilaterale LAM. Encyc. 2 (1786) 305.

Anatahan 2151. In primary forests. Distrib. Tropical Africa to Polynesia.

\section{Blechnum LiNNAFUS}

Blechnum orientale LinN. Sp. Pl. (1753) 1077; Volkens 1 c. 453; MERR. 1. c. 42.

Palau 133, 339, 2084 ; Ponape 731, 1516; Yap 1218; Kusai 1342. In thickets and forests at medium altitudes. Distrib. Tropical Asia to Polynesia. Jap. name: Hiryu-sida.

\section{Campium PREsi.}

Campium heteroclitum (Press) CoPel. in Philip. Journ. Sci. 37 (1928) Bot. 395, f. 13.

Acrosticum heteroclitum PRESL, Rel. Haenk. 1 (1825) 15, t. 2 , f. 2. Kusai 1340,1453 . in primary forests at medium altitudes. Distrib. India through Malaysia to the Philippines and New Guinea.

\section{Cheilanthes SWARTZ}

Cheilanthes tenuifolia (Burm. f.) Sw. Syn. (1806) 129, 332; VolKENS 1. c. 453 ; MerR. 1. c. 43.

Trichomanes tenuifolium BuRM. f. Fl. Ind. (1763) 237.

Palau 488; Yap 1223. In primary forests. Distrib. Tropical Asia to New Zealand and Polynesia. Jap. name: Sima-ebigarasida.

\section{Cyclopeltis Desvaux}

Cyclopeltis Presliana (J. SM.) Berkeley, Introd. Crypt. Bot. (1857) 517.

Lastrea Presliana J. Sm. nom. nud. (1841).

Palau 92, 1901. Distrib. Burma, Malaysia, Philippines to New Guinea. 


\section{Cyclophorus Desvaux}

Cyclophorus adnascens (Sw.) Dfsv. in Berl. Mag. 5 (1911) 300; MERR. 1. c. 43.

Polypodium adnascens Sw. Syn. (1806) 25, 222, t. 2, f. 2.

Palau 340, 467, 2015; Saipan 2242. In forests at low altitudes.

Distrib. Tropical Asia to Polynesia. Jap. name: Hituba-mamezuta.

\section{Davalia SmiTi}

Davalia elegans Sw. Hook. Sp. Fil. 1 (1846) 164.

Palau 224. Distrib. Ceylon, Malay Peninsula, China, Java, Australia and Polynesia.

Davalia solida (Forst. f.) Sw. in Schrad. Journ. 1800-2 (1801) 87; VOLKENS 1. c. 453 ; Merr. 1. c. 43.

Trichomanes solidum FoRst. f. Prodr. (1876) 86.

Palau 224, 2099; Truk 621; Ponape 703; Yap 1260. Distrib. Malaya to northern Australia and Polynesia. Jap. name: Atuba-sinobu.

\section{Dryopteris ADANSON}

Dryopteris adenophora C. CHR. Ind. Fil. (1905) 251.

Saipan 30 ; Palau 104, 1885; Ponape 730. In forests at low altitudes. Distrib. Luzon to Celebes.

Dryopteris cucullata (Bi..) ChкIST. in Philip. Journ. Sci. 2 (1907) Bot. 194 ; Merr. 1. c. 43.

Aspidium cucullatum Bu. Enum. (1828) 151.

Guam (Merrill). Distrib. Mascarene Islands and Malaya.

Dryopteris depauperata Cops.. in Philip. Journ. Sci. 9 (1914) Bot. 44:

Guam. Distrib. The type from Guam, endemic.

Dryopteris dissecta (Forst. f.) O. KTZF. Rev. Gen. Pl. 2 (1891) 812; MFRR. 1. c. 43.

Polypodium dissectum Forst. f. Prodr. (1786) 812.

Guam (MerriLl). Distrib. India to Madagascar, Malaya, Australia and Polynesia.

Dryopteris gongylodes (Sснкинк) O. KTzl: Rev. Gen. Pl. 2 (1891) 811 ; MERR. 1. c. 43. 
Aspidium gongylodes ScHKUHR Kr. Gew. 1 (1809) 193, t. 33.

Guam (Merrill). Distrib. Pantropic.

Dryopteris Haenkeana (PREsL) O. KTze. Rev. Gen. Pl. 2 (1891) 812; Merr. 1. c. 43.

Nephrodium Haenkeanum PRESL Epim. Bot. (1851) 46.

Guam (Merril.). Distrib. Malaya and Polynesia.

Dryopteris parasitica (LINN.) O. KTZE Rev. Gen. Pl. 2 (1891) 811; MERR. 1. c. 43.

Polypodium parasicum LinN. Sp. Pl. (1753) 1090.

Guam (MERRILL). Distrib. Warmer parts of both hemispheres. Jap. name: Ke-hosida.

Dryopteris simplicifolia (J. SM.) CHRIST. in Philip. Journ. Sci. 2 (1907) Bot. 206.

Nephorodium simplicifolia J. SM. (1841) nom.

Palau 533. Distrib. Philippines to Fiji.

Dryopteris sophoroides (ThunB.) O. KTZE. Rev. Gen. Pl. 2 (1891) 813.

Polypodium sophoroides Thuns. Tr. Linn. Soc. 2 (1794) 341.

Saipan 30, 2229; Kusai 1399. Distrib. India to China.

\section{Histiopteris J. Sм̄̄TH}

Histiopteris incisa (ThunB.) J. SM. Hist. Fil. (1875) 295.

Pteris incisa Tiunn. Prodr. Fl. Cap. (1800) 171.

Ponape 642, 1646. In forests from low to high altitudes. Distrib. In both hemispheres. Jap. names: Yuminonesida.

\section{Humata CAvANII.LFS}

Humata augusta (WAI.L.) J. Sm.

Palau, Arumonogui-zan 516. Distrib.

Humata gaimardiana (GAIdich.) J. SM. in Hook. Lond. Journ. Bot. 1 (1842) 425.

Nephrodium gaimardiana Gaudich. Freyc. Voy. Bot. (1827) 335, t. 12, f. 1.

Palau 225, 1931 ; Ponape 704. Distrib. Burma through Malaya and the Philippines to Polynesia.

Humata heterophylla (SM.) Drsv. Prodr. (1825) 323 ; MERr. 1. c. 44. 
Davalia heterophylla SM. in Mém. Acad. Trin. 5 (1783) 415.

Palau 515, 555. Distrib. Malaya and Polynesia.

Hymenolepis KaULFuss

Hymenolepis spicata (LINN. f.) PREsL Epim. (1851) 159; MERR. 1. c. 44 .

Acrostichum spicatum LinN. f. Suppl. (1761) 444.

Palau 497. Distrib. Tropical Asia, Madagascar, Malaya and Polynesia. Jap. name: Ogata-sida.

\section{Lindsaya DRYANDER}

Lindsaya decomposita WILld. Sp. 5 (1810) 425.

Palau 281, 2098; Ponape 755, 1648 ; Kusai 1346. Distrib. Tropical Asia, Australia and Polynesia.

Lindsaya Merrillii Copel. in Philip. Govern. Lab. Pub. 28 (1905) 61.

Ponape 808, 1614 ; Kusai 1437. Distrib. Philippines.

Lindsaya repens (BORY) BEDD. Ferns. S. Ind. (1863-5) 72, t. 209.

Dicksonia repens Bory Voy. 2 (1804) 323.

Palau 528. Distrib. Assam, Ceylon, Java, Sumatra, Polynesia and Mauritius. Jop. name: Hongu-sida.

\section{Microlepia PRESL}

Microlepia Speluncae (LINN.) T. MoORE Ind. Fil. 2 (1857) 93.

Ponape 644. Distrib. Pantropic. Jap. name: Oo-isikaguma.

\section{Nephrolepis ScHOTT}

Nephrolepis acuta (SchK.) PRFsL, Hook. Sp. Fil. 4 (1862) 153;

VOLKENS 1. c. 453.

Yap (VOLKENS). Distrib. In both hemispheres.

Nephrolepis acutifolia (Desv.) Chrisl, in Verh. Nat. Ges, Basel.

11 (1905) 243; MERR. 1. c. 44.

Lindsaya acutifolia DESV. Prodr. (1827) 312.

Palau 101; Ponape Kolonia 724. In forests at low altitudes. Distrib. Tropical Africa through Malaya, Burma to Australia.

Nephrolepis biserrata SchorT Gen. Fil. (1834) t. 3.

Asplenium biserratum Sw. Schrad. Journ. (1801) 32. 
Palau 264, 3308; Ponape 687 ; Kusai 1450, 1451; Rota 1799. Distrib. In both hemispheres. Jap. name: Hôbi-kwanzyu.

Nephrolepis hirsutula (ForST. f.) PRESL Tent. (1836) 79; MrRr. 1. c. 44 ; KoIDz. in Bot. Mag. Tokyo 29 (1915) 245.

Polypodium hirsutulum Forst. f. Prodr. (1786) 81.

Guam (MerriLl); Jaluit (KoIdzumi). Distrib. Tropics of both hemispheres.

\section{Odontosoria FÉE}

Odontosoria chinensis (LinN.) J. SM, in Bot. Voy. Herald (1857) 430 ; MERR. I. c. 44.

Trichomanes chinensis. LinN. Sp. Pl. (1753) 1099.

Guam (Merrill). Distrib. Tropical Asia to Madagascar and Poly. nesia. Jap. name: Hamahôrai-sinobu.

Odontosoria retusa (CAv.) J. SM. in Bot. Voy. Herald (1857) 430; MERR. 1. c. 45.

Davalia retusa CAV. Descr. (1902) 273.

Guam. (MerriLl). Distrib. Malaya and Polynesia.

\section{Polypodium Linnael's}

Polypodium accedens Bi. Enum. (1828) 121.

Ponape 805 ; Kusai 1385. Distrib. Malaysia to Polynesia.

Polypodium decorum Brack. Expl. Ex. 16 (1854) 7, t. 2. f. 2.

Palau 496, 2352. Distrib. Ceylon, Malaysia and Polynesia.

Polypodium nigriscens Blume Enum. (1828) 126.

Ponape 617, 628. Distrib. India, Ceylon, Malaysia, Polynesia and tropical Australia.

Polypodium obliquatum BL. Ènum. (1828) 128.

Palau 494. Distrib. India, Ceylon, Malaya and Philippines. Jap. name: Naname-sida.

Polypodium persicifolium DEsv. Berl. Mag. 5 (1811) 316.

Ponape 809, 1612. In forests at high altitudes. Distrib. Java and Celebes.

Polypodium phymatodes LINN. Mant. (1771) 306; Volkens 1. c. 553 ; MeKR. 1. c. 45 ; KoIDz. 1. c. 245. 
Palau 84; Truk 620; Guam (MerRILL); Yap (VolKens); Jaluit (KoIDzumi). Distrib. Tropical Africa and $\Lambda$ sia to Australia and Polynesia.

Polypodium punctatum (LINN.) Sw. in Schrad. Journ. 1800,2 (1801) 21 ; MERR. 1. c. 45.

Acrostichum punctatum LiNN. Sp. Pl. ed. 2 (1763) 1524.

Saipan 991; Guam (Merrill). Distrib. Tropiscal Africa, Asia through Malaya to Polynesia.

\section{Polysctichum RoTH}

Polystichum aculeatum (Forst.) PresL Tent. (1836) 83.

Polypoditum aculeatum Forst. Prodr. (1786) 82.

Ponape 1617. In forests at high altitudes. Distrib. India to Japan through Malaya lo Polynesia and also in South Africa.

\section{Prosaptia PResL}

Prosaptia alata (BL.) Christ. Ann. Bot. Buit 2, 5 (1905) 127.

Davalia alata BL. Enum. (1828) 230.

Kusai 1377. Distrib. Tropical Asia to Samoa.

\section{Pteris Linnaeus}

Pteris biaurita LinN. Sp. Pl. (1753) 1076; Merr. 1. c. 45.

Guam (Merrill). Distrib. Warmer parts of both hemispheres. Jap. name: Kokesida.

Pteris ensiformis Burm. Hook. Sp. Fil. 2 163; VolKens 1. c. 453. Palau 485, Yap 1124. In forests at low altitudes. Distrib. Hindstan, Himalaya, Ceylon, Australia, Polynesia and Formosa. Jap. name: Hoko-sida.

Pteris pellucida PREsl Rel. Haenk. 1 (1825) 55.

Ponape 838, 1643. Distrib. India, Java and Philippines.

Pteris quadriaurita Retz. Obs. 6 (1791) 38; Volkens 1. c. 453; MERR. 1. c. 45.

Ponape 821; Kusai 1454; Anatahan 2145, 4149; Yap (VoLKeNS); Guam (MerriLl). Distrib. Warmer parts of both hemispheres. Jap. name: Hatizyô-sida. 
Pteris tripartita Sw. in Schrad. Journ. 1800, 2 (1801) 67; MerR. I. c. 45 .

Palau 590; Saipan 37; Guam (MrRILl). Distrib. Tropical Africa and Asia to Polynesia. Jap. name: Heridori-warabi.

\section{Schizoloma Gaudichaud}

Schizoloma ensifolium (Sw.) J. Sm. in Hook. Journ. Bot. 3 (1841) 414 ; Merr. 1. c. 45.

Lindsaya ensifolia Sw. in Schrad. Journ. 1800, 2 (1801) 77.

Palau 413. Distrib. Tropical Africa and Asia to Polynesia. Jap. name: Inu-inomotosô.

\section{Taenitis WILLDENOW}

Taenitis blechnoides (WILLD.) Sw. Syn. 24 (1806) 220.

Palau 280, 307, 2309. Distrib. Tropical Asia through Malaya to the Philippines and Fiji.

\section{Tapenidium (PresL) C. Christensen}

Tapenidium pinnatum (CAv.) C. CHR. Ind. (1905) 213.

Davalia pinnata CAv. Desc. (1802) 277.

Ponape 795, 1549, 1615; Palau 2325. Distrib. Malaysia and Polynesia.

\section{Tectaria Cavanilles}

Tectaria crenata CAV. Desc. (1802) 250; MerR. 1. c. 45.

Saipan 27, 998, 2221; Yap 1129; Rota 1797. Distrib. Malaya.

Tectaria grandifolia (PReSL) CopeL. in Philip. Journ. Sci. 2 (1907) Bot. 413.

Asplenium grandifolium PRESL Epim. (1851) 64 .

Ponape 844. Distrib. Philippines and the Santa Crucz Islands.

Tectaria irregularis (PRESL) COPEL. 1. c. 416.

Polypodium irregulare PRESL Rel. Haenk 1 (1825) 25 t. 4, f. 3.

Aspidium irregulare C. CHR. Ind. (1905) 78.

Palau 267, 532, 2083. In forests at low altitudes. Distrib. Malaya and Philippines.

Tectaria polymorpha (WALL.) COPEI. I. c. 413.

Asplenium polymorphum WaLI. List No. 382 (1828), Hook. Sp. Fil.

4 (1862) 54 . 
Palau 462; Ponape 822. Distrib. India to Malaysia and the Philip. pines.

\section{Vittaria SMITH}

Vittaria elongata Sw. Syn. (1806) 109, 302 ; Volkess 1. c. 453 ; MERR. 1. c. 45.

Palau 325, :1950, 2334; Saipan 988; Yap 1259. Distrib. Tropical Asia to Polynesia.

\section{Parkeriaceae}

\section{Ceratopteris BRONGNIART}

Ceratopteris thalictroides (LINN.) BRONGN. Bull. Soc. Philom. (1821) 186 ; VOLKENS 1. c. 454 ; MerR. 1. c. 46.

Acrostichum thalictroides LrnN. Sp. P1. ed. 2 (1763) 1488.

Palau 484 ; Yap 1245. In swamps. Distrib. Tropics of both hemispheres. Jap. name: Mizu-warabi.

\section{Gleicheniaceae \\ Gleichenia SMrTH}

Gleichenia linearis (Burm. f.) Clarke in Trans. Nat. Soc. Bot. 1 (1880) 428 ; Volkens 1. c. 454 ; Merr. 1. c. 46.

Polypodium lineare Burm. f. Fl. Ind. (1768) 235, t. 67, f. 2.

Palau 345; Yap 1150; Kusai 1447. Distrib. Warmer parts of both hemispheres. Jap. name: Kosida.

\section{Schizaeaceae}

\section{Lygodium SWARTZ}

Lygodium circinatum (Burm. f.) Sw. Syn. (1806) 153; Volkens 1. c. 454 .

Yap (VolKENS); Palau 134. Distrib. India to southern China through Malaya to Australia.

Lygodium fiexuosum (LiNN.) Sw. Schrad. Journ. (1801) pt. 106; VoLKENS 1. c. 454.

Ophioglossum flexuosum LinN. Sp. Pl. (1753) 1063.

Yap (Volkens). Distrib. Southern China, Malaysia, Philippines to Queensland. Jap. name: Sima-kamikusa. 
Lygodium scandens (LINN.) Sw. in Schrad. Journ. (1801) 106; VOLKENS 1. c. 454 ; MERR. 1. c. 46.

Palau 85; Yap (Volkens); Guam (Merrill). Distrib. Old World Tropics generally. Jap. name: Iriomoté-syamisenzuru.

Lygodium semihastatum (CAv.) DEsv. Prodr. (1827) 203; MERR. I. c. 46 .

Ugena semihastata CAV. Ic. 6 (1801) 74, t. 594, f. 1.

Guam (Merrill). Distrib. Philippines.

\section{Schizaea SMITH}

Schizaea dichotoma (LinN.) SM. Mém. Acad. Turin 5 (1793) 422, t. 9, f. 9 ; Volkens l. c. 454 ; Merr. in Philip. Journ. Sci. 15 (1919) Bot. 539.

Acrostichum dichotomum LinN. Sp. Pl. (1753) 1068.

Palau 279, 2037, 2087, 2330; Yap 1143; Guam (MERrILL). Distrib.

Asia, tropical Australia, Madagascar to Mascarene Islands.

Schizaea digitata (LINN.) Sw. Syn. (1806) 160, 380 t. 4, f. 1; VolKENS 1. c. 454.

Achrosticum digitatum LinN. Sp. Pl. (1753) 1068.

Palau 465. Distrib. Tropical Asia to Madagascar.

\section{Lycopodiaceae}

\section{Lycopodium LINNAEUS}

Lycopodium cernum LinN. Sp. Pl. (1753) 1103 ; Volkens 1. c. 454; MERR. 1. c. 47.

Palau 154; Ponape 645, 1616; Yap 1151; Kusai 1468; Aramagan 2193. Distrib. Tropics of both hemispheres. Jap. name: Mizusugi.

Lycopodium phlegmaria LINN. Sp. Pl. (1753) 1100; MERR. 1. c. 47. Palau 268, 464, 537 543, 2358; Ponape 818, 1647 ; Kusai 1316, 1374, 1438. Distrib. Tropical Asia to Polynesia.

Lycopodium squarrosum ForsT. Prodr. Fl. Ins. Austr. (1797) No 479.

Palau 493. Distrib. Pantropic.

\section{Selaginellaceae}

Selaginella SPRING 
Selaginella belangeri (BOKY) SPRING Mono. Lycopod. 2 (1850) 242; MERr. 1. c. 47.

Lycopodium belangeri Bory in BÉl.ANG. Voy. Bot. 2 (1846) No. 16, t. 2, f. 3.

Guam (MERRILL). Distrib. Indo-malayan region.

Selaginella Kanehirae Alston in Journ. Bot. 72 (1934) 227.

Ponape 732, 760, 1649. Distrib. Endemic.

Selanginella Volkensii HIERON. in ENGL. Bot. Jahrb. 52 (1914) 1.

Selaginella Menziesii (non SPRING) VolKens 1. c. 455.

Yap 1144. Distrib. The type from Yap, endemic.

\section{Psilotaceae}

\section{Psilotum SWARTZ}

Psilotum complanatum Sw. Syn. Fil. (1806) 188, 414, t. 4, f. 5.

Palau, 536; Kusai 1444. Distrib. Jamaica, Mexico, Tahiti, Java and Singapore.

Psilotum nudum (Linn.) Griseb. Syst. Veg. Karaib. (1857) 130; MERR. 1. c. 47.

Lycopodium nudum LINN. Sp. Pl. (1753) 1100.

Palau 103, 551, 1921; Rota 1814. Distrib. Tropics of both hemispheres.

Psilotum triquetrum Sw. Syn. Fil. (1806) 187 ; Baker Handb. Fern Allies (1887) 30 ; VolKens 1. c. 454.

Yap (Volkens). Distrib. Pantropic. Jap. name: Matubaran.

\section{SPERMATOPHYTES}

Cycadaceae

\section{Cycas Linnaeus}

Cycas circinalis Linn. subsp. Seemannii (A. Braun) Schuster in Pflanzenr. Heft 99 (IV, 1) (1932) 71 ; KanehIRA Fl. Micr. (1933) 59 .

Cycas Seemannii A Braun in Sitzungsber. Ges. Naturf. Freunde Berlin (1876) 114. 
Cycas circinalis (non Linn.) Merr. in Philip. Journ. Sci, 9 (1914) Bot. 48.

Cycas Rumphii (non MiQ.) KanehIRA in Bot. Mag. Tokyo 45 (1931) 273.

Yap 1189; Palau 233, 2048; In primary forests. Distrib. Fiji to Amboina. Nat. names: "fratel" or "frotel" (Yap), "rumiyan" (Palau).

\section{Pandanaceae \\ Freycinetia Gaudichaud}

Freycinetia almonoguiensis KANEHIRA in Bot. Mag. Tokyo 49 (1935) 186 f. 18.

Palau, Almonogui, 2775! S. NisidA. Distrib. Endemic.

Freycinetia carolinensis KANEHIRA op. cit. 185. f. 17.

Palau, Aimiriik 2359! S. Nisida. Distrib. Endemic.

Freycinetia mariannensis MerR. in Philip. Journ. Sci. 9 (1914) Bot. 48. Kanehira in Bot. Mag. Tokyo 49 (1935) 185.

The type from Guam. Saipan 2243, Aramagan 2196. Distrib. Endemic.

Freycinetia ponapensis MarTelli in Bot. Mag. Tokyo 48 (1934) 129.

Ponape, Patapat, Ledermann 13245. Distrib. Endemic.

Freycinetia Villalobosensis MARTELLI in Bot. Mag. Tokyo op. cit. 128; KanehiRa 1. c. 185.

Palau, Babeldaob, Ledermann 14323 ; Palau 3340, 3441 (Kanehira) Distrib. Endemic.

\section{Pandanus Linnaeus}

Pandanus aimiriikensis MARTeI.I in KanfhiRA FI. Micr. (1933) 60, f. 1, nom. seminud., Bot. Mag. Tokyo 48 (1934) 125, f. 7.

Palau Aimirrik 328! In thickets at low altitudes. Distrib. Endemic.

Pandanus brachypodus KaneHIRA in Bot. Mag. Tokyo 49 (1935) 425 , f. 32 .

Enchabi Kusai 3480 ! Distrib. Endemic. Nat. name: "punmusi" (Enchabi). 
Pandanus carolinianus MarTelli in Webbia 4, 2 (1914) 400, t. 34, f. 1-3; KANEHIRA in Bot. Mag. Tokyo 49 (1935) 61, f. 2.

The type from Yap. Yap 3343. Distrit. Endemic.

Pandanus Cominsii Hemsl. Hook. Icon. (1900) 2654; Martelu. in ENGL, Bot. Jahrb, 49 (1912) 66, Webbia 4 (1914) t 26, f. 20; KANEHIRA op. cit. 49 (1935) 356, f. 31.

The type from the Solomon Islands. Truk 3489. Distrib. The Solomon, Bismarck and New Hebrides.

Pandanus cylindricus Kakehira in Bot. Mag. 'Tokyo 49 (1935) 63, f. 3 .

Ponape 2810, Mokiel 3461. Distrib. Endemic Nat. name: "silaue" (Ponape).

Pandanus cylindricus var. Sinnau KaneHIRA in Bot. Mag. Tokyo 49 (1935) 356, f. 30.

Truk, Tol 3448! Distrib. Endemic. Nat. name: "sinnau" (Truk).

Pandanus dilatatus KaneHIRA in Bot. Mag. Tokyo op. cit. 63, f. 4. Ponape, Nutt 2808! Distrib. Endemic. Nat. name: "kienpel" (Ponape).

Pandanus divergens KanehirA in Bot. Mag. Tokyo 49 (1935) 60, f. 1.

Palau, Airai 2389! Distrib. Endemic.

Pandanus dubius Spreng. Syst. 3 (1826) 897 ; Kurz in Journ. Bot. 5 (1867) 127 , t. 64 . f. 1 \& 2 ; WARB. in KRIEGLR, Neuguinea (1899) t. 7, ENGL. Pflanzeur. (Pandanaceae) (1900) 50, f. 1, f. 14 A-D; Martelli, Webbia 4 (1913) 12; Merr. Philip. Journ. Sci. 9 (1914) Bot. 48; Kanehira in Bot. Mag. Tokyo 45 (193i) 273, Fl. Micr. (1933) 61, f. 2.

Ponape 877, Saipan 900, Kusai 3507, Truk 3494, Saipan 3530. Distrib. India to New Hebrides. Nat. names: "pafung "(Saipan), "poak" (Truk), " poko" (Palau) "meu yok" (Kusai).

Pandanus duriocarpoides KaneHIRA in Bot. Meg. Tokyo 49 (19335) (ined.)

Yap, Tomil 3497! Distrih. Endemic. Nat. Name:

Pandanus duriocarpus Martelli in Kanehira Fl. Micr. (1933) 62, f. 3, nom. seminud., Bot. Mag. Tokyo 48 (1934) 116, f. 1.

Palau Babeldaob 882 ! Distrib. Endemic. 
Pandanus enchabiensis KANEHIRA in Bot. Mag. Tokyo 49 (1935) 429 , f. 36 .

Enchabi 3470! Distrib. Endemic. Nat. name: "moak" (Enchabi).

Pandanus erythrophloeus KANEHIRA in Bot. Mag. Tokyo 49 (1935) 66 , f. $5 \& 6$.

Palau, Peliliu 2419! In thickets near the seashore. Distrib. Endemic.

Pandanus Eyesyes Kanehira in Bot. Mag. Tokyo 49 (1935) (ined.). Kusai 3503! Distrib. Endemic. Nat. name: "eyesyes" (Kusai).

Pandanus fragrans Gaudich. Voy. Bonite (185̃1) t. 22, f. 10; Merr. 1. c. 48 ; Martelli in Bot. Mag. Tokyo 48 (1934) 117, f. 2. The type from Guam; Saipan 2132 b. Distrib. Endemic.

Pandanus guamensis Martelli in Webbia 4, 1 (1914) 404, t. 42, f. 4-6; Merr. 1. c. 48 ; Kanehira in Bot. Mag. Tokyo 49 (1935) 67, f. 7.

Saipan 3340. The type from Guam. Distrib. Endemic.

Pandanus Hollrungii WARB. forma caroliniana MARTELI.I in ENGI. Bot. Jahrb. 49 (1912) 66.

The type from Tol, Uman, Truk (Krasmfr). Distrib. Endemic.

Pandanus Hosinoi KaneHIrA in Bot. Mag. Tokyo 49 (1935) 103, f. 8 .

Ponape 2811! 3450; Makiel 3458. Distrih. Endemdic. Nat. name: " nane kejak" (Ponape) "nenketak" (Mokiel).

Pandanus insularis KANEHIRA in Bot. Mag. Tokyo 49 (1935) (ined.). Kusai 3504. Distrib. Endemic. Nat. name: "meu kosa" (Kuasai).

Pandanus jaluitensis KANEHIRA in Bot. Mag. Tokyo 49 (1935) 103, f. 9 , ibid. 190.

Jaluit Emedi 2788 ! 2791; Kusai 2803-5 ; Ponape 3453, 3456 ; Mokiel 3463. Nat. names: "joene" "tibitin" (Jaluit), "pacheren" "musha-musi" (Ponape), "feufol" (Kusai) "bederik" (Mokiel).

Pandanus japensis Martelli in Engl. Bot. Jahrb. 49 (1912) 65.

The type from Yap (Volkens). Distrib. Endemic.

Pandanus kafu Martelli in Webbia 4 (1914) 405, t. 19. f. 1-3; Merr. 1. c. 47; Kanehira in Bot. Mag. Tokyo 45 (1931) 273, F1. Micr. (1933) 62, f. 4.

Saipan 936. The type from Guam. Distrib. Endemic. Nat. name: "kafu" (Guam, Saipan). 
Pandanus Kanehirae Martelli in KaneHiRa Fl. Micr. (1933) 65, f. 5, nom. seminud., Bot. Mag. Tokyo 48 (1934) 119, f. 3.

Palau, Babeldaob 445! 1987, 3342. Distrib. Endemic. Nat. name: "bôk" (Palau).

Pandanus korrensis Kanehira in Bot. Mag. Tokyo 49 (1935) 106, f. 10 .

Palau Korror 3348! S. NisIDA. Distrib. Endemic. Nat. name: "syu" (Palau).

Pandanus kusaiensis Kanehira in Bot. Mag. Tokyo 49 (1935) 107, f. 11.

Kusai 2796! 2806. Distrib. Endemic. Nat. name: "kosbia" (Kusai).

Pandanus Lakatwa KaneHIRA in Bot. Mag. Tokyo 49 (1935) (ined.) Jaluit 3516! Nat. name: "lakatwa" (Jaluit).

Pandanus laticanaliculatus KANEHIRA in Bot. Mag. Tokyo 49 (1935) 188, f. 19.

Jaluit 2801 ! Distrib.. Endemic. Nat. name: " jonmouia" (Jaluit).

Pandanus laticanaliculatus var. edulis KANEHIRA ibid. 188.

Jaluit 2786; Distrib. Endemic. Nat. name: "erwan" (Jaluit).

Pandanus macrocephalus KANEHIRA in Bot. Mag. Tokyo 49 (1935) 428.

Mokiel 3457; Jaluit 2794, 2797. Distrib. Endemic. Nat. names: “intekul" (Mokiel), "Cernenu" (Joluit).

Pandanus macrojeanneretia Martelle in KanehiRa Fl. Micr. (1933) 66, f. 6, nom. seminud. Bot. Mag. Tokyo 48 (1934) 121, f. 5.

Palau Aimiriik 1976! In thickets at low altitudes. Distrib. Endemic.

Pandanus Menne KanehiRa (ined.)

Jaluit 3517! 3522. Nat. name: "menne" (Jaluit).

Pandanus obliquus KanehiRA in Bot. Mag. Tokyo 49 (1935) (ined.). Jaluit 3525 ! 3512, 3518, 3529. Nat. names: "lajokorer," "lonlin," jominjekar" "lamtir" (Jaluit).

Pandanus odontoides Hosokawa in Trans. Nat. Hist. Soc. Formos. 24 (1934) 197, f. 2 \& 4.

The type from Alkoln, Palau. Distrib. Endemic.

Pandanus odoratissimus var. acutus KANEHIRA nom. nov.

Pandanus tectorins var. acutus KANEHIRA in Bot. Mag. Tokyo 49 (1935) 194. 
Pandanus sinensis (non Martelli) KanehiRa Fl.Micr. (1933) 69, f. 8. Palau, Korror 1896, 2137. Distrib. Endemic. Nat. name: "ongor" (Palau).

Pandanus odoratissimus var. angaurensis KANEHIRA nom. nov.

Pandaus tectorius var. angaurensis KANEHIRA in Bot. Mag. Tokyo 49 (1935) 193, f. 22.

Palau Angaur 3346. Distrib. Endemic.

Pandanus odoratissimus var. Fatyanion KANEHIRA nom. nov.

Pandanus tectorinus var. Fatyanion KANEHIRA ibid. 194, f. 23.

Truk 2817! Distrib. Endemic. Nat. name: "fatyanion" (Truk).

Pandanus odoratissimus var. Ongor KANEHIRA nom. nov.

Pandanus tectorius var. Ongor KaneHIRA ibid. 194, f. 24.

Palau, Korror 3347! S. NisIDA. Distrib. Endemic. Nat. name: "ongor" (Palau).

Pandanus Okamotoi Kanehira ibid- 107, f. 12.

Palau Aimirrik 2374! In waste grass land. Distrib. Endemic.

Pandanus palawensis MarTelll in Bot. Mag. Tokyo 48 (1934) 124 , f. 6.

Type from Palau, Babeldaob Ledermann 14373. Distrib. Endemic.

Pandanus palkilensis Hosokawa in Trans. Nat. Hist. Soc. Formos. 24 (1934) 197.

Panape Palkier. The syncarps of the type is immature, it is probably a synonym of Pandanus ponapensis MarTellr.

Pandanus patina Martelli in Kanehira Fl. Micr. (1933) 67, f. 7 , nom. seminud., Bot. Mag. Tokyo 48 (1934) 127, f. 8.

Ponape Nanaraut-zan 1668 ! In thickets at about $700 \mathrm{~m}$ altitude. Distrib. Endemic. Nat. name: " peet" (Ponape).

Pandanus peliliuensis KaneHrRA in Bot. Mag. Tokyo 49 (1935) 113 , f. 15 \& 16.

Palau, Peliliu 2421! In thickets at low altitudes. Distrib. Endemic.

Pandanus ponapensis MarTelli in Bot. Mag. Tokyo 48 (1934) 121, f. 4.

Ponape 2134 ! 3448. Distrib. Endemic. Nat. names: "kipal " "alwan" (Ponape).

Pandanus pulposus Martelli in Webbia 4, 2 (1914) 409, t. 38, f. 1.3; Forest Brown Occ. Papers Bishop Mus. Honolulu 9, 
No. 4 (1930) 3; KanehrRA in Bot. Mag. Tokyo 49 (1936) 110, f. 13 .

Pandanus tectorius var. pulposus Warb. in ENGL. Pflanzenr. Heft. 3, IV, 9 (1900) 49.

Jaluit, Emedi 2787. The type from Radak, Marshall Islands. Dis. trib. Endemic. Nat. name: "jilebar" (Jaluit).

Pandanus rectangulatus KANEHIRA (ined.)

Enchabi 3469, 3477, 3479, 3483. Nat. names: "olwo," "demesyo," " anilip," " pathaplip" (Enchabi).

Pandanus rhombocarpus KANEHIRA 1. c. 49 (1935) 426 f. 33.

Enchabi 3467! 3463. Distrib. Endemic. Nat. name: "poppaniwa" (Enchabi).

Pandanus rotundatus KanehiRA 1. c. 49 (1935) 427. f. 35.

Ponape 3449 ! 3515. Distrib. Endemic. Nat. name: "majojokjok" (Ponape).

Paudanus trukensis Kanehira in Bot. Mag. Tokyo 49 (1935) 190, f. 21.

Truk 2816! Kusai 2792, Jaluit 2789. Distrib. Truk, Kusai to Jaluit; endemic. Nat. names: "meu-oa" (Kusai), "mojel" (Jaluit).

Pandanus Utiyamai KaneHIRA in Bot. Mag. Tokyo 49 (935) 190, f. 20 .

Ponape 2809! Distrib. Endemic. Nat. name: "jajeperik" (Ponape).

Pandanus Volkensii KanehIRA in Bot. Mag. Tokyo 49 (1935) 111, f. 14.

Yap 2748! 3345. Distrib. Endemic.

\section{Potamogetonaceae}

Diplanthera THOUARS

Diplanthera uninervis (FORSK.) Aschers in ENGL. \& PRANTL Nat. Pflanzenf. Nachtr. 1 (1897) 37; MrRR. 1. c. 49.

Zostera uninervis Forsk. Fl. Aeg.-Arab (1775) 159.

Guam. Disirib. In shallow salt water, from the Red Sea and tropical East Africa and to Polynesia. 
Potamogeton Iucens LiNN. Sp. Pl. (1753) 126 var. Merr. 1. c. 49. Potamogeton Gaudichaudii CHAM. et SchilechT. in Linnaea 2 (1827) 199.

Guam. Distrib. The variety endemic.

Potamogeton mariannensis CHAM. et SCHLECHT. 1. c. 2 (1827) 228. MERR. 1. c. 49.

Guam. Distrib. Endemic.

\section{Ruppia LinnaEus}

Ruppia maritima Livn. Sp. Pl. (1753) 127; MERr. 1. c. 50.

Guam. Distrib. Widely distributed in warm countries, growing in brackish water.

\section{Hydrocharitaceae}

Enhalus L. C. RICHARD

Enhalus acoroides (LinN. f.) Rich, ex Chatin Anat. Pl. Aquat. (1862) 15, t. 6 ; Volkens 1. c. 455 ; Merr. 1. c. 50.

Stratiotes acroides LINN. f. Suppl. (1781) 268.

Guam, Yap. Distrib. Widely distributed in the Indo-Malayan region in salt water.

\section{Blyxia Thouars}

Blyxia octandra (Roxb.) Planch. ex Thwaites Enum. Pl. Ceyl. (1859-64) 332 ; VOLKENS 1. c. 455.

Vallisneria octandra RoxB. PI. Coromand. 2 (1798) 34, t. 165.

In rice paddies at low altitudes. Yap 1244, Palau 483. Distrib. Tropical Asia to Malaya, Australia and the Carolines. Jap. name: Nanyôsubuta.

\section{Halophila Thounds}

Halophila ovata Gaudich. Bot. Freyc. Voy. (1826) 430, t. 40, f. 1 ; MERR. I. c. 50.

Guam. Distrib. Guam and Luzon.

\section{Thalassia BANKS}

Thalassia Hemprichii (EHrb.) Aschers in ENGL. \& PRANTt. Nat. Pflanzenf. 2, 1 (1889) 254; Volkens 1. c. 455.

Schizotheca Hemprichii EHREnB. in App. Akad. Berl. 1 (1832) 429.

Yap (Volkens), Distrib. India to Polynesia. 


\section{Gramineae}

\section{Andropogon LinNAEUS}

Andropogon aciculatus ReTz. Osb. 5 (1789) 22 ; VolKENS 1. c. 456; MERR. 1. c. 53.

Yap. Distrib. India to China southward through Malaya to tropical Australia.

Andropogon brevifolius Sw. Prodr. (1788) 26; VoLKENS 1. c. 456. Guam, Yap. Distrib. Pantropic.

Andropogon eitratus DC. Cat. Hort. Monsp. (1813) 78; MERR. 1. C. 53.

The citronella grass. A native of tropical Asia, now widely cultivated for its fragrant leaves.

Andropogon contortus LinN. Sp. Pl. (1753) 1045; MERR. 1. c. 53. Guam. Distrib. Pantropic.

Andropogon halepensis (LinN.) BRot. Fl. Lusit. 1 (1804) 89, var. propinquus (KunTH) Merr. in Philip. Journ. Sci. 1 (1906) Suppl. 336, 9 (1914) Bot. 53.

Holcus halepensis LiNN. Sp. Pl. (1753) 1047. Andrpogon propinquus KunTH Enum. 1 (1833) 502.

Saipan 911, 965; Palau 212; Guam (MerRILL). Distrib. The species pantropic, the variety from Ceylon to Moluccas.

Andropogon intermedius R. BR. Prodr. (1810) 202; VolKFns 1. c. 456.

Yap. Distrib. China, Philippines to Australia.

Andropogon Sorghum (LINN.) BRoT. Fl. Luist. 1 (1804) 88; Merr. l. c. 53 .

Holcus Sorghum Lin's. Sp. Pl. (1753) 1047.

Guam (MERRILL). Cultivated in all warm countries.

\section{Bambusa SchreBer}

Bambusa spinosa Roxis. Hort. Beng. (1814) 25.

Bambusa blumeana Schultes f. Syst, 7, 2 (1830) 1343 ; Merr. I. c. 58 ; Kanehira in Bot. Mag. Tokyo 45 (1931) 273.

Palau, Korror 171; Guam (Merkill). A spiny bamboo, planted in 
villages. Distrib. Southern China to the Malay Peninsula and Archipelago to the Moluccas.

Bambusa vulgaris Schrad, ex. WeNDL. Collect. Pl. 2 (1808) 26, t. 47 ; Merr. 1. c. 58 ; KanehiRA 1. c. 273.

Truk 624; Ponage 861; Saipan 903; Yap 1179; Kusai 1365, 1421. Distrib. Patropic in cultivation, Nat. name: "alkasrem" (Kusai).

\section{Cenchrus Linnaeus}

Cenchrus calyculatus CAv. Icon. 5 39, t. 463; KoIDz. in. Bot. Mag. Tokyo 29 (1915) 247.

Jaluit. Distrib. Polynesia.

Cenchrus echinatus Livn. Sp. Pl. (1753) 1050 ; Merr. 1. c. 56.

Guam. Distrib. A native of tropical America, now naturalized in other tropical regions.

Cenchrus viridis Spreng. Syst. Veg. 1 (1825) 301.

Cenchrus echinatus PREsL Rel. Haenk. 1 (1830) 317.

Palau, Angaur 570; Saipan 961, 1042. Distrib. A native of tropical America, now also found in Guam, Siam, Indo-China, Philippines, New Guinea and the northern Australia.

Centhotheca Desvaux

Centhotheca latifolia (OsBeck) TRIN. Fund. Agrost. (1820) 141; MERR. 1. c. 58.

Holcus latifolius OsBeck Dagbok Ostind. Resa (1757) 427.

Centhotheca lappacea Desv. in Nouv. Bull. Soc. Philom. 2 (1810) 189 ; VOLKENS 1. c. 457.

Palau: Korror 128, Aimirik 300 ; Ponape 767, 881; Yap (VoLKENs); Kusai 1692. Very common in thickets, forests and clearings at low altitudes. Distrib. Tropical Asia and Africa through Malaya to Australia and Polynesia.

\section{Coix Linnaeus}

Coix lachryma-jobi LinN. Sp. Pl. (1753) 972; MrRR. l. c. 50.

Guam. Distrib. Tropics of both hemispheres, probably a native of India. Jap. name: Zyudu-dama.

\section{Cynodon Persoon}


Cynodon Dactylon ((Linn.) Pers. Syn. 1 (1804) 85; Volkens 1. c.

457; Merr. 1. c. 57.

Panicum Dactylon LinN. Sp. (1753) 58.

Yap (Volkens), Guam (Merrill). Distrib. Pantropic.

\section{Dactyloctenium WILLDENOW}

Dactyloctenium aegyptiacum (Linn.) Willd. Enum. Hort. Berol. (1809) 1029 ; Volkens 1. c. 457 ; Merr. 1. c. 57.

Cynosurus aegyptius Livn. Sp. Pl. (1753) 72.

Saipan 982, Yap (Volkens), Guam (Merrill.). Distrib. Warmer parts of both hemispheres.

\section{Dimeria R. BRown}

Dimeria chloridiformis (GaudiciI.) K. Schum. \& LaUterb. Fl. Deutsch. Schutzg. Südsee (1901) 165; MLRR. 1. c. 50.

Andropogen chloridiformis GaudicH. Bot. Freyc. Voy. (1826) 412.

Guam. Distrib. Endemic.

Dimeria fuscens Trin. in Mém. Acad. Sci. St. Pétersb. Sér. 6, 2 (1833)335; VOLKENS 1. c. 455.

Yap (Volkens). Distrib. East Indies.

Dimeria ornithopoda .TRIN. Fund. Agrost. (1820) 167, t. 14 ; MERr.

l. c. 51, var. tenera (TRIN.) HACK. in DC. Monog. Phan. 6 (1889)

81; Merr. in Philip. Journ. Sci. 1 (1906) Suppl. 321.

Dimeria terena TRIN. in Mém. Acad. Sci. St. Pétersb. 6, 2 (1833) 335 ; VOLKENS 1. c. 455.

Guam, Yap. Distrib. India to Japan southward to Australia.

\section{Eleusine GAERTNER}

Eleusine indica (Linn.) Gaerta. Fruct. 1 (1788) 8; Volikens 1. c. 457 ; Merr. 1. c. 57.

Cynosurus indicus LinN. Sp. Pl. (1753) 72.

Palau, Korror 113; Yap; Guam. Distrib. All warm countries.

Eriachne R. BROwN

Eriachne pallescens R. BR. Prodr. (1810) 184; Volkens 1. c. 456. Yap. Distrib. India to southern China through Malaya to Australia. 


\section{Eragrostis BeuvoIS}

Eragrostis amabilis (LINN.) W. ct A. in HoOK. et ARN. Bot. Beechy Voy. (1841) 251 ; Mrerr. Enum. Philip. Fl. Pl. 1 (1922) 88.

Poa amabilis LinN. Sp. Pl. (1753) 68.

Eragrostis tenella Roem. et Schult. Syst. 2 (1817) 576; Volkens l. c. 457 ; Merr. 1. c. 57.

Metaranium, Ponape 776; Yap (Volkenss) ; Guam. Distrib. Warmer parts of both hemispheres.

Eragrostis pilosa (LiNN.) Beauv. Agrost. (1812) 71; Merr. 1. c. 57. Poa pilosa Linn. Sp. PI. (1753) 68.

Guam (Merrill). Distrib. Pantropic.

Eragrostis plumosa Link, Hort. Boerl. 1, 192; Volkens 1. c. 457. Yap. Distrib. Tropical Asia and Africa.

\section{Garnotia BRongniART}

Garnotia stricta BRONGN. in Bot. DuperRy's Voy. (1829) 132, t. 21 ; MrRr. in Philip. Journ. Sci. 15 (1919) Bot. 540.

Guam (MiskillL). Distrib. India through Malaya to Hawaii.

\section{Imperata CYRILLO}

Imperata exaltata Brogx. Voy. Coq. Voy. Bot. (1929) 101, exc. syn. RoxB., Volkens 1. c. 455.

Yap (Vorkens). Distrib. Philippines to the Moluccas.

Isachne R. BROWN

Isachne globosa (Thuns.) OK. Rev. Gen. Pl. (1891) 778; MFrr. Enum. Philip. Fl. Pl. 1 (1922) 59; Hosokawa in Trans. Nat. Hist. Soc. Formos. 24 (1934) 200.

Milium globosum Thunb. Fl. Jap. (1784) 49.

Palau (Hosokawa). Distrih. Australia, India, Malaya to China and the Philippines.

Iscachne miliacea RotIi, Nov. Sp. (1821) 58; VolKens 1. c. 456; MERR. 1. c. 55

Isachne minutula KunTH, Rev. Gram. 2 (1829) t. 117, Enum. 1 (1833) 137; VOLKENS 1. c. 456.

Guam, Yap. Distrib. India to Malaya and Polynesia. 
Isachne ponapensis Hosokawn in 'Trans. Nat. Hist. Soc. Formos. 24 (1934) 200.

Ponape (Hosokawa). Distrib. Endemic.

Isachne rigida NfFs ex Steud. Syn. Pl. Gram. (1826) 95; VoLKENS. 1. c. 456.

Yap (VolKens). Distrib. Java.

\section{Ischaemum LinNaEus}

Ischaemum chordatum (Trin.) HACK. ex. WARB. in ENGL. Bot. Jahrb. 13 (1891) 260; MrrR. 1. c. 52.

Spodipogon chordatus Trin. in Mèm. Acad. Pétersb. 6, 2 (1833) 302.

Guam. Distrib. New Guinea.

Ischaemum digitatum BRONGN. Bot. DuperreY's Voy. (1929) 70, t. 13 ; VolKens 1. c. 466 , var. polystachyum (PRFSL) HACK. in DC. Monog. Phan. 6 (1889) 233; MERR. 1. c. 51.

Isachaemum polystachyum PRLSL, Rel. Haenk. 1 (1830) 328.

The variety collected in Guam. Palau, Korror 153. The species in Yap (Vorkens). Distrib. The Philippines to the Moluccas and New Hanover (species).

Ischaemum longisetum Merr. 1. c. 52.

Guam (MerrilL). Distrib. The type from Guam, endemic.

Ischaemum muticum LINN. Sp. Pl. (1753) 1049; Volkens 1. c. 456. Yap (Volkens). Distrib. India through Malaya to tropical Australia, New Caledonia and western Polynesia.

Ischaemum rugosum SALISB. Ic. (1791) 1, t. 1; Merr. 1. c. 51. Guam (Merrill). Distrib. India to Malaya.

\section{Manisuris SwAKTZ}

Manisuris granularis LinN. f. Nov. Gram. Gen. (1779) 37, f. 47; Vol.KENS 1. c. 456.

Yap (Volkens). Distrib. Pantropic.

\section{Miscanthus ANDERSSON}

Miscanthus floridulus (LABILl.) Warb. ex K. Schum. \& LAUTERB. Fl. Deutsch. Schutzg. Südsee (1901) 166; Merr. 1. c. 51. 
Sacharum floridulum Labil,.. Sert. Austr. Galed. (1824) 13, t. 18. Guam; Pagan 2198. Distrib. Formosa to Australia and Polynesia.

Monerma Beauvors

Monerma repens (Forst.) Beauv. Agrost. (1812) 117; MERr. l. c. 58.

Rotthoellia repens Fonst. Prodr. (1797) 9 ; KoIDz. in Bot. Mag. Tokyo 29 (1915) 247.

Jaluit, Guam. Along the seashore. Distrib. Ceylon to Australia and Polynesia.

\section{Oplismenus Beauvors}

Oplismenus compositus (Linn.) BEAuv. Agrost. (1812) 54 ; Volkens. l. c. 456 ; Merr. l. c. 56 .

Panicum compositum LinN. Sp. Pl. (1753) 57.

Yap; Guam. Distrib. Pantropic.

\section{Oryza LinNaEus}

Oryza sativa LiNN. Sp. Pl. (1753) 333 ; VolkfNs 1. c. 456; MFrr. 1. c. 56 .

The rice. Cultivated in the warm parts of both hemispheres.

\section{Panicum Linnaeus}

Panicum ambiguum Trin. in Mém. Acad. St. Pétersb. VI 3, 2 (1835) 243 ; Volkens 1. c. 456 ; Merr. 1. c. 55.

Guam (Merrill), Yap (Volkens). Distrib. Burma to Mascarene Islands, Malaya and Polynesia.

Panicum colonum Linn. Syst. ed. i0 (1759) 870; Volkens 1. c. 456 ; MERR. 1. c. 55.

Yap (Volkens), Guam (Mrrril.'). Distrib. Pantropic.

Panicum distachyum LiNN. Mant. 1 (1767) 183; MerR. 1. c. 55. Guam. Distrib. India to Malaya and Polynesia.

Panicum crus-galli Link. Sp. Pl. (1753) 56; Volkens 1. c. 456. Yap (Volkens). Distrib. Pantropic.

Panicum indicum Linn. Mant. 1 (1767) 184; Volkens 1. c. 456. Yap (Volkens). Distrib. India through Malaya to tropical Australia.

Panicum isachne Roth, Nov. Pl. Sp. (1821) 54 ; Merk. 1. c. 55. 
Guam (introduced). Distrib. Meditaranean region to India.

Panicum luzoniense Prest, Rel. Haenk. 1 (1830) 303; Mrrk. 1. c. 55.

Guam (Merrill). Distrib. Malay Peninsula and the Philippines.

Panicum patens Linn. Sp. Pl. (1763) 18: Merr. Enum. Philip. Fl. P1. 1 (1922) 66.

Palau 322. Common in thickets and old clearings at low altitudes. Distrib. India to Malaya.

Panicum repens Linv. Sp. PI. ed. 2 (1762) 27.

Saipan 1022. Distrib. Pantropic.

Panicum reptans LinN. Syst. ed. 10 (1759) 871.

Panicum prostratum Lam. Ill. 1 (1791) 171; Vorkess 1. c. 456.

Yap. Distrib. Pantropic.

\section{Paspalum Linnafus}

Paspalum conjugatum BerG. in Act. Helvet. 7 (1772) 129, t. 8 ; Volkens 1. c. 456 ; Merk. 1. c. 53.

Saipan 952, Truk 604, Guam, Yap. Distrib. A native of tropical America, now pantropic.

Paspalum distichum L.nN. Amoen. Acad. 5 (1759) 391 ; VoLkens 1. c. 456.

Yap (Volkens). Distrib. Tropical and subtropical regions.

Paspalum scrobiculatum LinN. Mant. 1 (1767) 29; Volkens l. c. 53.

Palau 200, Yap (Volkens), Guam (Mrerrill). Distrib. Pantropic.

\section{Phragmites TRIniUs}

Phragmites karka (Ret7.) TRLN. ex. Steud. Nomen. 2 (1840) 324. Phragmites Roxburghii Steub. Nom. ed. 2, 2 (1841) 324, et Syn. Glum. 1 (1855) 196; Volkens I. c. 457. Yap (VolkE.NS). Distrib. India to Malaya.

Phragmites vulgaris (LAM.) Trin. Fund. Agrost. (1820) 134. Anudo vilgaris LaM. Fl. Franc. 3 (1778) 615.

Palau, Korror 156. In thickets. Distrib. All warm countries.

Pollinia TRINIUS 
Pollinia glabrata (BRongn.) Trin. in Bull. Acad. Pétersb. 1 (1836) 70 ; MerR. 1. c. 51.

Eulalia glabrata Brongn. Bot. Duperry's Voy. (1829) 93, t. 19.

Guam (Merrjul). Distrib. Otherwise known from New Caledonia and the Society Islands.

\section{Rottoboellia Linnaeus $f$.}

Rottoboellia sp. VoLKens 1. c. 456.

Yap.

\section{Saccharum LinNaEUS}

Saccharum officinalum LiNN. Sp. Pl. (1753) 54; Volkens l. c. 455 ; Merr. 1. c. 51.

Probably a native of south-eastern Asia, now cultivated in all tropical countries. Sugar cane.

\section{Schizostachyum NeES}

Schizostachyum lima (Branco) Merr. in Am. Journ. Bot. (1916) 62 ; KANEHIRA in Bot. Mag. 45 (1931) 273.

Bambusa lima BLANCO Fl. Filip. (1837) 271.

Oxythenanthera Warburgii (HACK.) (non K. Schum.) VOLKeNs 1. c. 457.

Palau: Korror 168, 181, Kamset 529, Garamiscan 542, 2111; Yap 1104. A remarkable bamboo having unusually long internodes. Distrib. Philippines. Nat. name: "arirudo" (Palau), "poo" (Yap).

\section{Setaria Beauvors}

Setaria flava (Nees) Kunth Rev. Gram. 1 (1929) 46; Merr. l. c. 56.

Panicum flavum NeEs ex Trin. Gram. Panic. (1826) 162.

Guam (MERriLL). Distrib. Warmer parts of both hemispheres.

Setaria glauca (Linn.) P. BeAuv. Agrost. (1812) 51 ; Volkens 1. c. 456.

Yap (Volkens). Distrib. Pantropic. 
Sporobolus indicus (LinN.) R. BR. Prodr. (1810) 170 ; MeRR. I. c. 56.

Agrostis indica LinN. Sp. Pl. (1753) 63.

Guam (Merrill). Distrib. Pantropic.

Sporobolus virginicus (Linn.) Kunth Rev. Gram. 1 (1829) 67; MERR. 1. c. 57.

Agrostis virginicus LINN. Sp. Pl. (1753) 63.

Guam. Distrib. Pantropic. Common near the seashore.

\section{Stenotaphrum TRINIUS}

Stenotaphrum subulatum TRIN. in Mém. Acad. Pétersb. VI, Sci. Nat. 3 (1835) 190 ; MerR. l. c. 56.

Guam (MerriLL). Distrib. Mascarene Islands to New Caledonia and the islands off the north east coast of Australia.

\section{Syntherisma WALTER}

Syntherisma corymbosa (RoxB.) Hosokawa in Trans. Nat. Hist. Soc. Formos. 24 (1934) 199.

Panicum corymbosum RoxB. Hort. Beng. (1814) 7, nom. nud. Fl. Ind. 1 (1820) 292.

Digitaria corymbosa Merr. Enum. Philip. Fl. PI. 1 (1923) 53.

Saipan, Palau (Hosokawa). Distrib. India to Southern China and Formosa through Malaya to Samoa.

Syntherisma longiflora Skefls in U. S. Department Agr. Buil. 261 (1912) 30; HosoKawa 1. c. 200.

Ponape, Parkier HosoKawa 5875. Distrib. Warmer parts of the Old World

Syntherima mariannensis HosoKawA 1. c. 198.

Digitaria mariannensis MerR. 1. c. 54.

Guam (Merrill). Distrib. Endemic.

Syntherisma Mezii HosoKawa. 1. c. 198.

Digitaria mariannensis Møz in ENGL. Bot. Jahrb. 59 (1924) 1, non Merr. in Philip. Journ. Sci. 9 (1914) Bot. 54.

Digitaria Mezii KanehIRA Fl. Micr. (1933) 401.

Saipan GibBon 1179. Distrib. Endemic. 
Syntherisma Robinsonii Hosokawa 1. c. 198.

Digitaria Robinsonii MERR. in Philip. Journ. Sci. 15 (1915) Bot. 540. Guam (Merrill). Distrib. Endemic.

Syntherisma sanguinalis Dulac. Fl. Hautes Pyr. (1867) 77.

Panicum sanguinale LiNn. Sp. Pl. (1753) 57; VolKens 1. c. 456; KoIDz. in Bot. Mag. Tokyo 29 (1915) 246.

Digiataria sanguinalis (LiNN.) Scop. Fl. Carn. ed. 2, 1 (1772) 52; MERR. 1. c. 54.

Yap (Volkens). Jaluit (Koldzlimi). Distrib. All warm countries.

Syntherisma stricta Hosokawa 1. c. 198.

Digilaria stricta Gaudich. Bot. Freyc. Voy. (1826) 409 ; Merr. 1. c. 59.

Panicum Gaudichaudii Kunth Rev. Gram. (1830) 385, t. 106.

Guam (Merrill). Distrib. Endemic.

\section{Thuarea PERSOON}

Thuarea involuta (Forst. f.) R. \& S. Syst. 2 (1817) 872 ; Merr. in philip. Journ. Sci. 15 (1919) Bot. 540.

Ischaemum involutum Forst. f. Prodr. (1786) 73.

Thuarea saramentosa PERS. Syn. 1 (1805) 110.

Guam (Merrili). Distrib. A common Malay-Polynesian strand plant. Jap. name: Sunazasa.

\section{Zea LINNAEUS}

Zea Mays Linn. Sp. Pl. (1753) 971; Volkens 1. c. 50; Merr. 1. c. 50.

Ponape 1597, Yap; Guam. Jap. name: Tômorokosi. Distrib. Introduced from America, now widely planted.

\section{Zoisia WILLDENOW}

Zoisia matrella (LinN.) MERr. in Philip. Journ. Sci. 7 (1912) Bot. 230 .

Agrostis matrella LinN. Mant. 2 (1771) 185.

Zoisia pungens WILD. in Ges. Naturf. Fr. Neue Schrift 3 (1801) 441; VOLKENS 1. c. 456.

Yap (Volkens). Distrib. Tropical Asia and Africa through Malaya to Australia and west Polynesia. 


\section{Cyperaceae}

\section{Carex Linnaeus}

Carex fuirenoides Gaudich. Bot. Freyc, Voy. (1826) 412; Merk. l. c. 58; KüKenth. in ENGL. Bot. Jahrb. 59 (1924) 10. Guam (Merrili). Distrib. Endemic.

\section{Cladium P. BRowNE}

Cladium aromaticum Merr. in Philip. Journ. Sci. 9 (1914) Bot. 59;

KÜKENTH. 1. c. 7.

Guam (Mrrili). Distrib. Philippines.

Cladium colpodes Lauterb. in Schum. et Lauterb. Nachtr. Fl.

Deutsch. Schutzg. Südsee (1905) 59; KüKENTH. 1. c. 7.

Palau. Distrib. New Guinea.

Cladium Gaudichaudii W. F. WIGIT ex SAFroRd in Contr. U.S. Herb. 9 (1905) 230 ; MerR. 1. c. 59 ; KüKeNTir. 1. c. 7.

Guam (Merrill); Palau, Aimiriik 1967, 2345; Yap 1236. Distrib. Endemic.

\section{Cyperus Linnaeus}

Cyperus compressus Linn. Sp. PI. (1753) 46; MlerR. 1. c. 59; KüKLNTH. 1. c. 3.

Guam (Merrill), Ponape, Jaluit. Distrib. Tropics.

Cyperus eyperoides (LINN.) KÜKENTH. subsp. cyperinus (VAHL) KÜKENTH. 1. c. 3.

Cyperus cyperinus VAHL ex VolKens 1. c. 457.

Yap, Palau, Ponape; Tinian 1057. Distrib. Tropical Asia to Polynesia.

Cyperus difformis Linn. Cent. Pl. 2 (1756); MerR. 1. c. 59 ; KïKENTH. 1. c. 3 .

Guam (MERRLL). Distrib. All tropical Countries.

Cyperus ferax var. Novae-Hannoverae (BоFск.) K̈̈KENTH. 1. c. 4. Cyperus Novae-Hannoverae BoEck. in ENGI. 1. c. 5 (1884) 91.

Marianne Islands, Palau, Ponape. Distrib. New Guinea.

Cyperus Haspan Linn. Sp. Pl. (1753) 45; Volkens 1. c. 457; KüKENTH. 1. c. 3. 
Yap (Volkens), Palau. Distrib. Pantropic.

Cyperus polystachyus RotTB. Desc. Nov. Pl. (1773) 39 ; KüKenTh 1. c. 3.

Marianne Is. (Gaudich $\Lambda$ ud) ; Ponape. Distrib. Pantropic.

Cyperus rotundus LinN. Sp. Pl. (1753) 45; Volkenss 1. c. 457;

MerR. 1. c. 59 ; KüKenth. 1. c. 3.

Yap (Volkens). Jaluit. Distrib. Pantropic.

Cyperus Zollingeri Steud. in Zoll. Verz. Ind. Archipel. (1854) 62 ; VOLKENS 1. c. 457 ; KÜKENTH. 1. c. 3.

Yap (VolKens), Truk. Distrib. Tropical Asia and Africa and through Malaya, Philippines to tropical Australia.

\section{Diplacrum R. BRown}

Diplacrum carcinum R. BR. Prodr. (1810) 241; Merr. 1. c. 60; KÜKENTH 1. c. 9.

Scleria carcina Benth; Volkens 1. c. 458.

Guam, Palau, Yap. Distrib. India to China, Philippines southward to Australia.

Eleocharis R. BRowN

Elcocharis atropurpurea KUNTH, Enum. PI. 2 (1837) 151 ; KÜKENTI. 1. c. 4 .

Eleocharis capitata (non R. Br.) Merr. 1. c. 60.

Guam (Merrill). Distrib. Tempcrate and tropical zonc.

Eleocharis dulcis (Burm. f.) TRIN. ex Henschel Vita Rumph. (1833) 186; MerR. Enum. Philip. Fl. Pl. 1 (1922) 119.

Andropogon dulcis BuRm. f. Fl. Ind. (1768) 219.

Palau: Marikyoku 444. An aquatic plant. Distrib. India to Malaya.

Eleocharis plantaginoides (ROTTB.) W. F. WIGHT in Contr. U.S. Nat. Herb. 9 (1905) 268; Merr. 1. c. 60; KüKenth. I. c. 4.

Scirpus plantaginoides Rotri. Descr. et Icon. Pl. (1773) 45, t. 16 , f. 2.

Guam (MerridL), Palau. Distrib. Widely distributed in the tropics of the Old World.

Eleocharis variegata PRESL var. laxiflora (Thw.) C. B. ClakKe in Hook. f. Fl. Brit. Ind. 6 (1893) 626; KüKENTII. 1. c. 4. 
Eleocharis fistulosa (non Schult.) Volkens 1. c. 458.

Ponape 679, 1515; Yap 1152. In open wet places. Distrib. India to Madagascar, China, Malaya and Polynesia.

\section{Fimbristylis VAHL}

Fimbristylis annua (ALL.) R. et S. Syst. 2 (1817) 95, var. diphylla (RETZ.) KËKENTH. I. c. 4.

Guam, Truk; Saligan 2166; Yap 1243, 1099; Palau 2120; Ponape 680. Distrib. Endemic.

forma tomentosa KüKENTH. 1. c. 4.

Guam, Saipan; Ponape 680; Palau 1; Yap 1099, 1243. A weed along roads. Distrib. Endemic.

var. Royeniana (NEES) KüKLNTII. forma exolicata KüKLENTH. 1. c. 5. Palau, Yap. Distrib. Endemic.

var, podocarpa (Ners et Meyen) KüKenth. 1. c. 5.

Ponape. Distrib.

var. pseudoferruginea KёKENTH. 1. c. 5.

Ponape, Palau. Distrib.

Fimbristylis autumnalis (LINN.) R. et S. var. complanata (RETZ.) KÜKENTH. 1. c. 6.

Yap. Distrib.

forma hemispherica KüKENTH. 1. c. 6.

Guam. Distrib.

Fimbristylis cymosa R. BR. Prodr. (1810) 228.

Saipan 1010. Distrib. Polynesia.

Fimbristylis cymosa R. BR. var. umbellato-capitata (MANN.) HiLLEBR. Fl. Haw. Is1. (1888) 473 ; KüKFenth. 1. c. 6.

Guam. Distrib. Sandwich Islands.

Fimbristylis globulosa (ReTz.) KunTh, Enum. 2 (1837) 231; VoIKENS 1. c. 458; MirR. 1. c. 61; KÜKRENTII. 1. c. 5.

Scirpus globulosa RETz. Obs. 6 (1791) 19.

Guam, Yap. Distrib. India to Malaya. 
Fimbristylis marianna GaUdich, in Bot. Freyc. Voy. (1826) 413; K̈̈IKENTH. 1. c. 5.

Fimbristylis maxima K. Schym. Fl. Kais. Wilh.-Land (1889) 24;

VolKens 1. c. 458 ; MerR. 1. c. 61.

Guam; Yap 1148; Palau 117. Distrib. Endemic.

Fimbristylis miliacea (LINN.) V $\Lambda$ HL, Enum. 2 (1806) 287 ; Merr.

1. c. 61 ; KüKENTH. 1. c. 6 .

Scirpus miliaceus LinN. Syst. ed. 10 (1759) 868.

Ponape, Kolonia 678. In waste places. Distrib. Pantropic.

Fimbrisylis nutans VAHL, Enum. 2 (1806) 285; Volkens 1. c. 452; KÜKENTH. I. c. 4.

Yap. Distrib. Tropical Australia and Asia.

Fimbristylis pauciflora R. BR. Prodr. (1810) 225; Kïkenth. 1. c. 4. Eleocharis acicularis (non R. Br.) VolkENs 1. c. 458.

Yap. Distrib. Tropical Asia and Australia.

Fimbristylis spathacea RoTH, Nov. Sp. Pl. (1821) 24 ; VoLKENS 1. c. 458; KoIDz. in Bot. Mag. Tokyo 29 (1915) 247.

Yap, Jaluit; Rota 1821; Palau 2028; Anatahan 2150.

Fimbristylis urakasiana KǗkENTII. 1. c. 5.

Urakas Island (Marinnae Islands). Distrib. Endemic.

\section{Fuirena RottBonLL}

Fuirena umbellata RoTTs. Descr. Ic. (1773) 70, t. 19, 1. 3; VoLKENS 1. c. 457 ; Merr. 1 c. 62 ; Kükenth. 1. c. 6.

Ponape, Kolonia 681; Yap 1147, 1149, 1246; Guam, Palau. Distrib. Pantropic.

Hypolytrum L. C. RrCHARD

Hypolytrum dissiflorum S'reud. Syn. Pl. Cyp. 132 ; K̈̈kenTH. 1. c. 7. Ponape 630, 807; Kusai 1341. Distrib. The Caroline and Marshall Islands.

var. oligostachyum Schum. et LAuterb, Fl. Deutsch. Schutzg. Südsee (1901) 190.

Ponape. Distrib. The type from Ponape; endemic.

Hypolytrum latifolium L. C. RICH. in PERS. Syn. 1 (1805) 70; VOLKENS 1. c. 457 ; KëKENTH. 1. c. 7. 
Yap 1140. Distrib. India to Formosa, Philippines, Malaya to Polynesia. Jap. name : Suge-gaya.

\section{Kyllingia RotTBOELL}

Kyllingia brevifolia RotTr. Descr. Ic. (1773) 13, t. 4, f. 3 ; Merr. 1. c. 62 ; KüKENTH. 1. c. 2.

Palau, Marikyoku 399; Ponape, Kolonia 733; Tinian 1056; Guam. Distrib. Pantropic.

Kyllinga monocephalia RotTB. Descr. Ic. (1773) 13 , t. 4, f. 4 ; Volkens 1. c. 457 ; Merr. 1. c. 62 ; KÜKenth. 1. c. 2.

Saipan 902 ; Palau, Ponape, Yap and Guam; Anatahan 2144. Dis. trib. Pantropic. Jap. name: Oohime-kugu.

Lepironia L. C. RiciIARD

Lepironia mucronata L. C. Rich. in Pers. Syn. 1 (1805) 70; Volkens 1. c. 458 ; KüKENTH. 1. c. 9.

Yap. (Volkens). Distrib. Madagascar, Australia and Polynesia.

\section{Mariscus GALRTNER}

Mariscus cyperinus (RET7.) VAHL, Enum. 2 (1806) 377 ; NEES in Hook. Journ. Bot. Kew Micel. 6 (1854) 28.

Kyllinga cyperinus Retz. Obs. 6 (1791) 21.

Tinian 1057. In thickets. Distrib. India to Malaya and Polynesia. Jap. name: Taiwan-kugu.

Mariscus pennatus (LAM.) Merr. Enum. Philip. Fl. Pl. 1 (1922) 113. Cypenus pennatus LaM. Tab. Encycl. 1 (1791) 114.

Mariscus albescens Gaudich. Bot. Freyc. Voy. (1826) 415; KoIDz. in Bot. Mag. Tokyo 29 (1915) 247.

Cyperus stuppeus Forst. f. Prodr. (1786) 89, nom. nud.; KüKEnTH. 1. c. 3 .

Mariscus stuppeus Merr. in Philip. Journ. Sci. 3 (1908) Bot. 398, 9 (1914) Bot. 62.

Kusai 1307; Anatahan 2153; Guam; Jaluit; Yap 68, Palau 391; Ponape. Along shores and in mangrove swamps. Distrib. Tropical Africa to Southern Asia, Malaya and Polynesia. Jap. name: Onikugu.

\section{Rynchospora VAHL}

Rhynchospora corymbosa (LiNN.) BRITY. in Trans. N. Y. Acad. Sci. 11 (1892) 84; MerR. 1. c. 62 ; KüKenTTI. 1. c. 6. 
Scirpus corymbosus LinN. Cent. PI. 2 (1756) 7.

Rhyncospora aurea VAHL, Volkens 1. c. 458.

Guam, Yap, Palau; Truk 607; Ponape 1513. In swampy places. Distrib. Widely distributed in the tropics of both hemispheres.

Rhynchospora rubra (Lour.) Makino in Bot. Mag. 'Tokyo 17 (1903) 180 ; Merr. 1. c. 62 ; KüKenth. 1. c. 6.

Schoenus nubra Lour. Fl. Cochinch. (1780) 41.

Rhyncopspora Wallichiana KunTH, Enum. 2 (1837) 289; VolKens 1. c. 458 .

Yap 1164; Palau 115, 202; Guam. Distrib. Tropical Africa and Asia to Japan, southward through Malaya to Australia. Jap. name:

\section{Schoenus LinNAEUS}

Schoenus calostachyus (R. BR.) POIRIY' ex KïKenth. in Engl. Bot. Jahrb. 59 (1924) 7.

Schoenus triangularis VoLKENS 1. c. 458.

Yap, Palau (Lemermann). Distrib.

\section{Scirpodendron ZIPPEL}

Scirpodendron ghaeri (GNERTN.) MERR. in Philip. Journ. Sci. 9 (1914) Bot. 269.

Chionanthus ghaeri GAERTN. Fruct. 1 (1781) 190, t. 29, f. a-c.

Scirpodendron costatum KuRz in Jonrn. As. Soc. Beng. 39, 2 (1869) 85; VOLKENS I. c. 458 ; KüKRNTII. 1. c. 9.

Yap; Ponape 1685. In wet places near the seashore, also along streams at low altitudes.

Distrib. Ceylon through Malaya to Australia, Philippines and Polynesia.

\section{Scirpus LinnaEUS}

Scirpus erectus Porr, in LAM. Encycl. 6 (1804) 671; MerR. 1. c. 63; KÜKENTH. 1. c. 6.

Guam. Distrib. Warmer parts of both hemispheres.

Scirpus littoralis ScırRAD. Fl. Germ. 1, 142, t. 5, f. 7 ; Kükenth. 1. c. 6 .

Saipan (Höfer). Distrib. Europe, Asia and Aegypt. 


\section{Scleria BERGius}

Scleria bancana Miq. Fl. Ind. Bat. Suppl. (1862) 693; KüKENTH. 1. c. 9 .

Palau, Korror (Ledermann). Distrib. Tenasserium through Philippines, Malaya to New Guinea.

Scleria lithosperma (Linn.) Sw. Prodr. (1788) 18; Volkens 1. c. 458 ; Kükenth. 1. c. 9; Merk. in Philip. Journ. Sci. 15 (1915) 541.

Scirpus lithospermus LinN. Sp. P1. (1758) 51.

Saipan 1028; Yap 1118; Aramagan 2189. Distrib. Pantropic. Jap. name: Takao-sinzyugaya.

Scleria Margaritifera Willd. Sp. Pl. 4 (1805) 312; Volkens 1. c. 458 ; MrRr. 1. c. 63 ; Kükfenthl 1. c. 9.

Scleria hebecarpa (non NEES) VolkENs 1. c. 458.

Palau 118; Ponape 1567; Yap 1111. In open field. Distrib. Polynesia and Australia.

Scleria Merrillii Palda in Atcem. Bot. Zeitschr. 17 (1911) Beil. 8; KüKENTH. 1. c. 10.

? Scleria laxa R. Br. Prodr. (1810) 240; Merr. 1. c. 63.

Guam, Ponape. Distrib. Philippines.

Scleria multifoliata ВOFCK. in Linnaea 38 (1874) 510; KÜKENTH. 1. c. 9 .

Palau, Babeldaob; Ponape, Leperei. Distrib. Malaya.

Scleria scrobiculata NELS in WIGHT, Contr. (1834) 117; KüKENTII. 1. c. 9 .

Palau: Korror and Babeldaob. Distrib. Andamann Islands, Philippine, Riu-kiu and New Guinea. Jap. name: Oo-sinzyugaya.

Scleria zeylanica PoIR. in Encycl. 7 (1806) 3; KüKeNTr. 1. c. 10. Palau, Babeldaob. Distrib. Malaya.

\section{Thoracostachyum KURZ}

Thoracostachyum hypolitroides C. B. C.ARKE in HoOK. f. Brit. Ind. 6 (1894) 680; KüKENTH. 1. c. 8.

Palau, Babeldaob. Distrib. Asia and tropical Australia.

var. immensum KüKENTH. 1. c. 9. 
Ponape: Leprerei (LedermanN); Ponape 1673. In swampy places at medium altitudes.

Nat. name: "pohge" (Ponape).

\section{Torulinium Desvaux}

Torulinium ferax (L. C. Rich.) HAM. Prodr. Pl. Ind. Occ. (1825) 15. Cyperus ferax L. C. Rich. in Act. Soc. Hist. Nat. Paris 1 (1792) 106. Ponape 851; Guam (Merrill). Distrib. Patropic.

\section{Palmae \\ Areca LinNaEUS}

Areca Catechu Linn. Sp. Pl. (1753) İl89; KanehIRA in Bot. Mag. Tokyo 45 (1931) 274.

Palau, Ponape. Planted. Distrib. Widely distributed in the IndoMalayan region, cultivated in Palau, I'onape etc. The areca nut. Fruit is used by the natives for chewing with betle leaves.

\section{Cocos IJNAAELS}

Cocos nucifera LINN. Sp. Pl. (1753) 1188; KANimIIRA in Bot. Mag. Tokyo 45 (1931) 274.

The coco-nut palm. Distrib. Pantropic. Nat. names: "nius" (Palau), " niu" (Yap), "nu" (Truk), "ni" (Ponape), "nu" (Kusai), "nizok" (Rota, Saipan).

\section{Coelococcus H. WENDLAND}

Coelococcus amicarum (WENDI.) W. F. WIGHT ex SAFFORD in Contr. U. S. Herb. 9 (1905) 244; KanemrRA in Bot. Mag. Tokyo 45 (1931) 274.

Sagus amicainm WendL. in Bot. Zeit. 36 (1878) 115.

Coelococcus carolinensis Dingl. in Bot. Centralb. 32 (1887) 349.

Ponape 879 . In primary forests at low altitudes. It occurs also in Truk and Kusai, occasionally planted in Ponape. Distrib. Fndemic. Nat. name: " hibun" or "rubun" (Truk). The ivory nut.

\section{Elaeis JACQUIN}

Elaeis guineensis JACQ. Select. Am. (1763) 280, t. 172.

Planted in Ponape. A native of tropical America. The oil palm. 


\section{Exorrhiza BeCCARI}

Exorrhiza carolinensis (Becc.) BURRET, Fedde Repert. 24 (1926) 296 ; Kanehira in Bot. Mag. Tokyo 48 (1934) 731.

Bentinckiopsis carolinensis BEcc. in Webbia 5, 1 (1921) 113, ENGL. Bot. Jahrb. 59 (1924) 16; KANeHIRA in Bot. Mag. Tokyo 45 (1931) 274.

Cyphonokentia (?) carolinensis BECC. in ENGL. 1. c. 52 (1915) 4.

Truk (KRÄMER). Distrib. Endemic. Nat. name: "kiriyau" (Truk).

Exorrhiza ponapensis (BEcc.) BuRret 1. c. 394 ; KaneHIRA 1. c. 732. Bentinckiopsis ponapensis BEcc. in Webbia 5, 1 (1921) 113; KANEHIRA, Fl. Micr. (1933) 72, f. 9.

Ponape 770,1675 . A very tall palm, scattered in primary forests at medium altitudes. Distrib. Endemic. Nat. name: "kotop" (Ponape) ; Jap. name: "otoko-yasi."

\section{Glubiopsis BECCARI}

Glubiopsis palauensis BFCC. in ENGL. Bot. Jahrb. 59 (1924) 11 ; KANEHIRA in 13ot. Mag. Tokyo 45 (1931) 274, Fl. Micr. 76, f. 11. Palau 114, 1952, 3442. Distrib. Endemic. Nat. name: "akaboek" (Palau).

\section{Heterospathe SCHEFFER}

Heterospathe palauensis BECC. in ENGL. Bot. Jahrb. 52 (1914) 4; Kanitira Fl. Micr. 76, f. 12.

Palau 1S04. Distrib. Endemic. Nat. name: "asbó" (Palau).

\section{Nypa Wurmb}

Nypa fruticans Wurmb in Verh. Bat. Genoots 1 (1779) 350; KanEHIRA in Bot. Mag. Tokyo 45 (1931) 273.

Palau 451. Along tidal streams in brackish swamps. Distrib. India through Malaya to tropical Australia. Nat. names: "aian" (Yap), "kuwa". (Truk), "fals" (Kusai), " toyel" (Palau).

\section{Ponapea BECCARI}

Ponapea Ledermannii BECC. in ENGL. Bot. Jahrb. 59 (1924) 14; KANEHIRA 1. c. 274, Fl. Micr. 78, f. 13.

Ponape 742 ; Kusai 1361 . In primary forests at low and medium altitudes. Distrib. Endemic. Nat. name: "kattai" 


\section{Araceae}

\section{Alocasia Necker}

Alocasia indica (RoXi.) SchotT, VolkeNs 1. c. 459; KoIDz. in Bot. Mag. Tokyo 29 (1915) 247; ENGL. in DC. Monog. Phan. 2 (1879) 501.

Yap (Volkens), Jaluit (KoIDzumi). Distrib. Tropical Asia.

Alocasia macrorrhiza (Linn.) Schotт, Melet 1 (1832) 18; ENGL.

1. c. 502 ; MERR. 1. c. 64 .

Arum macrorrhiza LinN. Sp. P1. (1753) 965.

Saipan 941. Distrib. India to Malaya, planted for ornamented purposes.

\section{Colocasia Sснотт}

Colocasia esculenta (LinN.) Schott Melet 1 (1832) 18; MERR. 1. c. 64 .

Arum esculentum Lins. Sp. Pl. (1753) 965.

Colocasia antiquorum SCHOTT 1. c. 18; VolkENs 1. c. 459.

Palau 449, 450; Yap (Volkens). Distrib. Pantropic in cultivation. This is "Taro."

\section{Cyrtosperma Griffith}

Cyrtosperma Chamissonis (SchotT) Merr. 1. c. 65.

Arisacontis Chamissonis ScHoTT in Bonplandia 5 (1857) 129.

Cyrtosperma edule SchotT ibid. 9 (1861) 267, ENGs. Pflanzenr. 48 (1911) 17; VoLKENS 1. c. 459.

Guam, Yap (Volkens). Distrib. New Guinea to Fiji, in cultivation.

\section{Epipremnum SchotT}

Epipremnum carolinensis Volkens 1. c. 459. Yap (Volkens). Distrib. Endemic.

Epipremnum pinnatum (LiNn.) ENGL. Pflanzenr. 37 (1908) 60. Pothos pinnata Linx. Sp. Pl. ed. 2 (1763) 1374.

Palau 390, 393. An epiphyte on a tree along roads. Distrib. Tenasserium to New Guinea and Polynesia.

\section{Homalomena SсHOTT}

Homalomena rubescens KunTh, Enum. Pl. 3, 57; Volkens 1. c. 459. 
Yap (VolKens). Distrib. East Indies.

Rhaphidophora HASSKARL

Rhaphidophora Engleri (ENGl. et KraUse) KaneHIRA nom. nov. Rhaphidophora palauensis ENGL. et Krause in ENGL. Bot. Jahrb. 56 (1921) 433; non KoIDz. in Bot. Mag. Tokyo 30 (1916) 400. Palau: 565, Babeldaob (Ledermann). Distrib. Endemic.

Rhaphidophora palauensis KoIDz. in Bot. Mag. Tokyo 30 (1916) 400.

Palau: Angaur (Komzumi \& Kaмrya). Distrib. Endemic.

Rhaphidophora Koidzumii (KoIDZ.) KANEHIRA nom. nov.

Epipremnum palauense KoIDz. in Bot. Mag. Tokyo 30 (1916) 401. Palau, Korror 165. The type from Korror. Distrib. Endemic.

\section{Flagellariaceae}

\section{Flagellaria LinNaEus}

Flagellaria indica LINN. Sp. Pl. (1753) 333; VolKENS 1. c. 453; Mkrk. 1. c. 65 ; Kanenira in Bot. Mag. Tokyo 45 (1931) 274. Palau: Aimiriik 257, Marikyoku 440; Ponape Kolonia 715; Saipan 899. A climber in forests from low to high altitudes. Distrib. Tropical Africa, tropical Asia through Malaya to tropical Australia.

\section{Hanguana BLuMF}

Hanguana aquatica KANEHIRA in Trans. Nat. Hist. Soc. Formos. 25 (1935) 8, f. 10.

Palau 398. In Galdok Pond. Distrib. Endemic.

\section{Xyridaceae}

\section{Xyris Linnafus}

Xyris pauciflora WiLld. Phytogr. (1794) 2, t. 1; Hosokawa in Trans. Nat. Hist. Soc. Formos. 24 (1934) 201.

Palau Korror (Hosokawa). Distrib. India to China, Philippines and Formosa through Malgya to Australia. Jap. name: Tôen-sô.

\section{Eriocaulaceae}

\section{Eriocaulon LinNaeus}


Eriocaulon longifolium NeFs et KunTH, Enum. P1. 3, 567; VoLKENS 1. c. 459.

Yap (VolKens). Distrib. Tropical Asia, Madagascar.

Eriocaulon sp.

Palau Marikyoku 443, 418. In open wet places.

\section{Bromeliaceae}

Ananas TOURNEFORT

Ananas comosus (LinN.) Merr. Interpret. Herb. Amb. (1917) 133, Sp. Blancoanae (1916) 94.

Bromelia comosus LiNN. Stickman Herb. Amb. (1754) 21. Ananas sativus Schultes f. Syst. 7, 2 (1830) 1283; Volkens 1. c. 459; Merr. 1. c. 65.

Distrib. A native tropical America, now cultivated in all tropical countries. Pineapple.

\section{Commelinaceae}

Aneilema R. BRown

Aneilema malabaricum (LINN.) MERR. in Philip. Jouan. Sci. 7 (1912) Bot. 232, 9 (1914) Bot. 66.

Tradescantia malabarica LiNN. Sp. Pl. ed. 2 (1762) 412.

Distrib. India to the Riu-kiu Islands southward to Malaya.

Aneilema vitiense Seem. F1. Vit. (1865-73) 314, t. S6, var. petiolata C. B. Clarke in DC. Monog. Phan. 3 (1881) 220; Merr. in Philip. Journ. Sci. 15 (1919) Bot. 542.

Guam (MerrilL). Distrib. Philippines, Moluccas to Polynesia.

\section{Commelina Plumier}

Commelina bengalensis LinN. Sp. Pl. (1753) 41; Merr. 1. c. 66.

Saipan 992; Guam (MERRILL). Distrib. Widely distributed in the tropics of the Old World.

Commelina nudiflora Linn. Sp. PI. (1753) 4; Volkens 1. c. 460; MerR. 1. c. 66.

Guam (Merrill), Yap (Volkens). Distrib. Pantropic.

Cyanotis D. DoN 
Cyanotis axillaris (LinN.) D. Don, Prodr. Fl. Nepal. (1825) 46; MERr. 1. c. 66 .

Commelina axillaris LinN. Sp. PI. (1753) 42.

Guam (McGregor). Distrib. India to China southward to tropical Australia.

Cyanotis crista (Linn.) D. Don, Prodr. Fl. Nepal. (1825) 46; MerR. 1. c. 66 .

Commelina crista LinN. Sp. PI. (1753) 42.

Guam. Distrib. Pantropic.

\section{Zebrina SCHNIZLEIN}

Zebrina pendula SchnIZL. in Bot. Zeit. 7 (1849) 870; Merr. 1. c. 66. Saipan 951, 2239. Distrib. Introduced from tropical America, now cultivated as an ornamental. Wandering jew.

\section{Liliaceae}

\section{Allium TOURNEFORT}

Allium cepa LnNo. Sp. Pl. (1753) 300; MFrR. 1. c. 66. Onion. Cultivated. Jap. name: Negi.

\section{Cordyline ROYEN ex ADANSON}

(Sanseviera THUNBERG)

Cordyline Roxburghiana (Schultes) Merr. in Enum. Philip. Fl. Pl. 1 (1922) 206.

Sanseviera Roxburghiana Schultes Syst. 7 (1829) 357.

Sanseviera zeylanica RoxB. Pl. Coromandel. 2 (1798) 43, t. 184, quod fig.; MrQ. Fl. Ind. Bat. 3 (1859) 559.

Cordyline hyacinthoides W. F. WIFHT in Contr. U. S. Nat. Herb. 9 (1905) 249, non Aloe hyacinthoudes LiNN.

Palau. Cultivated. Distrib. A native of tropical, Asia, now pantropic in cultivation. Bowstring hemp.

\section{Dianella LAMARCK}

Dianella ensifolia (LinN.) DC. in Red. Lil. (1802) t. 1; Volkens 1. c. 460 ; Merr. 1. c. 66 .

Dracaena ensifojia LinN. Mant. 1 (1767) 63.

Palau: Marikyoku 400, Aimiriik 1972; Guam; Yap. In forests at low altitudes. Distrib. India to the Mascarene Islands, China, Formosa, Malaya to tropical Australia and Polynesia. 


\section{Pleomele Salisbury}

Pleomene angustifolia (RoxB.) N. E. BR. in Kew Bull. (1914) 277 ; KaneHIRA in Bot. Mag. ToKyo 45 (1931) 275.

Dracaena angustifolia RoxB. Hort. Beng. (1814) 24.

Palau 511, 1990, 2449, 2772. In thickets and rocky'reefs. Distrib. India through Malaya to Australia. Nat. name: "orodakl" (Palau).

\section{Smilax TOURNEFoRT}

\section{Smilax sp.}

Truk 1723. In primary forests.

\section{Taetsia Medicus}

Taetsia fruticosa (LINN.) MerR. Interpret. Herb. Amb. (1917) 137. Convallaria fruticosa LiNN. Stickman Herb. Amb. (1754) 16.

Cordyline terminalis KunTH in Abh. Acad. Berl. (1820) 30; VolKENS 1. c. 460 ; MERR. 1. c. 67.

Yap (VolkeNs), Guam (MerRill). Distrib. Pantropic in cultivation, probably a native of Malaya. Jap. name: Sennen-boku.

\section{Amarilydaceae}

Ãgave Linnaeus

Agave viviparia LINN. Sp. Pl. (1753) 323; MerR. 1. c. 67.

Guam, Palau. Distrib. A native of Mexico, now widely distributed.

\section{Crinum Linnaeus}

Crinum asiaticum Linn. Sp. Pl. (1753) 292 ; Merr. 1. c. 67; KoIDZ. in Bot. Mag. Tokyo 29 (1915) 247.

Palau, Angaur 592; Saipan 1040; Kusai 1419; Jaluit; Guam. Distrib. India to Malaya and western Polynesia. Jap. name: Taiwan-hamaomoto.

Crinum macrantherum ENGL. in Bot. Jahrb. 7 (1886) 448; VoLKENS 1. c. 460.

Yap 1136. Distrib. Melanesia.

\section{Curculigo GaERTNer}

Curculigo orchioides Gaer'rN. Fruct. 1 (1788) 63, t. 13.

Guam ; Pagan 2211. In old fields. Distrib. India to Malaya. 


\section{Hymenocallis SaLisbury}

Hymenocallis littoralis (JACQ.) SALISB. in Trans. Hort. Soc. 1 (1812) 338.

Pancratium littorale JACQ. Select. Amer. (1763) 99.

Guam. Distrib. A native of Mexico, introduced.

\section{Hypoxis LinNaEuS}

Hypoxis aurea Lour. Fl. Cochinch. (1790) 200; Volkens 1. c. 460. Yap (Volkens). Distrib. India to Japan southward to the Philippines and Java.

\section{Polyanthes Linnaeus}

Polyanthes tuberosus LiNN. Sp. P1. (1753) 316.

Guam. Distrib. A native of tropical America, introduced. Jap. name: Geturaikô.

\section{Zephranthes HERBERT}

Zephranthes rosea LindL. Bot. Reg. 10 (1824) t. 821 ; MERr. 1. c. 68. Atomosco rosea GreEne Pittonia 3 (1897) 188.

Guam. Distrib. A native of tropical America.

\section{Taccaceae}

\section{Tacca Forster}

Tacca palmata Blume Enum. Pl. Jav. 1 (1827) 83.

Palau: Babeldaob 539, Aimiriik 292. Distrib. Malayan Archpelago.

Tacca pinnatifida Forst. Char. Gen. (1776) 70, t. 35; Volkens I. c. 460 ; MERR. 1. c. 68.

Palau 342 ; Saipan 1038; Yap 1164. Distrib. 'Tropical Africa and Asia through Malaya to Australia. Jap. name: Tasiro-imo. Nat. name: "mok-mok" (Truk, Ponape, Kusai), "gabgab" (Rota).

\section{Dioscoreaceae}

Dioscorea Plumier

Dioscorea alata LINN. Sp. Pl. (1753) 1033 ; MerR. l. c. 63.

Palau, Angaur 591. Distrib. Pantropic in cultivation, a native of the Old World Tropics. Jap. name: Daizyô. 
Dioscorea bulbifera LINN. Sp. Pl. (1753) 1033; MerR. I. c. 68. Palau Angaur 585; Kusai 1449. Distrib. India to China and Malaya. Jap. name: Kasyû-imo.

Dioscorea esculenta (Lour.) Burkill in Gard. Bull. Straits Settl. 1 (1917) 396; Merr. Interpret. Herb. Amb. (1917) 147.

Oncus esculentus Lour. Fl. Cochinch. (1790) 194.

Dioscorea aculeata (non LINN.) var. tiliaefolia PRAIN et BURKILL. apud MerR. 1. c. 68.

Saipan 1007, Guam (Merrilu). Distrib. India to Malaya and Polynesia.

Dioscorea korrensis R. KNUTH in ENGL, Pflanzenr. 87 (IV 43) 190. Palau, Korror. Distrib. Endemic.

Dioscorea Ledermannii R. KNUTH 1. c. 188.

Dioscorea flabellifolia (non PRAIN \& BuRKILL) KANEHIRA in Bot. Mag. Tokyo 49 (1935) 271.

Palau, Babeldaob 2316. In primary forests at about $100 \mathrm{~m}$ altitude. Distrib. Endemic.

Dioscorea palauensis R. KNUTH l. c. 191.

Palau, Korror. Distrib. Endemic.

Dioscorea pentaphylla LiNN. Sp. Pl. (1753) 1032.

Yap 1123, 1161. Distrib. India to Yunnan, southward through Malaya to New Guinea.

Dioscorea Raymundii R. KNUTH 1. c. 191.

Palau, Korror. Distrib. Endemic.

\section{Musaceae}

\section{Musa Linnaeus}

Musa sapientum Linn. Syst. ed. 10 (1759) 1393.

Widely cultivated. Banana. A number of forms are found in the islands.

Musa textilis NéE in Anal. Cienc. Nat. 4 (1801) 123.

Cultivated in Ponape, Yap, Kusai, Truk, etc. Abaca. Distrib. The Philippines. 
Ravenala madagascariensis GMeL. Syst. (1791) 567; K. Schum. in ENGL. Pflanzenr. 1 (1900) 29, f. 6 \& 7.

A native of Madagascar. Planted for ornamental purposes. Traveler's fan or Traveler's palm. Jaṕ, name: Oogi-basyô.

\section{Zingiberaceae}

Costus Linnaeus

Costus sericus Buume Enum. Pl. Jav. 1 (1827) 62.

Yap 1255; Palau: Korror 127, Aimiriik 323, Ogiwal 2064. In damp thickets at low altitudes. Distrib. Burma through Malaya, Philippines to New Guinea.

Costus speciosus (Koenig) Sm. in Trans. Linn. Soc. 1 (1791) 249; VOLKENS 1. c. 461.

Banksia speciosa KolNig in Retz. Obs. 3 (1783) 75.

Yap (Volkens). Distrib. Widely distributed in cultivation. Jap. name: Oo-hozaki-ayame.

\section{Curcuma Linnaeus}

Curcuma longa Linn. Sp. Pl. (1753) 2; Volkens 1. c. 460 ; Merr. 1. c. 69.

Yap (Vol.Kens), Guam (MerRILL). Distrib. A native of India, now pantropic. Turmeric. Jap. name: Ukon.

\section{Hedychium KoENIG}

Hedychium coronarium KoENIG in Retz. Obs. 3 (1783) 73.

Truk 615, Ponape 692. Distrib. A native of India, now pantropic, cultivated and naturalized.

\section{Languas KOENIG}

Languas babeldaobensis KaneHiRA in Trans. Nat. Hist. Soc. Formos. 25 (1935) 7. f. 9.

Palau $321 ! 430,2295$. In primary forests at low altitudes. Distrib. Endemic.

Languas carolinensis (Kowz.) KANEHIRA comb. nov.

Alpinia carolinensis KoIDz. in Bot. Mag. Tokyo 31 (1917) 233.

Ponape Kolonia 656, 1486. Distrib. The type from Ponape. Endemic. 
Languas pubiflora (BenTir.) Merr. Enum. Philip. Fl. PI. 1 (1922) 233. Hellenia pubiflora BeNTH. in HoOK. Lond. Journ. Bot. 2 (1843) 235. Alpinia pubiflora K. Schum. in ENGL. Pflanzenr. 20 (1904) 313; VOLKENS 1. c. 460.

Yap (Volkens). Distrib. New Guinea and Philippines.

Languas purpurata (VIEILI) KANEHIRA comb. nov.

Guillainia purpurata VIEILL in Bull. Soc. Linn. Norm. 10 (1866) 92. Alpinia purpurata (Viell.) K. Schum. 1. c. 323, f. 40, A ; Volkens 1. c. 460 .

Yap (Volkens): Distrib. New Hannover, Solomon Islands, Moluccas to New Caledonia.

\section{Zingiber ADANSON}

Zingiber officinale Rosc. in Trans. LINN. Soc. 8 (1807) 348; MERR. 1. c. 69 .

Guam. Distrib. A native of tropical Asia, now cultivated in all tropical and subtropical countries. Jap. name: Syôga.

Zingiber zerumbet (LrNN.) SM. Exot. Bot. 2 (1804) 103, t. 112; MerR. 1. c. 69.

Amomum zerumbet LiNN. Sp. Pl. (1753) 1.

Truk 616; Ponape Kolonia 685, 741; Yap 1128, 1209. Distrib. A native of tropical Asia, now patropic.

\section{Cannaceae}

\section{Canna Linnaeus}

Canna indica Linn. Sp. Pl. (1753) 1; Merk. 1. c. 69.

Guam (Mlerril.). Distrib. A native of tropical America, now pantropic. Jap. name: Dandoku.

\section{Marantaceae}

\section{Donax LOUREIRO}

Donax cannaeformis (Forst. f.) K. Schlm. in ENgrie. Bot. Jahrb. 15 (1893) 440.

Thalia cannaeformis Forst. f. Prodr. (1786) 1.

Palau: Korror 136, Aimiriik 269, 1934, 2351. Along streams and swampy places. Disirib. Java, Philippines, Formosa, Borneo to New Guinea. Jap. name: Kôtô-kumatakeran. 


\section{Maranta LinNaEUS}

Maranta arundinacea LINN. Sp. Pl. (1753) 2; MERR. I. c. 69.

Guam. Distrib. A native of tropical America, now widely distributed in cultivation. Jap. name: Kuzu-ukon.

\section{Burmanniaceae}

Burmannia LinnaEus

Burmannia coelestis D. Don, Prodr. Fl. Nepal. (1825) 44 ; Volkens I. c. 461.

Yap (Volkens). Distrib. India to Borneo and the Philippines.

\section{Orchidaceae}

\section{Acriopsis ReINWARDT}

Acriopsis javanica Reinw. ex BL. Cat. Gew Buitenz. (1823) 97; SCHLTR. in ENGL. Bot. Jahrb. 56 (1921) 491.

Palau: Aimiriik 290, Marikyoku 425, 1947, 2044. Distrib. Burma, Malaya, Philippines to New Guinea.

Acriopsis sp.

Garamiscan 563.

\section{Aglossorhyncha SCHLECHTER}

Aglossorphyncha micronesiaca ScHLTR. 1. c. 477.

Palau, Babeldaob. Distrib. Endemic. The genus distributed in New Guinea.

\section{Agrostophyllum BLuME}

Agrostophyllum palawense ScHLTR. l. c. 476.

The type from Palau, Babeldaob. Aimiriik 302, 1971, 2761. Distrib. Endemic.

Agrostophyllum nov. sp.?

Palau, Kamset 524, Aimiriik 1968. Distrib. Endemic?

Appendicula BuUme

Appendicula reflexa Blume Bijdr. (1825) 301; Schltr. 1. c. 478. Palau: Korror (RAymund), Marikyoku 426, Aimiriik 1946. Distrib. Java. 
Arundina Kanehirae Yamamoto in Trans. Nat. Hist. Soc. Formos. 23 (1933) 20.

Ponape 815,1610 . In forests at high altitudes. Distrib. Endemic.

Arundina sp. nov.?

Palau Aimiriik 2319.

\section{Bulbophyllum THOuars}

Bulbophyllum Gibbonianum ScHLTR. 1. c. 483.

The type from Babeldaob, Palau (Lfadermann), Aimiriik 299, 2328, Arumonogui-zan 517. Distrib. Endemic.

Bulbophyllum guamense Ames. in Philip. Journ. Sci. 9 (1914) Bot. 13; Merr. 1. c. 70 ; Schltr. 1. c. 482.

Guam. Distrib. Known only from Guam.

Bulbophyllum micronesiacum SchlTR. 1. c. 482.

Yap (Volkens); Ponape, Patapat and Paue. Distrib. Endemic.

Bulbophyllum ponapense SCHLTR. 1. c. 484.

Truk, Tol (not Ponape), Ledermann. Distrib. Known only from Tol Island, Truk. Endemic.

Bulbophyllum profusum Ames in Philip. Journ. Sci. 7 (1912) Bot. 128, 137 ; MfrR. 1. c. 70 ; SchlTR. 1. c. 485.

Guam. Distrib. The Philippines.

Bulbophyllum Volkensii SchlTR. in ENGL. Bot. Jahrb. 52 (1914) 11, 56 (1911) 485.

The type from Yap (Volkens), Palau (Krämer \& Ledermann). Yap 1263. Distrib. Endemic.

Calanthe R. BROWN

Calanthe triplicata (Wiliem.) AMes in Philip. Journ. Sci. 2 (1904)

Bot. 336; Merr. 1. c. 70; Schltr. ENGL. 1. c. 56 (1921) 479.

Orchis triplicata Willem. in Usteri Ann. Bot. 6, 18 (1796) 52.

Guam. Distrib. Widely distributed in the Indo-Malayan region.

\section{Ceratostylis BLume}

Ceratostylis sp. nov.?

Ponape, Kolonia 629! A genus not mentioned in the Schlechter's list. 


\section{Cheirostylis BLUME}

Cheirostylis Raymundi SCHLTr. in ENGL. 1. c. 52 (1914) 6, 56 (1921) 452.

Palau, Korror, (Raymund). Distrib. Endemic.

\section{Chilochista LINDLEY}

Chilochista Loheri Schltr. in ENGL. 1. c. 56 (1921) 491.

On a coral island, near Korror, Palau (RAYmund). Angaur (LoHer). Distrib. Endemic.

\section{Coelogyne LINDLEY}

Coelogyne guamensis Amls in Philip. Journ. Sci. 9 (1914) Bot 11; Merr. 1. c. 71 ; Schltr. 1. c. 457.

Palau Korror 110. The type from Guam. Distrit. Endemic.

Coelogyne sp. SCHLTR. 1. c. 457.

Guam (Fritz).

\section{Corymbis BLume}

Corymbis Ledermannii Schltr. in ENGL. 1. c. 56 (1921) 456.

Palau Korrsr (LedermanN); Guam (Fritz). Palau Aimiriik 261, 1949. Distrib. Endemic.

Corysanthes R. BROWN

Corysanthes ponapensis HoSOKAWA et FukUYAMA in Trans. Nat. Hist. Soc. Formos. 25 (1935) 17.

Ponape Hosokawa 5699! Distrib. Endemic.

\section{Dendrobium SWARTZ}

Dendrobium Amesianum Schltr. in ENGL. 1. c. 56 (1921) 568. Ponape: Patapat, Nanpomal (Ledermann). Distrib. Endemic.

Dendrobium brachyanthum SCHLTR. 1. c. 469.

The type from Babeldaob, Palau. Palau, Korror 185, 230, 377. Distrib. Endemic.

Dendrobium carolineuse SCHLTR. 1. c. 472.

The type from Ponape, Patapat and Nanpomal. No. 324 collectcd at Aimiriik, Palau is closely allied to this species. Distrib. Endemic. 
Dendrobium flavicole SCHLTR. 1. c. 466.

The type from Patapat, Ponape. Distrib. Endemic.

Dendrobium guamense AmFs in Philip. Journ. Sci. 9 (1914) Bot. 14; MERr. 1. c. 71 ; Schltr. 1. c. 473.

The type from Guam. Distrib. Known only from Guam.

Dendrobium Kerstingianum ScIILTR. in ENgl. 1. c. 56 (1921) 467. The type from Ngasur, Babeldaob, Palau (Ledermann). Distrib. Endemic.

Dendrobium Kraemeri SCHLTR. in ENGL. 1. c. 52 (1914) 10, 56 (1221) 471. The Type from Palau. Distrib. Endemic.

Dendrobium palawense SCHItr. 1. c. 52 (1914) 10, 56 (1921) 470. The type from Korror, Palau. Korror 231, 1891, 1989. Distrib. Endemic.

Dendrobium ponapense SCHLTR. in ENGL. I. c. 56 (1221) 471.

The type from Ponape. Niinoani-zan 813. Distrib. Endemic.

Dendrobium scopa Lindi. Bot. Reg. (1842) Misc. 55 ; Ames in Philip. Journ. Sci. 9 (1914) Bot. 13; MerR. 1. c. 71; Schltr. 1. c. 56 (1921) 469.

Desmotrichum scopa KRÄNzLIN in ENGL. Pflanzenr. IV, 50. II, 21 (1910) 349.

Guam. Distrib. Philippines, Celebes, New Guinea and Samoa.

Dendrobium violaceo-miniatum Scilırk. in ENGL. l. c. 56 (1921) 470.

The type from Patapat, Ponape at $200-300 \mathrm{~m}$ alt. (Ledermann). Distrib. Endemic.

\section{Didymoplexis GRIFFITH}

Didymoplexis fimbricata ScHLTR. in ENGL. 1. c. 56 (1921) 449.

The type from Babeldaob, Palau (Lliderman's). Distrib. Endemic.

Eulophia R. Brown

Eulophia emarginata BL. Orch. F1. Jav. (1858) 152 ; SCH.TR. 1. c. 490. Eulophia guamensis Ames in Philip. Journ. Sci. 9 (1914) Bot. 12; MrRr. 1. c. 70.

The type from Guam. Distrib. Endemic. 
Eulophia MacGregorii Ames 1. c. 12 ; MerR. 1. c. 70.

The type from Guam. Distrib. Endemic. No. 522 (Palau, Kamset) seems to be conspecific with the present species.

\section{Galeola LouREIRo}

Galeola sp. Schltr. in ENGL. 1. c. 52 (1914) 6, 56 (1921) 446. Ponape (SCHNEE).

\section{Habenaria WILLDENOW}

Habenaria carolinensis SchIITR. in ENGL. 1. c. 56 (1921) 445.

The type from Ponape, Monte Santo at $700 \mathrm{~m}$ alt. (Ledermann). Ponape, Niinoanizan 841. Distrib. Endemic.

Habenaria sp. Volkess 1. c. 461; Schltr. in ENGL. 1. c. 52 (1914) 5,56 (1921) 445.

Yap (VOLKENS).

\section{Hataeria BLUME}

Hataeria Raymundi Schltr. in Engl. 1. c. 56 (1921) 453.

Hataeria sp. SchtTr. in ENGL. 1. c. 52 (1914) 7.

The type from Korror, Palau (RAYMund). Distrib. Endemic.

Hataeria sp.

Ponape 1551. In primary forests.

\section{Liparis RICHARD}

Liparis guamensis AMES in Philip. Journ. Sci. 9 (1914) Bot. 11; MerR. 1. c. 70 ; Schitr. in FNGL. 1. c. 56 (1921) 464.

The type from Guam. Palau Aimiriik 2336. No. 518, collected at Aimiyon, Palau may also belong to this species. Distrib. Endemic.

\section{Luisia Gauidichiadd}

Luisia teretifolia Gaud. in FreYc. Voy. Bot. (1825) 427, t. 37 ; Merr. 1. c. 70 ; Schltr. in Engl. 1. c. 52 (1914) 12; 56 (1921) 494.

Guam. Distrib. The type from Guam. Widely distributed in Malaya and Polynesia. 


\section{Mediocalcar J. J. SMITH .}

Mediocalcar ponapense SchlTr. in ENGL. 1. c. 56 (1921) 475.

The type from Ponape, Patapat and Monte Santo (Ledermann). Distrib. Endemic.

\section{Microstylis NUTTALL}

Microstylis calcarea Schltr. in ENGL. 1. c. 56 (1921) 460.

The type from Korror, Palau (LedermanN). Distrib. Endemic.

Microstylis Kerstingiana SchlTR. 1. c. 460.

The type from Babeldaob, Palau (Ledermann). Distrib. Endemic.

Microstylis palawensis Schitr. 1. c. 459.

Microstylis sp. SciltTr. in EnGL. 1. c. 52 (1914) 8.

The type from Babeldaob, Palau (Ledermann). Distrib. Endemic.

Microstylis setipes ScHLTR. 1. c. 461.

The types from Babeldaob (LEDERMAnN) and Korror (RAYMund) Palau. Distrib. Endemic.

Microstylis Volkensii Sclu.Tr. 1. c. 458.

Microstylis Wallichii (non Lindo.) Vol.kens 1. c. 461.

Microstylis sp. SchlTr. in ENGL. 1. c. 52 (1914) 8.

The type from Yap (Volkens) and Babeldaob, Palau (Ledermann).

Distrib. Endemic.

\section{Mourenhoutia BLume,}

Mourenhoutia laxa Sciltr. in ENgl. 1. c. 56 (1921) 451.

The Type from Babeldaob, Palau (Ledermann). Distrib. Endemic.

Mourenhoutia laxa var. glabrata SCHLTR. 1. c. 451.

The type from Ponape Iatapat (Lmonfrmann). Distrib. Endemic.

Mourenhoutia leucantha SciLTR. 1. c. 450.

The type from Monte Santo, Ponape (Ledermann). Ponape, Niinoanizan 812. Distrib. Endemic.

\section{Nervilia Commerson ex Gaudichaud}

Nervilia Aragoana GAUdich. in Freyc. Bot. Voy. (1826) 422, t. 35 ;

MFrR. 1. c. 70 ; Scilltr. in Engl. l. c. 56 (1921) 448.

The type from Guam. Distrib. Malaya to Samoa. 
Nervilia? ovata Gaddich. in Freyc. 1. c. 422 ; SCHLTR. in ENGL. 1. c. 52 (1914) 6.

The type from Rota, Mariane Islands. Collected by Gaiddichaud but not ever since appeared. Distrib. Endemic.

Nervilia palawensis SchITR. in Engl. 1. c. 56 (1921) 447, f. 1. The type from Babeldaob, Palau (Ledermann). Yap 1127. Probably a synonym of Nervilia Aragona Gaud. Distrib. Endemic.

Oberonia LINDLEY

Oberonia palawensis Schlitr. in Engr. 1. c. 56 (1921) 462.

The type from Babeldaob, Palau (Ledermann). Distrib. Endemic.

Oberonia sp. nov. aff. 0. podostachys ScIILTR. in ENGL. 1. c. 463. The type from Babeldaob, Palau (LeDermanN).

\section{Phajus Loureiro}

Phajus amboinensis Bt. Mus. Lugd.-Bat. 2 (1856) 180; Schltr. in ENGL. 1. c. 56 (1921) 479.

Ponape, Patapat \& Paue (LedermanN). Ponape, Niinoani-zan 814. Distrib. Ambon.

\section{Phreatia LINDLEY}

Phreatia carolinensis SchITR. in ENgL. 1. c. 56 (1921) 486.

The type from Tol, Truk Islands (not Ponape) at $400-600 \mathrm{~m}$ altitudes (Ledermann). Distrib. Endemic.

Phreatia ponapensis SCIITR. in ENGL. 1. c. 487.

The type in Monte Santo, Ponape at 600-800 alt. (Ledermann). Distrib. Endemic.

Phreatia Thompsonii Ames in Philip, Journ. Sci. 9 (1914) Bot. 15; Merr. 1. c. 70; Schltr. 1. c. 486.

The type from Guam. Also from Tol, Truk and Nanpomal and Nankiop, Ponape (both Ledermann). Distrib. Endemic.

\section{Pseuderia ScIILfenter}

Pseuderia micronesiaca Schltr. in ENgl. 1. c. 56 (1921) 473.

The type from Patapat, Ponape, at $700-800 \mathrm{~m}$ alt. (TedermanN). The genus known only from Moluccas and Papua. Distrib. Endemic. 


\section{Rhyncophreatia SCHIFCHTER}

Rhyncophreatia palawensis SCHITR. in ENGL. I. c. 56 (1921) 488. The type from Korror. Palau: Korror 141, Aimirrik 283, 1948, Korror 277. Distrib. Endemic. The genus known only from New Guinea.

Rhyncophreatia nov. sp.?

Kusai 1373, 1441. In primary forests.

\section{Robiquetia Gamichaud}

Robiquetia lutea (VOLKENS) SCHLTR. in ENGL. 1. c. 52 (1914) 12, 56 (1921) 497.

Saccolobium luteum Volkens in ENGL. 1. c. 31 (1901) 461.

Yap (Volkens), Truk (KR̈̈MER). Yap 1125, 1192; Truk 1714. Distrib. Endemic.

\section{Saccolobium BLume,}

Saccolobium guamense AMEs in Philip. Journ. Sci. 9 (1914) Bot. 15; Merr. 1. c. 71; SCHLTR. in ENGL. 1. c. 56 (1921) 497.

Guam (Thompson). Distrib. Endemic.

\section{Sarcanthus LINDLEY}

Sarcanthus sp. nov. SchLTR. in ENGL. 1. c. 56 (1921) 498.

Babeldaob, Palau (Ledermann). Distrib.

\section{Sarchochilus R. BRown}

Sarchochilus sp. ScHI.TR. in ENGI. I. c. 52 (1914) 12.

Korror, Palau (Raymund). Distrib.

Sarchochilus sp.

Troas, Truk Island 614. In forests along the seashore.

\section{Spathoglottis BLUME}

Spathoglottis carolinensis Schrtr. in ENGL. 1. c. 52 (1314) 8, 56 (1921) 480.

Spathoglottis plicata (non Bi.) Volkens 1. c. 461.

Yap (Volkins), Palau, Korror (RAYmund). Yap 1159. Terrestrial in open grass land. Distrib. Endemic.

Spathoglottis micronesiaca SCHLTR. in ENGL. 1. c. 52 (1914) 9, 56 (1921) 480. 
Spathoglottis tomentosa (non LindL.) VoLKens 1. c. 461.

The types from Yap (Volkens) and Korror, Palau (RAYmund). Palau: Marikyoku 2045, Korror 111, 152, Arumonogui 2085, Aimiriik 329, 1974, 2353; Yap 1210, 1183 ; Rota 1771. Distrib. Endemic. The latter species is distinguished from the former by its smaller leaves and flowers.

Taeniophyllum Buume

Taeniophyllum mariannense SCHITR. in ENGL. 1. c. 52 (1914) 13, 56 (1921) 500.

Vanilla fasciola GAUD. in Bot. FreYc. Voy. (1826) 427.

Taeniophyllum fasciola (non REICHB. f.) SAFFORD in Contr. U.S. Nat. Herb. 9 (1905) 381.

Taeniophyllum sp. aff. T. obtusum Be.? AMES in Philip. Journ. Sci. 9 (1914) Bot. 16; Merr. 1. c. 71.

The type from Guam (Gaudichaud). Distrib. Endemic.

Taeniophyllum palawense ScHITR. in ENGL. 1. c. 56 (1911) 499, f. 3. Palau, Korror 461. Distrib. Endemic.

Taeniophyllum petrophilum ScHITR. in ENGL. I. c. 500.

The types from Mutok, Ponape; Ngarsul, Palau (Ledfirmann). Distrib. Endemic.

\section{Trichoglottis BLumE}

Trichoglottis Ledermannii SCHLTR. in ENGL. 1. c. 56 (1921) 496. The type from Korror, Palau. Palau 112, 1953, 1979, 2415. Distrib. Endemic.

\section{Trixspermum LOUREIRO}

Trixspermum arachnitiforme ScHlTR. in ENGL. 1. c. 56 (1921) 493. The type from Leperei, Ponape (Ledermann). Distrib. Endemic.

\section{Vandopsis PrITzer}

Vandopsis Raymundi SchlTR. in ENGL. 1. c. 52 (1914) 12.

Vandopsis Warocqueana (non Schltr.) SchlTr. in ENGL. 1. c. 52 (1914) 12.

The types from Korror and Babeldaob, Palau. Palau: Aimiriik 320, Marikyoku 427, a coral island 1988, 2413, Aimiriik 1920, 1983. An epiphyte on a mangrove tree. Distrib. Endemic. 
Vandopsis sp. nov.

Truk 1715. In primary forests.

\section{Vanilla SWARTZ}

Vanilla ponapensis Kanehira et Yamamoto in Trans. Nat. Hist. Formos. 23 (1933) 21.

Ponape, Sankakuyama 773 . In thickets at $100-200 \mathrm{~m}$ altitudes. An epiphyte on a dead tree. The genus new to the flora of Micronesia.

\section{Vrydagzynea BLUME}

Vrydagzynea micronesiaca SCHLTR. in ENGrL. l. c. 56 (1921) 455.

The itype from Monte Santo, Ponape at $700 \mathrm{~m}$ alt. (LedermanN). Distrib. Endemic.

\section{Zeuxine LiNdLEY}

Zeuxine Fritzii Schitr. in ENGi. 1. c. 56 (1921) 453.

The types from Ngarsul, Babeldaob, Palau (REDkrmanN) and also from Saipan (FriTz). Distrib. Endemic.

\section{Casuarinaceae}

\section{Casuarina Linnaeus}

Casuarina equisetifolia LINN. Amoen. Acad. 4 (1759) 143; KANEHIRA in Bot. Mag. Tokyo 45 (1931) 275.

Palau Angaur 574, Saipan 1013. Along the seashore. Distrib. Tropics of the Old World, pantropic in cultivation. Nat. name: "goago" (Rota), “ngasu” (Palau).

\section{Piperaceae}

Peperomia Ruzz et Pavon

Peperomia breviramula C. DC. in ENGI. Bot. Jahrb. 56 (1921) 503.

The type from Monte Santo, Ponape (Ledermann). Ponape 756. Distrib. Endemic.

Peperomia guamana C. DC. in Philip. Journ. Sci. (1914) Bot. 72.

The type from Guam. Distrib. Endemic.

Peperomia Hoeferi C. DC. in ENGL. 1. c. 505. 
The type from Saipan (Hör巨a). In coral rock. Distrib. Endemic. Nat. name: "potpot" (Saipan).

Peperomia Kraemeri C. DC. in ENGL. 1. c. 503.

Palau, Korror 243. The type from Korror, Palau (Kralimer). Distrib. Endemic.

Peperomia palauensis C. DC. in ENGL. 1. c. 505.

The type from Korror, Palau. On a coral rock (Ledermann). Distrib. Endemic.

Peperomia pellucida (LinN.) HBK. Nov. Gen. Sp. Pl. 1 (1815) 64; MerR. 1. c. 72 .

Piper pellucida Linn. Sp. Pl. (1753) 30.

Piper bilineata Miq. in Nov. Act.Acad. Nat. Cur. 19, Suppl. 1 (1843) 485; VolkeNs 1. c. 461.

Guam (Merrill), Yap (Volkrens). Distrtb. A pantropic species of American origin.

Peperomia ponapensis C. DC. in ENGL. 1. c. 504.

The type from Metaranium, Ponape. On basalt rock of the Nammatal ruin (Ledermann). Ponape 856. Distrib. Endemic.

Peperomia saipana C. DC. 1. c. 72 ; ENGL. 1. c. 56 (1921) 505.

Saipan 986, Rota 1739, Saligan 2171. The type from Saipan. Nat. name: "Popudpod" (Saipan). Distrib. Endemic.

Peperomia Volkensii C. DC. in ENGI.. 1. c. 503.

The type from Lele, Kusai (VolkENs) and also known from Boston Island, Marshall Islands (FINSCII). Distrib. Endemic.

Peperomia yapensis C. DC. 1. c. 505 .

The type from Yap (Volkens). Distrib. Endemic.

\section{Piper Linnaeus}

Piper Betle Linn. Sp. P1. (1753) 28; Volkens 1. c. 461; MerR. 1. c. 72 .

Peperomia kuscuiensis HIssikswa in Trans. Nat. Hist. Soc. Formos. 25 (1935) 120 should be compared with P. Volkensii C. DC.; Peperomia pasifica Hanks and $P$. mariannensis Hisokaws 1.c. 119-120, with Peperomia guamana C. DC. and P. saipana C. DC. respectively. 
Palau 453-, Saipan 892 ; Saligan 2161. Cultivated in Saipan, Yap, and Palau for chewing. Distrib. India to Malaya.

Piper carolinense HosoKawa in Trans. Nat. Hist. Soc. Formos. 25 (1935) 117.

Palau Hosokawa 7505! Distrib. Endemic.

Piper fragile BenTh. in Hook. Lond. Journ. Bot. 2 (1843) 234; K. Schum. et Hol.r. Fl. Kais. Wilhemsl. (1889) 36.

Palau, Korror 341. Distrib. New Guinea.

Piper guhamense C. DC. Prodr. 16, 1 (1869) 336 ; Merk. 1. c. 71; IIOSOKAWA 1. c. 117.

Guam. Distrib. Endemic, unless identical with the Polynesian Piper methysticum (MekRILL 1. c.)

Piper kusaiense Hosokawa 1. c. 118.

Kusai, Hosokawa 6324! Distrib. Endemic.

Piper majusculum Blume in Verh. Betav. Gen. 11 (1926) 210.

Palau: Marikyoku 392, Angaur 568, Tôdai-yama 2027. In thickets at low altitudes. Distrib. Malaya.

Piper methysticum FoRST. f. Fl. Escul Insular. Ocean. austr, (Diss.) (1786) 76; GuILlaum. in Journ. Arnold Arb. 13 (1932) 82.

Ponape: Kolonia 671, Niinoani-zan 835 . Usually cultivated but sometimes wild in forests. Nat. names: "shakau" (l'onape). The root used for drinking. Distrib. Polynesia.

Piper palauense Hosokawa 1. c. 118.

Palau, Babeldaob. HosoKaWa 7361. Jistrib. Endemic.

Piper ponapense C. DC. in ENGL. 1. c. 56 (1921) 502.

Ponape, Kolonia 672, 721. In primary forests at low altitudes. Distrib. Endemic.

Piper potamogetonifolium OpIZ in PresL, Rel. Haenk. 1 (1828) 156 ; MERR. 1. c. 72.

The type collected in Guam by HaEnKe. "Known only from that island, unless the plant was erroneously localized, as many HAENkE's were" (MERRILL).

Piper spp. Truk 1288, 1716; Kusai 1350, 1388; Palau 196, 286 ; Rota $1741,1753,1788$. Indeterminable specimens ; 


\section{Ulmaceae}

\section{Celtis Linnaeus}

Celtis paniculata Planch. in Ann. Sc. Nat. Sér. 3, 10 (1848) 305; KaneHIR in Bot. Mag. Tokyo 46 (1931) 275, Fl. Micr. (1933) 82 , f. 14.

Palau, a coral island near Korror 353, 2001 Yap 1181, $1253 . \quad$ In primary forests at low altitudes. Distrib. Polynesia to Australia. Nat. name: "zahle" (yap).

\section{Gironniera Gaudichaud}

Gironniera celtidifolia Gaudich. Bot. FreYC. Voy. (1844) t 85; Kanehira 1. c. 275, Fl. Micr. (1933) 83, f. 15.

Palau, Babeldaob 260, 1955, 2362 ; Ponape 797, 1608, 1626, 1651, 1654. In primary forests from low to medium altitudes. Distrib. Bismarck Archipelago, Solomon Islands, Fiji, Samoa and the Philippines. Nat. name: "Kehmut" (Ponape).

\section{Trema LOUREIRO}

Trema amboinensis (WrldD.) Blume Mus. Bot. Lugd.-Bat. 2 (1856) 61 ; KanehiRa 1. c. 275 , Fl. Micr. 85.

Celtis amboinensis Willd. Sp. Pl. 4, 2 (1805) 997.

Trema timorensis Blume 1. c. 62; VolkENs 1. c. 461.

Palau: Korror 155, 2003, Babeldaob 530, 2346; Yap 1098. In thickets and secondary forests. Distrib. Southern China to the Malay Peninsula, Philippines, southward to New Guinea and northeastern Australia.

Trema orientalis BL. var. argentea (PL.) LAUTERB. in ENGL. 1. c. 50 (2913) 320, f. B ; Kanehira in Bot. Mag. Tokyo 45 (1931) 275, Fl. Micr. 85.

Tinian 52, Saipan 925, Anatahan 2143, Pagan 2206, Palau 1973, 2338. In thickets and secondary forests at low altitudes. Distrib. India to Polynesia (the species), Bonin and Marianne Islands (the variety). Nat. name: "banahl" (Saipan).

\section{Moraceae}

\section{Artocarpus FoRSTER}

Artocarpus communis Forst. Char. Gen. (1776) 101; KANEHIRA 
in Bot. Mag. Tokyo 45 (1931) 276, Fl. Micr. (1933) 86, f. 16. Ponape 726; Palau Angaur 571; Saipan 897. Distrib. A native tree, widely cultivated. Nat. names: Seedless variety: "mohs" (Kusai), "lemme" (Saipan), "lemai" (Rota), "mai" (Ponape), " arudu," "muzuh" (Palau), "sou" (Yap), " neoda" (Truk). Seeded varicty: " mai chait" (Ponape), "rokurok" (Saipan), "mei chon" (edible), "aroyas" (not edible) (Truk).

Artocarpus integra (Thunb.) MerR. Interpret. Herb. Amb. (1917) $190 ;$ KANeHiRA 1. c. 276, Fl. Micr. 87.

Radermachia integra Thuns. in Vet. Akad. Handl. Stockh. (1776) 254.

Yap 1090. Planted also in Ponape, Palau, etc. Distrib. A native of tropical Asia, now widely distributed in cultivation.

\section{Ficus Linnaeus}

Ficus carolinensis Warb. in Schum.-LauTerb. Fl. Deutsch. Schutzg. Südsee (1905) 242; Kanehira in Bot. Mag. Tokyo 45 (1931) 276, Fl. Micr. (1933) 77, f. 17.

Ficus Saffordii Merr. 1. c. 74 .

The type from Marianne Islands (HAENKE's collection) and FrTTZ's specimen from Saipan. Tinian 49; Yap 69, 70; Ponape 749, 771; Saipan 912, 997. Guam (MerRILL). In primary forests at low altitudes. Distrib. Endemic. (Marianne and Caroline Islands).

Ficus mariannensis MerR. in Philip. Journ. Sci. 9 (1914) Bot. 73. The type from Guam. Distrib. Endemic. Nat. name: "nunu" (Guam).

Ficus philippinensis MiQ. Hook. Journ. Lond. Bot. 7 (1848) 435; KANEHIRA 1. c. 276, Fl. Micr. 88.

Ponape, Kolonia $648,710,1537$. In thickets and forests at low altitudes. Distrib. Celebes, Moluccas, New Caledonia and the Philippines. Nat. names: "neen" (Ponape).

Ficus ramentacea RoxB. Fl. Ind. ed. 2, 3 (1832) 216; KANEIIIRA I. c. 276 , Fl. Micr. 89 , f. 18.

Palau: Korror 184, 337, Babeldaob 379, 429, Aimiriik 1981, 2306. In thickets and secondary forests, an epiphyte climber. Distrib. Burma to southern China through Malaya to the Palau Islands. 
Ficus retusa Linn. Mant. 1 (1767) 129; KaneiriRa 1. c. 276, Fl. Micr. 90.

Palau: Korror 143, Armatin 475, Angaur 573, a coral island 1907, 2009; Yap 1120; Kusai, Moto 1430. In thickets at low altitudes. Distrib. India to southern China, Formosa through Malaya to Australia and New Caledonia. Nat. Name: "nunu" (Saipan).

Ficus Senfftiana Warb. in Schum.-LaUtrerb. 1. c. 247; Kanehira 1. c. 276, Fl. Micr. 90, f. 19.

The type from Yap (VoLKLNS). Palau, Korror 157, 1852, Babeldaob 310; Yap 1091. In forests along the sea shore. Distrib. Endemic.

Fieus tenuistipula Merr. in Philip. Journ. Sci. 9 (1914) Bot. 75; KaneHIRA in Bot. Mag. Tokyo 49 (1935) 271.

Saipan 2252. The type from Guam. Distrib. Endemic.

Ficus tinctoria Forst. f. Prodr. (1786) 76 ; KaneinRA 1. c. 276, Fl. Micr. 91, f. 20.

Saipan 1, 15, 919, 943, 2228 ; Palau, Korror 158, Angaur 576; Tinian 65, 913, 1073; Yap 1171, 1201; Aramagan 2192; Rota 1765; Truk 1271. In thickets and secondary forests. Distrib. Philippines to Polynesia.

\section{Malaisia BLANCO}

Malaisia scandens (Lour.) Pranch. in Ann. Sci. Nat. Bot. 4, 3 (1855) 293 ; Kanehira in Bot. Mag. Tokyo 45 (1931) 22\%, Fl. Micr. (1933) 92.

Caturus scandens Lour. Fl. Cochinch. (1790) 613.

Saipan 917, 931; Rota 1778. In thickets and edge of forests from low to medium altitudes.

Distrib. China and Formosa to Malaya, Australia and Polynesia.

\section{Morus LinNaEus}

Morus alba Linn. Sp. Pl. (1753) 986; Merr. 1. c. 73.

Guam. Distrib. A native of subtemperate Asia, now cultivated in all warm countries.

\section{Urticaceae}

\section{Boehmeria JACQUIN}

Boehmeria macrophylla D. DoN, Prodr. Fl. Nep. (1825) 60, var. 
Ponape, Kolonia 717; Aramagan 2185. In the edge of primary forests at low altitude. Distrib. Himalaya (the species).

Boehmeria nivea (LINN.) Gaudich. Freyc. Bot. Voy. (1826) 499; MERR. 1. c. 76.

Urtica nivea Linn. Sp. Pl. (1753) 895.

Guam. Distrib. A native of tropical Asia, now widely distributed in cultivation.

Boehmeria platyphylla Don, Prodr. F1. Nepal. (1825) 60.

Kusai 1331, 1332. In thickets at low altitudes. Distrib. India to Africa, China, Japan, Malaya and tropical Australia.

\section{Cypholophus WedDell}

Cypholophus moluccanus (BL.) MiQ. Ann. Mus. Lugd.-Bat. 4 (1869) 305.

Urtrica moluccana B1. Bijdr. (1825) 491.

Ponape: Niinoani-zan 834, Tortom-zan 1552, Nânaraut-zan 1645 . In primary forests at about $500-600 \mathrm{~m}$ alt. Distrib. Sumatra, Philippines, Formosa (Botel Tobago) to Hawaii.

\section{Elatostema FORSTER}

Elatostema calcareum MERR. 1. c. 77.

Rota 1734. The type from Guam. In wet jungles. Distrib. Endemic.

Elatostema kusaiense KANEHIRA in Trans. Nat. Hist. Soc. Formos. 25 (1935) 1, f. 1.

Kusai 1330, 1407!. In dense wet primary forests at about $100 \mathrm{~m}$ altitude. Distrib. Endemic.

Elatostema stenophyllum MERR. 1. c. 76.

The type from Guam. Distrib. Endemic.

Elatostema stolouiforme KANEHIRA 1. c. 2, f, 2.

Palau 311. In wet jungles. Distrib. Endemic.

Fleurya Gaudichadd

Fleurya interrupta (LinN.) GAUDICH. Freyc. Bot. Voy. (1826) 497 ;

VOLKENS 1. c. 462 ; MerR. 1. c. 78.

Urtica interrupta Linn. Sp. Pl. (1753) 985.

Yap (Volkens), Guam (Merrill). Ponape, Mctaranium 789; along the road. Distrib. Abyssinia to Australia and Polynesia. 
Fleurya ruderalis Gaudich. 1. c. 497 ; VotKens 1. c. 462; Merr. 1. c. 78 ; KoIDz. in Bot. Mag. Tokyo 29 (1915) 248.

Palau: Angaur 581, on coral rock, Korror 228, 1872; Yap 1214; Kusai 1306. Along the seashore. Distrib. Philippines, Java to New Guinea and Polynesia.

\section{Laportea GAUDICHAUD}

Laportea kusaiana KanehIRA in Bot. Mag. Tokyo 46 (1932) 449, Fl. Micr. (1933) 93, f. 21.

Kusai 1304! 1324! Ponape 3561. In forests from low to medium altitudes. Distrib. Endemic. The type from Kusai. Nat. name: "Lelesi" (Ponape).

Laportea saipanensis KanehIRA in Bot. Mag. Tokyo 45 (1931) 277, Fl. Micr. (1933) 94, f. 22.

Saipan 5, 887, 973! 2218, 2251; Rota 1754, 1776, 1783. In primary and secondary forests. Distrib. Endemic. Nat. names: "kahtat" (Saipan), " kahtl" (Rota).

\section{Pilea LINDLEY}

Pilea microphylla (LinN.) LieBM. in Vidensk. Selsk. Skr. 5, 2 (1851) 302 ; KoIDz. in Bot. Mag. Tokyo 29 (1915) 248.

Parielaria microphylla Linn. Syst. ed. 10 (1759) 1308.

Palau, Korror 109, 2029; Ponape 875; Kusai 1300. In damp places near houses. Jaluit (KolDzumi). Distrib. A native of tropical America, now in most tropical countries.

Oreocnide Miquel

Oreocnide sp.

Ponape, Sankaku-yama 762. In forests at low altitudes. A scandent shrub.

Oreocnide? sp.

Palau, in a coral island 250. A small shrub.

\section{Pipturus WEDDELL}

Pipturus argenteus (FoRST. f.) WedD. in DC. Prodr. 16, 1 (1869) 235; Kanehira in Bot. Mag. Tokyo 45 (1931) 277, Fl. Micr. (1933) 96. 
Saipan 32, 47, 898, 937, 979; Saligan 2172; Rota 1772; Kusai 1302. In primary forests at medium alt. Distrib. Malay Archipelago to Polynesia. Nat. name: "amahazan" (Rota).

Pipturus incanus WedD. 1. c. 235; KoIDz. in Bot. Mag. Tokyo 29 (1915) 248.

Jauit (KoIDzumt). Distrib. Malaya to Polynesia.

Piptutus micronesicus KanehIRA in Bot. Mag. Tokyo 49 (1935) 353.

Palau 250, 1870, 2433! In forests at low altitudes. Distrib. Endemic.

Pipturus repandus (Blume) WedD. in Arch Mus. Paris 9 (1856) 448 ; Merr. Enum. Philip. Fl. Pl. 2 (1923) 95.

Urtica repanda BL. Bijdr. (1825) 501.

Ponape 723, 762, 1487, 1695; Truk 1293. In forest at low altitudes. Distrib. Sumatra, Philippines to Moluccas. A scandent shrub.

\section{Pouzolzia Gaudichald}

Pouzolzia zeylanica (LINN.) BENN. PI. Jav. Rar. (1838, 67.

Parietaria zeylanica LinN. Sp. Pl. (1753) 131.

Pouzolzia indica Gatd. Bot. Freyc. Voy. (1826) 503; Volkens 1. c. 462.

Palau Kamset 526, 2097. Along a road at low altitudes. Yap (VoLKENS). Jistrib. A polymorphous species in Indo-Malayan distribution.

\section{Procris COMMERSON}

Procris pedunculata (FoRst. f.) WeDD. in DC. Prodr. 14, 1 (1869) 191; Merr. 1. c. 78.

Elatostema pedunculatum ForsT. f. Prodr. (1776) 105, t. 53.

Palau, Korror 253, 375, 2005; Ponape, Kolonia 734, Sankakuyama 758, Parkier 734, Kity 1534; Saipan 595; Kusai 1401; Guam. In damp primary forests at low altitudes. Distrib. Malay Archipelago to Polynesia.

\section{Proteaceae}

Helicia Lourfiro

Helicia micronesica KANEHIRA Fl. Micr. (1933) 95, f. 23, et in Bot. Mag. Tokyo 47 (1933) 669. 
Palau, Aimiriik 1970 ! 2113, 2337. In thickets and forests at low altitudes. Nistrib. Endemic.

\section{Santalaceae}

\section{Santalum Linnaeus}

Santalum album LiNN. Sp. Pl. (1753) 849.

Ponape, Kolonia 1583. Planted in the Experiment Station. Distrib. India. Sandalwood tree.

\section{Olacaceae \\ Olax Linnaeus}

Olax imbricata Roxв. Hort. Beng. (1814) 5, nom. nud., F1. Ind. 1 (1820) 169 ; KANEHRA in Bot. Mag. Tokyo 45 (1931) 277.

Palau, Korror 76, 2783. In thickets along the seashore. Disirib. India, Philippines to Malaya.

\section{Ximenia Plumier}

Ximenia americana LiNN. Sp. Pl. (1753) 1193; KANẼIIRA in Bot. Mag. Tokyo 45 (1931) 278, Fl. Micr. (1933) 96.

Tinian 59, 1045, 1065; Palau Angaur 596; Kusai 1376; Rota 1813. In thickets immediately back of the beach along the seashore. A small spiny three or shrub. 1)istrib. A pantropic strand plant.

\section{Loranthaceae}

\section{Loranthus LiNNAEUS}

Loranthus caudatifolius KANEHIRA in Bot. Mag. Tokyo 46 (1932) 450, Fl. Micr. (1933) 98, f. 24.

Ponape Nânaraut-zan 1665! Metaranium 803. In forests at $400-700 \mathrm{~m}$ altitudes. Distrib. Endemic.

Loranthus ponapensis KANEHIRA 1. c. 450, Fl. Micr. 98, f. 25.

Ponape 1637 ! In primary forests, Nânarautzan at $550 \mathrm{~m}$ alt. Distrib. Endemic.

\section{Balanophoraceae}

\section{Balaniella PH, van TIEgheM}

Balaniella pedicellaris (Schltr.) Hosokawa in Trans. Nat. Hist, Soc. 
Formos. 24 (1934) 201, Journ. Soc. Trop. Agr. 6 (1934) 572. Balanophora pedicellaris SCHLTR. in ENGL. Bot. Jahrb. 52 (1915) 14, f. 1 .

The type from Truk, Mount. Tol (KräMER). Truk Tol, 1298. In primary forests at $300-450 \mathrm{~m}$ altitudes. 1)istrib. Endemic.

\section{Balanophora FoRsTER}

Balanophora mariannae Hosokawa in Journ. Trop. Agr. 6 (1934) 572.

The type from Saligan, it is also known from Saipan and Aramagan (fide Hosokawa). Distrib. Endemic.

Balanophora pentamera VAN TIEGH. in Ann. Sci. Nat. 9, 6 (1907) 151; MerR. 1. c. $78 .^{*}$

The type from Guam. Distrib. Endemic.

\section{Aristolochiaceae}

\section{Aristolochia LinNAEUS}

Aristolochia elegans MAST. in Gard. Chron. 2, 24 (1885) 301; Merr. I. c. 79.

Guam. Distrib. A native of tropical America, now cultivated in many tropical countries.

\section{Polygonaceae}

\section{Antigonon ENDLICHFR}

Antigonon leptus Hook. et ArN. Bot. Beech. Voy. (1841) 308, t. 69. Ponape, Saipan. Cultivated. Distrib. A native of tropical America, now widely distributed in cultivation.

\section{Polygonum Linnaeus}

Polygonum barbatum LinN. Sp. Pl. (1753) 362 ; Merr. 1. c. 80. Guam. In open wet places. Jistrib. Tropical Asia to Malaya.

$\because$ "This species was very briefiy characterized by VAN THEGIEM, but his description is very inalequate. The type was from the Marianne Islands, collected by MAkcus: in the year 1889. I at first considered our Guam material to represent a distinct species, as Van Thoua describes Balmophora pentamera as having usually 5-merous flowers, but sometimes with 6 or even 7 sepals, which does not agree with our specimens. The probubilities are, however, that a single species is represented, and while letling my description stand, I have adopted Vav Twimms specific name." (Mi:RRILl 1. C.) 


\section{Chenopodiaceae}

\section{Chenopodium Linnaeus}

Chenopodium album Lins. Sp. P1. (1753) 219 ; Merr. I. c. 80.

Guam. Distrib. Widely distributed in the temperate and tropical regions of both hemispheres, probably originally European. Jap. name: Akaza.

Chenopodium ambrosioides Linn. Sp. P1. (1753) 219 ; MEkr. 1. c. 80. Guam. isisirib. A native of Mexico, now widely distributed in the warmer parts of both hemispheres. Jap. name: Arita-sô.

\section{Amaranthaceae \\ Achyranthes LinNaEus}

Achyranthes aspera Linv. Sp. Pl. (1753) 204; Merr. 1. c. 80.

Guam. A weed in open grass places. Distrib. Pantropic, probably originating in the eastern hemisphere.

Achyranthes bidentata BLume Bijdr. (1825) 545 .

Palau Angaur 580, Saligan 2169. In old clearings. Distrib. India to Philippines and Java.

\section{Alternanthera FORSKẢL}

Alternanthera denticulata R. BR. Prod. 1, 417; Volkens 1. c. 462. A weed in wet places. Yap (Volkens). Wistrib. Pantropic.

Alternanthera sessilis (Linn.) R. Bk. ex R. \& S. Syst. 5 (1819) 554 ; VOLKENS 1. c. 554.

Gomphrena sessilis LiNN. Sp. Pl. ed. 2 (1762) 300.

Yap (Volkens). Jistrib. Pantropic. Jap. name: Turu-nogeitô.

Alternanthera versicolor REGEL in Gartenflora (1869) 101; MERR. 1. c. 81 .

Guam. Cultivated. 1)istrib. Apparently a native of tropical America.

\section{Amaranthus LinNaEus}

Amaranthus Blitum Linn. Sp. Pl. (1753) 990 ; KoIDz. in Bot. Mag. Tokyo 29 (IS15) 248. Jaluit (KoIDzumi). Distrib. Temperate and tropical zones.

Amaranthus spinosus LinN. Sp. Pl. (1753) 991; Merr. 1. c. 80. 
Palau Angaur 679; Saipan 962; Anatahan 2147. A weed in open waste places. Distrib. Pantropic. Jap. name: Haribiyu.

Amaranthus tricolor LiNn. Sp. Pl. (1753) 989; Merr. 1. c. 80.

Guam. Commonly cultivated, occasionally a weed. I)istrib. Pantropic. Jap. name: Seiyô-hageitô.

Amaranthus viridis Linv. Sp. Pl. ed. 2 (1763) 1405 ; Volkens 1. c. 462 ; Merr. 1. c. 81.

Yap and Guam. Probably introduced. Distrib. Pantropic.

Amaranthus sp.

Kusai 1409. In thickets.

\section{Celosia Linnaeus}

Celosia cristata Linn. Sp. Pl. (1753) 205 ; MERr. 1. c. 81.

Guam. Introduced. Distrib. A native of tropical America.

Cyathula Loureiro

Cyathula prostrata (Linn.) Budme Bijdr. (1825) 549; Volkens 1. c. 462.

Achyranthes prostrata LinN. Sp. Pl. ed. 2 (1762) 296.

Ponape 852. In waste places. Distrib. Pantropic. Jap. name: Inokodutimodoki.

Deeringia R. Brown

Deeringia amaranthoides (LAM.) MERr. Interpret. Herb. Amb. (1917) 211 ; KankHIRA in Bot. Mag. Tokyo 45 (1931) 278.

Achyranthes amaranthoides LaM. Encycl. 1 (1785) 548.

Saipan 40,983 . In the edge of primary forests at medium altitudes. 1)istrib. India to China, Formosa and Philippines southward to Australia. Nat. name: "pehzerak" (Saipan).

\section{Gomphrena Linnaeus}

Gomphrena globosa LinN. Sp. Pl. (1753) 224; Merr. 1. c. 81.

Guam; Kusai 1466. Distrib. $\Lambda$ native of tropical countries, now cultivated or naturalized in all warm countries. Jap. name: Senniti-sô.

\section{Psilotrichum LinNAEuS}

Psilotrichum trichotomum Blume Bijdr. (1825) 545. 
Palau, Kamset 527. In open waste places. IIstrib. India to the Malay Peninsula, Sumatra, Java, Philippines and Borneo.

\section{Nyctaginaceae}

\section{Boerhaavia LINNEus}

Boerhaavia diffusa Linn. Sp. Pl. (175̃3) 3; Merk. I. c. 81. Saipan 1034, 1041; Rota 1818. A common weed in and about towns. Distrib. Pantropic.

\section{Bougainvillaea COMMERSON}

Bougainvillaea spectabilis WIIJ.). Sp. PI. 2 (1799) 348.

Saipan, Ponape etc. Planted in gardens. Distrib. A native of Madagascar, now widely cultivated for ornamental purposes. Jap. name: Ikadakazura.

\section{Mirabilis LinNAEUS}

Mirabilis jalapa Linn. Sp. Pl. (1753) 177; Merk. 1. c. 81; KoIdz. in Bot. Mag. Tokyo 29 (1915) 249.

Guam; Jaluit; Kusai 1417. Distrib. A native of Mexico, now cultivated in all warm countries. Jap. name: Osiroi-bana.

\section{Pisonia Linnaeus}

Pisonia grandis R. Br. Prodr. (1810) 422; MFrr. 1. c. 82 ; KANEHIRA in Bot. Mag. Tokyo 45 (1931) 278, Fl. Mir. (1933) 98.

Pisonia Brunnoniana ENDL. Prodr. Fl. Norf. (1833) 43; Volkens 1. c. 462 .

Saipan 4, 968, 2219, 2245, 2256, Rota 1809 . In primary forests at low and medium altitudes. The tree is very abundant in Tinian. /'istrib. Philippines, Moluccas, north-eastern Australia across Polynesia to the Palmyra Islands. Nat.names: "umum" (Saipan), " umomo" (Rota).

Pisonia umbellifera (Forst.) SELM. in Bonplandia 10 (1862) 154; Kanehilka 1. c. 278, Fi. Micr. 100, f. 26.

Ceodes umbellifera Forst. Char. Gen. (1776) 71, t. 71.

Saipan 969, 570, 585, 2235; Palau: Tôdai-yama 1858, Peliliu 2425, Angaur 578,1846 . In thickets and primary forests. /istrib. Formosa, Philippines, Java and Malaya to Australia and Poly- 
nesia. Nat. name: "langsat" (Saipan). Jap. name: Nagamino-udonoki.

\section{Aizoaceae \\ Mollugo Linnaeus}

Mollugo pentaphylla LiNN. Sp. P1. (1753) 89; MFrR. 1. c. 82.

Palau, Korror 372; Yap 1262. A weed in cultivated areas. Distrib. India to Japan southward to Malaya. Jap. name: Zakurosô.

\section{Sesuvium Linnaeus}

Sesuvium portulacastrum LinN. Syst. cd. 10 (1759) 1058; MERR. 1. c. 82 .

Guam. Along sandy seashores. Distrib. Pantropic. Jap. name: Hama-mizuna.

\section{Portulacaceae}

Portulaca Linnaeus

Portulaca quadrifida LINN. Mant. 1 (1767) 73; VolKens I. c. 462. MerR. 1. c. 82.

Palau Marikyoku 454, Armatin 479; Saipan 1012. A weed in and about villages. Distrib. Tropical Asia and Africa to Malaya.

Portulaca oleracea LiNN. Sp. Pl. (1753) 445; Volkens 1. c. 462, Merr. 1. c. 82 ; KoIDz. in Bot. Mag. Tokyo 29 (1915) 249.

Yap, Guam, Jaluit. A weed. Distrib. All warm countries. Jap. name: Tonbo-gusa.

\section{Ceratophyllaceae \\ Ceratophyllum Linnaeus}

Ceratophyllum demersum LiNN. Sp. Pl. (1753) 992 ; MErR. 1. c. 82. A weed in fresh water. Guam. Distrib. All warm countries. Jap. name: Kingyo-mo.

\section{Menispermaceae \\ Pachygone MIERS}

Pachygone Ledermanni Difls in ENGI. Bot. Jahrb. 56 (1921) 507; KANEHIRA in Bot. Mag. 45 (1931) 278. 
Palau, Kamset 541. The type from Babeldaob, Palau. A climber in forests at low altitudes. Distrib. Endemic.

Pachygone ponapensis KaneHIRA Mss.

Ponape, Kolonia 669, 1519.

\section{Tinospora MIERS}

Tinospora homosepala DIELS in Philip. Journ. Sci. 8 (1913) Bot. 158 ; MERR. 1. c. 83.

The type from Guam. Distrib. Endemic.

\section{Anonaceae}

\section{Anona Linnaeus}

Anona glabra LinN. Sp. Pl. (1753) 537; KANEIIIRA in Bot. Mag. Tokyo 45 (1931) 278.

Ponape 872. Planted. Distrib. Introduced from tropical America.

Anona muricata Linn. Sp. Pl. (1753) 537; KANeHiRa 1. c. 278.

Palau Aimiyon 507; Ponape, Metaranium 782 ; Saipan 2250. Planted. D)istrib. A native of tropical America, now widely in cultivation. Nat. name: "syasyap" (Saipan).

Anona reticulata Linn. Sp. Pl. (1753) 537; KANEHIRA 1. c. 279.

Saipan 44, Tinian 1058. Planted. Distrib. Introduced from tropical America, now pantropic in cultivation.

Anona squamosa Linn. Sp. Pl. (1753) 537; Merr. 1. c. 83; KaneHIRA Fl. Micr. (1933) 103.

Guam, Saipan. Distrib. A native of tropical America, now cultivated in all tropical countries.

\section{Canangium BaILlON}

Canangium odoratum (LAm.) BaIL. ex KING in Journ. As. Soc. Beng. 4I, 2 (1892) 41; KANEHIRA 1. c. 279.

Uvaria odorata LAM. Encycl. 1 (1785) 595.

Ponape, Kolonia 870. Planted in gardens, road sides, sometimes in thickets in and about towns. Distrib. Indigenous in Burma and Java. Planted in most tropical countries.

Goniothalamus Hook. f. et THOMson

Goniothalamus carolinensis KANEHIRA in Bot. Mag. Tokyo 45 (1931) 279, Fl. Micr. (1933) 104, f. 28. 
Palau Armatin 477, Kamset 531, New Gaspan 2125. Distrib. Endemic.

\section{Guamia MerriLL}

Guamia mariannae (SAFFord) Merr. in Journ. Sci. 10 (1915) Bot. 243; KanehiRa 1. c. 45 (1931) 279, Fl. Micr. (1933) 106, f. 29. Papualthia mariannae SAFFoRD in Journ. Washingt. Acad. Sci. 2 (1912) 456; Dieis in ENGL. Bot. Jahrb. 52 (1914) 16, f. 2.

Polyalthia mariannae Mrrk. in Philip. Journ. Sci. 9 (1914) Bot. 83, Saipan 14, 883, 2236; Tinian 56, 1050, 1074, 2259; Rota 1805, 1768; Pagan 2199. Very common in forests and thickets from low to about $200 \mathrm{~m}$ alt. Distrib. The type from Guam, endemic. Nat. name: "paipai."

\section{Polyalthia BLume}

Polyalthia Merrillii KanehiRa Fl. Micr. (1933) 108, f. 30 seminudum in Bot. Mag. Tokyo 47 (1933) 669.

Palau Tôdai-yama 2025. In dense primary forests at $100 \mathrm{~m}$ altitudes. 1)istrib. Endemic.

\section{Myristicaceae \\ Horsfieldia WiLLDENOW}

Horsfieldia amklaal KankHIRA Fl. Micr. (1833) 109, f. 31, Bot. Mag. Bot. Mag. Tokyo 47 (1933) 669.

Palau Babeldaob 564, 1944, 1978, 2058! 2059! In primary forests at low altitudes. Distrib. Endemic. Nat. name: "amklaal" (Palau).

Horsfieldia nunu KaneHIRA in Bot. Mag. Tokyo 46 (1932) 451, Fl. Micr. 111, f. 32 .

Kusai 1303 ! 1314! In primary forests at medium altitudes. 1)istrib. Endemic. Nat. name: "nunu" (Kusai).

Horsfieldia palauensis KanEHIRA 1. c. 452, Fl. Micr. 111, f. 32. Palau': Aimiriik 270! 1847, 1958! 1560, 2371 ; Korror 1847, Gaspan 482, Peliliu 2440. In primary forests. 1)istrib. Endemic.

\section{Myristica LinnaEus}

Myristica hypargyraea A. GRAY in Bot. U. S. Expl. Exped. (1854) 
33 ; Kanehtra in Bot. Mag. Tokyo 45 (1931) 280, Fl. Micr. (1933) 113 , f. 34.

Ponape: Parkier 727, Kolonia 763, Metaranium 788, Kity 1512, 1529, 1545. In primary forests at low altitudes. Distrib. The type from Navigator (Samoan) Islands. Nat. name: "karala" (Ponape).

Myristica insularis KaneHIRA semi-nud., Fl. Micr. 115. f. 35, Bot. Mag. Tokyo 47 (1933) 671.

Palau Tôdai-yama 1865 ! In dense primary forests at $150 \mathrm{~m}$ altitude. Distrib. Endemic.

\section{Lauraceae}

\section{Cassytha Linnaeus}

Cassytha filiformis LinN. Sp. Pl. (1753) 35; Volkenss 1. c. 462; MerR. 1. c. 84; Kordz. in Bot. Mag. Tokyo 29 1915) 249; KaneHIRA in Bot. Mag. Tokyo 45 (1931) 280.

Palau Korror 371; Saipan 1043. A strand climber. Distrib. Pantropic- Jap. name: Suna-zuru.

\section{Cinnamomum TOURNEFORT}

Cinnamomum carolinense KorDz. in Bot. Mag. Tokyo 30 (1916) 401 ; Kanehira 1. c. 280, Fl. Micr. (1533) 117, f. 36.

Cinnamomum carolinense var. oblongum KANEHIRA in Bot. Mag. Tokyo 46 (1932) 452, Fl. Micr. 118, f. 37.

Ponape, Kolonia 639, 1492, 1524, 2139; Kusai Moto 1434, 1351. In thickets and primary forests about $100 \mathrm{~m}$ altitudes. Distrib. The type from Ponape. Endemic. Nat. name: "mattieu" (Ponape). The bark boiled in water is used for drinking.

Cinnamomum sessilifolium KanehrRa 1. c. 453, Fl. Micr. 119, f. 38. Ponape Nânaraut-zan 1658! In forests at about $700 \mathrm{~m}$ altitude. Distrib. Endemic.

\section{Persea Plumifr}

Persea americana Mis.. Gard. Dict. ed. 8 (1768).

Persea gratissima Gaektw. Fruct. 3 (1805-07) 222. 
Ponape 1588. Planted in the garden of the Experiment Station. Distrib. A native of tropical America.

\section{Henandiaceae}

\section{Hernandia Linnaeus}

Hernandia ovigera LINN. in Stickm. Herb, Amb. 1754) 14, Amoen. Acad. 4 (1757) 125; Mersn. in DC. Prodr. 15, 1 (1864) 262 ; Kanehira in Bot. Mag. Tokyo 45 (1931) 280. F1. Micr. (1933) 121 , f. 39.

Hernandia peltata MEISN. 1. c. 263 ; MERR. 1. c. 249.

Palau Marikyoku 435; Saipan 888; Kusai 1413; Rota 1780. Distrib. Ceylon to tropical East Africa, Madagascar, through Malaya to tropical Australia and Polyncsia. Nat. names: "dokoo" (Palau), "gachal" (Yap), "nônak" (Saipan and Rota), "punpun" (Kusai).

\section{Capparidaceae}

\section{Capparis Linnaeus}

Capparis carolinensis KANEHIRA in Bot. Mag. Tokyo 48 (1934) 919. 1. 6.

Palau, Peliliu 2428. In thicket at low altitudes. Distrib. Endemic.

Capparis cordifolia LaM. Encycl. 1, (1785) 609; MERR. 1. c. 84; Kanehira in Bot. Mag. Tokyo 45 (1931) 280.

Capparis mariana JACQ. Hort. Schoenbr. 1 (1797) 109.

Tinian 1070, 10522, 2277; Saipan 1020; Rota 1812; Palau, a coral island 1994. Along the seashore. Distrib. Endemic.

\section{Crataeva Linnaeus}

Crataeva speciosa VolKens 1. c. 463; KANEHIRA in Bot. Mag. Tokyo 45 (1931) 281.

Palau Korror 80, 2067, 2765; Truk 603; Yap 1106; Truk 1285; Ponape 1548. Very common in Yap; planted or growing spontaneously in Truk, Ponape and Palau. Distrib. Endemic. Nat. names; " abeech" (Yap), "kapopsung" (Palau), " abûts" (Truk). 


\section{Gynandropsis de Candolle}

Gynandropsis speciosa (HBK.) DC. Prodr. 1 (1824) 238.

Cleome speciosa HBK. Nov. Gen. Sp. Pl. 5 (1817) 84, t. 436.

Ponape 853. In waste places near houses. Distrib. A native of tropcal America, now pantropic.

\section{Polanisia RAFINESQUE}

Polanisia icosandra (LINN.) W. et A. Prodr. (1834) 22.

Cleome icosandra LINN. Sp. P1. (1753) 672.

Cleome viscosa LinN. 1. c. ; MERR. 1. c. 85.

Saipan 909. A common weed in the settled area. Distrib. Pantropic. Jap. name: Hime-fûtyôso.

\section{Cruciferae}

\section{Brassica TOURNEFORT}

Brassica integrifolia (WEST) O. E. SCHULZ var. timoriana (DC.) O. E. Schulz in ENGL. Bot. Jahrb. 56 (1921) 507.

Sinapis integrifolia West Bijdr. St. Croix (1793) 296.

Ponape, Metaranium, Atol Napali (LedermanN). On sandy shores Distrib. The species pantropic.

Brassica juncea (Linn.) Coss. in Bull. Soc. Bot. Fr. 6 (1859) 609; MERR. 1. c. 84.

Sinapis juncea LinN. Sp. Pl. (1753) 668.

Guam. Distrib. A native of Asia, now widely distributed in all warm countries wild or cultivated. Jap. name: Takana.

\section{Raphanus LinNaEus}

Raphanus sativus Linn. Sp. Pl. (1753) 669; Merr. 1. c. 84.

Distrib. Cultivated in all warm countries. Jap. name: Hatukadaikon.

\section{Moringaceae}

Moringa BuRMAN

Moringa oleifera. LAM. Encycl. 1 (1785) 398. 
Morinaga pterygiosperma. GaERTN. Fruct. 2 (1791) 314.

Ponape 1680. Planted in the Experiment Station. Distrib. Probably a native of India, now cultivated in all tropical countries.

\section{Nepenthaceae}

Nepenthes LinNaEus

Nepenthes mirabilis (LoUR.) Merr. Interpret. Herb. Amb. (1917) 242; KANEHIRA in Bot. Mag. 45 (1931) 231.

Phyllamphora mirabilis Louk. Fl. Cochinch. (1790) 606.

Nepenthes phyllamphora WILld. Sp. Pl. 4, 2 (1805) 874; Volkens 1. c. 463.

Palau Korror 78, Aimiriik 283, Marikyoku 420, 2051; Yap 1117. Very common in open fields and waste places in Palau and Yap. Distrib. Southern China and Indo-China, through Malaya and the Philippines to New Guinea. Nat. names: "finnabolo" or " mariek" (Yap), " amireek" (Palau).

\section{Droseraceae}

\section{Drosera LINNAEUS}

Drosera Burmanni VAHI. Symb. 3 (1794) 50; DIEIS in ENGL. Pflanzenr. 26 (1906) 75, t. 27, E-G.

Palau 2047. In open places. Distrib. India to Japan, Philippines southward to northern Australia. Jap. name: Kurumabamôsengoke.

\section{Crassulaceae}

\section{Bryophyllum SALISBURY}

Bryophyllum pinnatum (LAM.) KuRz in Journ. As. Soc. Beng. 40, 2 (1876) 309; MERR. 1. c. 85.

Cotyledon pinnatum LAM. Encycl. 2 (1786) 171.

Bryophyllum calycinum SALISB. Parad. Lond. (1805) t. 3 ; KoIDz. in Bot. Mag. Tokyo 29 (1915) 249.

Jaluit (KoIDzumi). Distrib. In all tropical countries, probably a native of the eastern hemisphere. Jap. name: Seiron-benkei. 


\section{Pittosporum BANKS}

Pittosporum ponapense KANEHIRA in Bot. Mag. Tokyo 45 (1931) 281, Fl. Micr. (1933) 124, f. 40.

Ponape Kolonia 659 ! 1470! Truk 1292, 1273, 1718. In the edge of primary forests. Distrib. Endemic. Nat. name: "kamal" (Ponape).

Pittosporum kusaiense KanehiRa 1. c. 46 (1932) 454, Fl. Micr. 125 , f. 41.

Kusai 1336 ! 1375 ! In primary forests at $400-500 \mathrm{~m}$ altitudes. Nat. name: "cane" (Kusai).

\section{Rosaceae}

\section{Eriobotyra LINDLEY}

Eriobotrya japonica LiNDL. in Trans.. Linn. Soc. 13 (1822) 102.

Ponape Kolonia 1600. Planted. Iistrib. Japan to China. Jap. name: Biwa.

\section{Parinarium Aublet}

Parinarium corymbosum (Butme) MrQ., Fl. Ind. Bat. 1, 1 (1855) 356.

Maranthes corymbosa BL. Bijdr. (1825) 162.

Palau 166. In thickets. Distrib. India through Malaya, Philippines to tropical Australia.

Parinarium glaberrimum Hassk. in Tijdschr. Nat. Gesch. 10 (1843) 147, nom. nud., Cat. Hort. Bogor. (1844) 269, nom. nud., Fl. 27 (1844) 583; Kanehira in Bot. Mag. Tokyo 45 (1931) 282, Fl. Micr. 127, f. 42.

Parinarium laurinum A. Gray Bot. Wilkes U. S. Explor. Exped. (1854) 490 , t. 55.

Palau: Aimiriik 2296, Marikyoku 433, Babeldaob 2818, Arakabesan 2818; Ponape 847, 1538; Yap 1213; Kusai 1337. In forests near houses, sometimes in primary forests from low to medium altitudes; very abundant in Kusai. Distrib. Java, Borneo Philippines to Polynesia. Nat. names: "ais" (Ponape, Truk), "gritin" or "agaritin" (Palau, Yap), "yarado" or "adidi" 
(Yap), "aset" (Kusai). Seeds oily and used for painting canoes and houses.

Parinarium palauense KANEHIRA in Bot. Mag. Tokyo 45 (1931) 282, Fl. Micr. 128, f. 43.

Palau Babeldaob Armatin 469!, Aimiriik 1940, 2373, 2753, Ogiwal 2060. In primary forests. Distrib. Endemic. Nat. name: “apgau” (Palau).

\section{Rubus TOURNEFORT}

Rubus moluccanus LiNN. Sp. Pl. (1753) 1197.

Kusai 1338, 1394. In forests at medium altitudes. Distrib. India, through Malaya and Philippines to Australia and Fiji. Nat. name: "kôkor" (Kusai).

\section{Connaraceae \\ Connarus Linnafus}

Connarus Gaudichaudii (DC.) PIANCH. in Linnaea 23 (1850) 429; Scheldenb. in Engl. Bot. Jahrb. 59 (1924) 17; Kanehira in Bot. Mag. Tokyo 45 (1931) 282, Fl. Micr. (1933) 129, f. 44.

Omphalobium Gaudichaudii DC. Prodr. 2 (1825) 85.

Palau: Korror 81, 144, 364, 1894, 2074, 2392, 2464, Aimion 514, Arumonogui 2090, Aimiriik 1919, 2355. In thickets at low altitudes. Distrib. Moluccas to New Guinea.

\section{Leguminosae}

\section{Abrus Linnaeus}

Abrus precatorius LinN. Syst. ed. 12 (1767) 472; Volkens 1. c. 464; Merre. 1. c. 91 ; KanehiRa in Bot. Mag. Tokyo 45 (1931) 283.

Ponape Metaranium 857; Tinian 1083. Very common in thickets and waste places. Distrib. Pantropic. Jap. name: Tôazuki.

\section{Acacia Willdenow}

Acacia confusa MerR. in Philip. Journ. Sci. 5 (1910) Bot. 27.

Planted in Saipan, Palau, Ponape. Distrib. Philippines, southern China to Formosa. Introduced. Jap. name: Sôsijyu. 
Acacia Farnesiana (Linn.) Willd. Sp. Pl. 4, 2 (1805) 1083 ; Merr. 1. c. 86 .

Mimosa Farnesiana LiNn. Sp. Pl. (1753) 521.

Guam. Distrib. A native of tropical America, now in all warm countries.

Jap. name: Gingôkan.

Acacia laurifolia WiLld. Sp. Pl. 4 (1805) 1053.

Rota 3532. In thickets. Distrib. Ranging from Fiji and Samoa to the New Hebrides and New Caledonia. A species new to the Micronesian flora. The specimen identified by Dr. Merkill.

\section{Adenanthera RoYEN}

Adenanthera pavonina LiNv. Sp. Pl. (1753) 385; MerR. 1. c. 86. Guam. Kusai 1335. In forests at medium altitudes. Distrib. A native of tropical Asia, now widely distributed in cultivation. Nat. name: "metekam" (Kusai).

\section{Aeschynomene Linnaeus}

Aeschynomene indica LINN. Sp. PI. (1753) 713; MERR. 1. c. 90, Guam. Distrib. Widely distributed in the tropics of the Old World. Jap. name: Kusanemu.

\section{Albizzia DurazzinI}

Albizzia acle (Blanco) Merr. in Philip. Journ. Sci. 5 (1910) Bot. 25. Mimosa acle BI.ANCO Fl. Filip. (1837) 738.

Ponape Kolonia 667. Planted in avenues. Distrib. Introduced from Philippines.

Albizzia Lebbeck (LINN.) BENTH. in Hook. Lond. Journ. Bot. 3 (1844) 87; KaneHIRA in Bot. Mag. Tokyo 45 (1931) 283.

Mimosa Lebbeck LiNN. Sp. Pl. (1753) 516.

Saipan 1084; Yap 1256. Planted in gardens. Distrib. A native of tropical Africa, now widely distributed in cultivation. Jap. name: Biruma-nemu.

Albizzia retusa Benth. in Hook. 1. c. 3 (1844) 90 ; VolKens 1. c. 463.

Yap 1256. Distrib. Nicobar Islands across Malaya, Philippines to Polynesia. 


\section{Alysciearpus Necker}

Alyscicarpus nummularifolium (LinN.) DC. Prodr. 2 (1825) 353; MerR. 1. c. 91.

Hedysarum nummularifolium LiNN. Sp. Pl. (1753) 746.

Guam. Distrib. Widely distributed in the Indo-Malayan rigion, introduced in tropical America. Jap. name: Nagaba-takehagi.

Alyscicarpus vaginalis (LinN.) DC. Prodr. 2 (1825) 353 ; Volkens 1. c. 464.

Hedysarum vaginale LinN. Sp. Pl. (1753) 746.

Yap 1261. In open grassland. Distrib. Tropical Asia and Malaya.

\section{Arachis LinnaEus}

Arachis hypogaea LiNN. Sp. Pl. (1753) 741 ; Merr. 1. c. 90.

Cultivated. Distrib. $\Lambda$ native of tropical America, now widely distributed in all warm countries. Jap. name: Nankin-mame.

\section{Bauhinia Linnaeus}

Bauhinia monandra KURz in Journ. As. Soc. Beng. 42, 2 (1873) 73; MERR. 1. c. 87.

Ponape, Kolonia 874. Planted for ornaments. /istrib. A native of tropical America, now pantropic.

Bauhinia tomentosa LinN. Sp. Pl. (1753) 375; Merk. 1. c. 87.

Guam. Distrib. A native of India, now widely distributed in cultivation.

\section{Caesalpinia Linnaeus}

Caesalpinia crista LinN. Sp. Pl. (1753) 380; Merr. 1. c. 88.

Guam. Distrib. Pantropic. Jap. name: Sirotubu.

Caesalpinia jayabo MAZA in Anal. Soc. Esp. Hist. Nat. 19 (1890) 234 ; KANEHIRA in Bot. Mag. Tokyo 45 (1931) 283.

Caesalpinia glabra Merr. 1. c. 88, non Guilandina glabra MiLl.

Palau Aimiriik 330; Rota 1808. Distrib. Pantropic. Jap. name: Hasunomi-kadura.

Caesalpinia nuga (LInn.) Ait. Hort. Kew. ed. 2, 3 (1811) 32; VOLKENS 1. c. 464 ; KANEHIRA 1. c. 283.

Guilandina nuga LinN. Sp. Pl. (1753) 546. 
Palau, Korror 360 2010, 2780; Yap 1207. On coral rock near the sea. Distrib. Pantropic. Jap. name: Nanten-kudura.

Caesalpinia pulcherrima (Linn.) Sw. Obs. (1791) 166; Volkens 1. c. 464 ; MERR. l. c. 89.

Poinciana pulcherrima LiNN. Sp. Pl. (1753) 380.

Ponape, Kolonia 1678, 1679. Planted. in gardens. Distrib. Origin of tropical America, now pantropic.

Caesalpinia Sappan LINN. Sp. Pl. (1753) 381; MERr. 1. c. 89.

Guam. Distrib. Widely distributed in the Indo-Malayan region and in other tropical countries. Jap. name: Suwô-no-ki.

\section{Cajanus de Candolse}

Cajanus cajan (Mill.) Millsp. in Field Columb. Mus. Bot. 2 (1900) 53; MERR. 1. c. 94.

Guam. Distrib. Probably a native of tropical Asia, now cultivated in all tropical countries. Jap. name: Kimame.

\section{Canavalia de Candolle}

Canavalia ensiformis (Linn.) DC. Prodr. 2 (1825) 404; VolKens 1. c. 464 ; Merr. 1. c. 93; KoIDz. in Bot. Mag. Tokyo 29 (1915) 250.

Dolichos ensiformis LiNn. Sp. Pl. (1753) 725.

Guam, Yap, Jaluit. Iistrib. A native of the West Indies.

Canavalia lineata (Thuns.) DC. Prodr. 2 (1825) 404 ; Merr. 1. c. 92. Dolichos lineata ThunB. Fl. Jap. (1784) 280.

Guam. Distrib. Pantropic, along sandy seashores. Jap. name: Hama-natamame.

Canavalia megalantha Merr. in Philip. Journ. Sci. 9 (1914) Bot. 93. The type from Guam. Distrib. Endemic.

Canavalia microcarpa (DC.) PIPER in Proc. Biol. Soc. Wash. 30 (1917) 177.

Lablab microcarpa DC. Prodr. 2 (1825) 402.

Canavalia turgida Grah. in Wall. Cat. (1832) No. 5j34; Merr. 1. c. 92 .

Commonly near the seashore. Distrib. India to Malaya, southern China and Formosa to Polynesia. Jap. name: Takanatamame. 
Cantharospermum WigHT et ARNOTT

Cantharospermum scarabaeoides (LinN.) BAIL. Bull. Soc. Linn. Paris 1 (1883) 384 ; Merk. 1. c. 93.

Dolichos scarabaeoides LinN. Sp. P1. (1753) 726.

Guam; Saipan 966. In open grasslands. 1)istrib. India to the Mascarene Islands, southern China to Malaya. Jap. name: Birôdohimeuzu.

\section{Cassia LinNaeus}

Cassia alata LINN. Sp. PI. (1753) 378; Merr. 1. c. 88.

Palau, Armatin 481; Ponape, Metaranium 783. Distrib. Introduced from tropical America, now pantropic. Jap. name: Hane-senna.

Cassia fistula LINN. Sp. Pl. (1753) 377; MERk. l. c. 88.

Ponape, Kolonia 1586. Introduced recently by Dr. T. KARIYoNE. Distrib. A native of tropical Asia, now widely distributed. Jap. name: Nanban-saikati.

Cassia mimosoides LrnN. Sp. Pl. (1753) 379; Merr. 1. c. 88.

Guam; Palau (Okamoto); Pagan 2202. Distrib. India to Japan southward to Australia.

Cassia occidentalis LinN. Sp. Pl. (1753) 377; Volkens 1. c. 563; Merr. 1. c. 88; KoIDz. in Bot. Mag. Tokyo 29 (1915) 250.

Saipan 959; Palau, Korror 177, Angaur 583; Jaluit; Yap. Distrib. A native of tropical America, now pantropic. Jap. name: Habusô.

Cassia Sophora Linn. Sp. Pl. (1753) 379; Volkens 1. c. 463 ; Merr. 1. c. 88 .

Guam, Yap. Distrib. The same distribution as the preceeding.

Cassia surattensis Burm. f. Fl. Ind. (1768) 97.

Kusai 1460. Cultivated in garden. Distrib. A native of tropical America, now widespread in cultivation as a shade tree.

Cassia Tora Linn. Sp. P1. (1753) 376; Volkens 1. c. 463; Merr. 1. c. 88 .

Guam, Yap, Palau (OKamoTo). Distrib. Same as the two preceeding. Jap. name: Ebisu-gusa, Habutya.

Clitoria Linnaeus

Clitoria ternata LINN. (1753) 753 ; MERR. 1. c. 92. 
Guam. Iistrib. Probably a native of tropical America, now pantropic. Jap. name: Tyômame.

\section{Crotalaria Dillenius}

Crotalaria linifolia Linn. f. Suppl. (1781) 322; VolkeNs 1. c. 464. Yap (Volkens). Distrib. India to southern China and Formosa southward through Malaya to Australia. Jap. name: Hosobatanukimame.

Crotalaria quinquefolia LinN. Sp. Pl. (1753) 716; Mrkr. 1. c. 89. Guam. Distrib. Pantropic, probably a native of tropical America. Jap. name: Kibana-hagi.

Crotalaria trifoliastrum WILLD. Sp. Pl. 3 (1800) 983; HosoKAWA in Trans. Nat. Hist. Soc. Formos. 25 (1935) 18.

Pagan Hosokawa 7974 ! Distrib. India, Malaya, Philippines to Australia.

\section{Cynometra Linnafus}

Cynometra carolinensis KanfiriRA in Pot. Mag. Tokyo 56 (1932) 455, Fl. Micr. 133, f. 45.

The type from Palau 510! (not 5101); Marikyoku 431, Korror 2764, 2785, Aimiriik 314, 1982; Saipan 8, S15, 979.

Cynometra carolinensis var. glabrescens KANEHIRA

Palau Aimiriik 1915! (flos.), Marikyoku 2754 (flos.), Babeldaob 384 (sterile) ; Yap 1166 (fr.)

Cynometra Hosinoi Kanehira Mss.

Cynometra ramiflora (non LinN.) KaneHIRA in Bot. Mag. Tokyo 45 (i931) 283. The species is distinguished from $C$. carolinensis by its glabrous inflorescences, much larger leaves and nearly globose fruit which is rounded at the tip.

Ponape Kolonia 2140! (flos.) 662 (fr.), 1525 (fr.), 1543 (fr.). In thickets and forests.

Cynometra Yokotai Kanemrika 1. c. 454, Fl. Micr. 134, f. 46.

The type from Truk, Tol, 1277! In forests at medium altitudes. Distrib. Endemic.

\section{Dalbergia Linnaeus $f$.}

Dalbergia candenatensis (Dennst.) Prain in Journ. As. Soc. Beng. 
72, 2 (1901) 49; Merr. 1. c. 91; Kanehira in Bot. Mag. Tokyo 45 (1931) 283.

Cassia candenatensis Dennst. Schlüs. Hort. Malabar. (1818) 32.

Dalbergia torta GRAH. VoLkENS 1. c. 464.

I’alau Korror 194, Ponape Kolonia 670; Yap 1208. In thickets along the seashore. Distrib. India to southern China southward to New Guinea and Australia. A strictly littoral species.

Dalbergia ferruginea Roxв. Hort. Beng. (1814) 98, nom. nud., Fl. Ind. ed. 2, 3 (1832) 228; VolKFNS 1. c. 464.

Yap (Volkess). Distrib. Borneo, Philippines to New Guinea.

Dalbergia oligophylla HosoKawa in Trans. Nat. Hist. Soc. Formos. 24 (1934) 415.

Palau, Babeldaob Hosokawa 7298! 7)istrit. Endemic.

Dalbergia palauensis HosoKawa 1. c. 415.

Palau Babeldaob, Hosokawa 6766 ! Jistrib. Endemic.

\section{Delonix RAFINESQUE}

Delonix regia (BoJ.) RAF. Fl. Tellur. 2 (1836) 92; MERr. 1. c. 88; KaneHIRA in Bot. Mag. Tokyo 45 (1931) 285.

Poinciana regia BoJ. in CuRTis's Bot. Mag. 56 (1829) t. 2884 ; VolKENS 1. c. 463.

Planted for ornamental purpose in Saipan, Palau, Ponape etc.

7) istrib. A native of Madagascar, now pantropic in cultivation. Jap. name: Hôwôboku.

\section{Derris LOUREIRO}

Derris elliptica (Roxb.) Benth. in Journ. Linn. Soc. Bot. 4 (1860) Suppl. 111 ; Volkens 1. c. 464; Kanfinira in Bot. Mag. Tokyo 45 (1931) 284.

Galedupa elliptica Bentr. Hort. Beng. (1814) 53.

Palau, Marikyoku 446; Ponape 675; Yap 1163. Distrib. Chittagong through Malaya to New Guinea and Bismarck Archipelago. The plant is commonly cultivated and the roots are used for poisoning fish. Nat. names: "duup" (Palau), "yubu" (Yap), " uup" (Truk), "oop" (Kusai).

Derris mariannensis Hosokawa in Trans. Nat. Hist. Soc. Formos. 25 (1935) 124. 
Rota Hosokawa 7592. Distrib. Endemic.

Derris trifoliata Lour. Fl. Cochinch. (1790) 433; KanEIIIRA 1. c. 283. Derris uliginosa BENTH. in MrQ. Pl. Jungh. (1852) 252; Volkens 1. c. 464.

Palau Korror 140, 174, 254; Marikyoku 2048; Truk 602; Ponape. Kolonia 718; Kusai 1355. A climber in littoral forests. Distrib. Tropical East Africa through Asia and Malaya to Australia.

\section{Desmodium Desvaux}

Desmodium capitatum (BurM. f.) DC. Prodr. 2 (1825) 336 ; VoLKENS 1. c. 464.

Hedysarum capitatum BuRM. f. Fl. Ind. (1768) 167, t. 64, f. 1.

Yap (VolkeNS). Iistrib. India to Malaya.

Desmodium giganticum (Linn.) DC. Prodr. 2 (1825) 327; MrRr. 1. c. 90 .

Hedysarum giganticum LINN. Sp. Pl. (1753) 746.

Guam. Jistrib. Through the tropics of the Old World, introduced in the West Indies. Jap. name: Tamatunagi.

Desmodium heterocarpum (LINN.) DC. Prodr. 2 (1825) 337 ; KANEHIRA in Bot. Mag. Tokyo 45 (1931) 284.

Hedysarum heterocarpum LinN. Sp. Pl. (1753) 747.

Desmodium polycarpum DC. Prodr. 2 (1825) 334 ; VolKens 1. c. 464. Yap 1172; Ponape 1517, 1574. In open grasslands. Distrib. Tropical Aftrica and Asia through Malaya to Australia.

Desmodium heterophyllum (WILLD.) DC. Prodr. 2 (1825) 334; VoLKFNS 1. c. 464 ; Merr. 1. c. 90.

Hedysarum heterophyllum WIILD. Sp. Pl. 3 (1800) 1201.

Yap. Distrib. India to the Mascarene Islands, southern China and Malaya.

Desmodium triflorum (LInN.) DC. Prodr. 2 (1825) 334; VolkENs 1. c. 464 ; MERr. 1. c. 91.

Hedysarum triflorum LinN. Sp. P1. (1753) 749.

Palau, Korror 139 ; Ponape Metaranium 780. Aramagan 2183.

Distrib. Pantropic. Jap. name: Hai-makiehagi.

Desmodium umbellatum (Linn.) DC. Prodr. 2 (1825) 325; VOLKENS 1. c. 464 ; Merr. 1. c. 284. 
Hedysarum umbellatum Linv. Sp. Pl. (1753) 747.

Palau, Korror 96, 161, 2409, Marikyoku 437; Ponape Kolonia 690, Parkier 1511; Saipan 1018; Rota 1820.

In forests and thickets at low altitudes or in strand jungles. /Nistrib. Mascarcne Islands and tropical Asia through Malaya to northern Australia. Jap, name: Ookihagi.

Dioclea Humboldt, Bonpland et Kunth

Dioclea reflexa Hook. f. Niger Flora (1849) 306; VolkFins 1. c. 464. Yap (Vol.Kens). Distrib. Pantropic.

\section{Dolichos Linnafus}

Dolichos Lablab LinN. Sp. Pl. (1753) 725; Volkfins I. c. 464; Merr. I. c. 94.

Guam, Yap. Distrib. Pantropic in cultivation. Jap. name: Hudjmame.

\section{Entada ADANSON}

Entada phaseoloides (LINN.) Mlerr, in Philip. Journ. Sci. 9 (1914) Bot. 86 ; Kanfinka in Bot. Mag. Tokyo 45 (1931) 284.

Lens phaseoloides LinN. in Stickm. Herb. Amb. (1754) 18.

Palau Korror 121; Ponape 750; Saipan 990; Ponape 1697. In forests at low and medium altitudes. Distrib. Pantropic. Nat. names: "bozokdonkle" (Saipan), "kesegeguh " (Yap).

\section{Erythrina LinNaEus}

Erythrina fuscus Lour. Fl. Cochinch. (1790) 427; KANEHIRA in Bot. Mag. Tokyo 45 (1931) 427.

Ponape 746,1689 . In swampy places near the sca. Distrib. India to Indo-China southward to Malaya and Polynesia.

Erythrina variegata LiNN. in Stickm. Herb. Amb. (1754) 10; KANEHIRA I. c. 284.

Erythrina indica Lam. Encycl. 2 (1785) 391; Volkins 1. c. 464; Merk. 1. c. 92 ; KoIDz. in Bot. Mag. Tokyo 29 (1915) 250.

Saipan 907, 1078. In forests near the seashore. Distrib. India, Philippines to Polynesia. Nat. names: "gau-gau" (Saipan), "ral," "Iolo," (Yap). 


\section{Flemingia ROXBURGH}

Flemingia strobifera (LINN.) R. BR. in Ait. Hort. Kew. ed. 2, 4 (1812) 350 ; KaneHIRA in Bot. Mag. Tokyo 45 (1931) 285.

Hedysarum strobifera Linn. Sp. Pl. (1753) 764.

Yap 1205. Planted. 1)istrib. India to Malaya ; introduced in Mauritius and the West Indies.

\section{Gliricidia Humboldt, BonPland et KunTh}

Gliricidia sepium (JACQ.) STFEud. nomencl. (1821) 688; VotKens 1. c. 464 .

Robinia sepium JACQ. Enum. (1760) 28.

Yap. Distrib. A native of Mexico, introduced.

\section{Glycine Linnaeus}

Glycine cladestina WendL. DC. Prodr. 2 (1825) 241 ; Benth. Fl. Austr. 2 (1864) 243; Hosokawa in Trans. Nat. Hist. Soc. Formos. 25 (1935) 18.

Tinian, Pangan and Saipan (Hosokawa). Distrib. Australia to Formosa.

\section{Indigofera LinNAEUS}

Indigofera suffruticosa Mil.. Gard. Dict. ed. 8 (1768) No. 2; MerR. I. c. 89; Degener New Ill. Fl. Hawaiian Isl. 169 c.

Guam. Jistrib. A native of tropical America, now pantropic. Jap. name: Nanban-koma-tunagi.

Indigofera tinctoria LiNN. Sp. P1. (1753) 751 ; Merr. 1. c. 90. Guam. Distrib. Pantropic. Jap. name: Taiwan-komatunagi.

\section{Inocarpus FORSTER}

Inocarpus edulis Forst. Char. Gen. (1776) 66, t. 33; Volkens 1. c. 464; Merr. 1. c. 91; Kanehira in Bot. Mag. Tokyo 45 (1931) 285, Fl. Micr. (1933) 140, f. 47.

Yap 1170; Palau Korror 344. Very common in Palau, Yap, Ponape. Kusai and Truk but not found in Saipan. In thickets and forests near houses; frequently planted; the seeds are a favorite food among the natives. Distrib. Malay Archipelago. Nat. names: "marrap" (Ponape), "keam" (Palau), "bui" (Yap), "clark" (Kusai). 


\section{Intsia THOUARS}

Intsia bijuga (Colebr.) O. Kunt7e, Rev. Gen. Pl. (1891) 192; Merr. 1. c. 87 ; KANEHIRA 1. c. 285, Fl. Micr. 142, f. 48.

Macrolobium bijugum CoLEBR. in Trans. LinN. Soc. 12 (1819) 359, t. 17.

Afzelia bijuga A. Gray Bot. Wilkes U. S. Explor. Exped. (1854) 467, t. 51 ; VOLKeNs 1. c. 463.

Palau, Korror 363, 2401; Ponape Metaranium 854, 1693, Kolonia 1575; Saipan 905; Yap 1198; Rota 1751. In primary forests at low altitudes, usually along the seashore. Distrib. Madagascar across Malaya to Polynesia. Nat. names: "show," "wantal," "zolt" (Palau and Yap); "ifil" (Saipan); "tuamis" or "kuren" (Truk). Jap. name: Siroyona.

\section{Leucaena BENTHAM}

Leucaena glauca (LinN.) BENTH. in Hook. Journ. Bot. 4 (1842) 416; Volkens 1. c. 463 ; Merr. 1. c. 86; KanehIRA in Bot. Mag. Tokyo 45 (1931) 285.

Mimosa glauca LinN. Sp. Pl. (1753) 520 .

Saipan 960. Introduced from tropical America, now pantropic. Nat. name: "tagan-tagan." Jap. name: "Gingokwan.

\section{Macropsychanthus HARMS}

Macropsychanthus carolinensis KANEHIRA et HosOKAWA in Trans. Nat. Hist. Soc. Formos. 24 (1934) 414.

Moen, Truk 1711. In forests at medium altitudes. A genus new to the flora of Micronesia.

\section{Mimosa Linnaeus}

Mimosa pudica LinN. Sp. PI. (1753) 518.

Ponape Kolonia 863. Distrib. Introduced from tropical America, now a pantropic weed. Jap. name: Nemurigusa.

\section{Medicago LinnaEus}

Medicago denticulata Willd. Sp. PI. 3 (1803) 1414; MERR. 1. c. 89. Guam. I)istrib. A native of Europe, now cultivated in the north warm countries. Jap. name: Umakoyasi. 